\title{
Abstracts of the 113th Annual Meeting of the Swiss Neurological Society, Congress Centre Kursaal Interlaken, Switzerland, November 18-19, 2021
}

\author{
Hans Jung ${ }^{1, *}$ and Urs Fischer ${ }^{2}$ \\ 1 Department of Neurology, University Hospital Zurich, 8091 Zurich, Switzerland \\ 2 Department of Neurology, University Hospital Basel and University of Basel, 4031 Basel, Switzerland; \\ Urs.Fischer@usb.ch \\ * Correspondence: hans.jung@usz.ch
}

Citation: Jung, H.; Fischer, U. Abstracts of the 113th Annual Meeting of the Swiss Neurological Society, Congress Centre Kursaal Interlaken, Switzerland, November 18-19, 2021. Clin. Transl. Neurosci. 2021, 5, 22. https://doi.org/ $10.3390 / \operatorname{ctn} 5030022$

Academic Editor: Claudio Bassetti

Received: 14 November 2021

Accepted: 14 November 2021

Published: 15 November 2021

Publisher's Note: MDPI stays neutral with regard to jurisdictional claims in published maps and institutional affiliations.

Copyright: (c) 2021 by the authors. Licensee MDPI, Basel, Switzerland. This article is an open access article distributed under the terms and conditions of the Creative Commons Attribution (CC BY) license (https:/ / creativecommons.org/licenses/by/ $4.0 /)$.

\begin{abstract}
On behalf of Swiss Neurological Society together with the Swiss Society for Behavioral Neurology, we are pleased to present the Abstracts of the 113th Annual Meeting, which was held from 18-19 November 2021. Fifteen (15) abstracts were selected for oral presentations and sixty-one (61) abstracts were selected as poster presentations. We congratulate all the presenters on their research work and contribution.
\end{abstract}

Keywords: neurology; behavioral neurology; dementia

All abstracts and information are published as submitted by the authors and are not the responsibility of the CTN journal (Clinical and Translational Neuroscience) or the meeting organizer.

\section{O01-O15 Oral Presentations}

P01-P61 Poster Presentations

O01 Interim Analysis of Autologous Hematopoietic Stem Cell Transplantation in Multiple Sclerosis (aHSCT-in-MS) in Switzerland

I. Jelcic ${ }^{1}$, A. Müller ${ }^{2}$, M. Hilty ${ }^{1}$, I. Reichen ${ }^{1}$, K. Jordan ${ }^{3}$, N. Wolfensberger ${ }^{2}$, P. Stathopoulos ${ }^{1}$, J. Ruder ${ }^{1}$, S. Ferrer ${ }^{1}$, K. Ritter ${ }^{2}$, M. Foege ${ }^{1}$, A. Lutterotti ${ }^{1}$, U. Schanz ${ }^{2}$, R. Martin ${ }^{1}$

1 Department of Neurology, University Hospital and University of Zurich, Switzerland

2 Department of Medical Oncology and Hematology, University Hospital and University of Zurich, Switzerland

3 Department of Consultation-Liaison Psychiatry and Psychosomatic Medicine, University Hospital and University of Zurich, Switzerland

Background and aim: Autologous hematopoietic stem cell transplantation (aHSCT) is used for the treatment of highly active relapsing-remitting or progressive multiple sclerosis (MS) since 1995. Based on strong data regarding efficacy and improved safety of aHSCT in MS, the Swiss Federal Office of Public Health (FOPH) granted approval in June 2018 with the requirement that patients participate in a prospective registry at our center ("aHSCT-inMS"). We here present the first safety and efficacy data for aHSCT-in-MS in Switzerland. Methods: Prospective cohort study on 35 MS patients treated with aHSCT at our center according to the BEAM-ATG protocol, because they experienced inflammatory breakthrough activity and/or progression of MS despite highly effective disease-modifying therapy (DMT). For safety analysis, adverse events (AE) of all 35 patients were examined. For efficacy analysis, outcome in 26 patients, who had $>12$ months of follow-up, was analyzed. 
Primary outcome was no evidence of disease activity (NEDA), i.e., no relapses, new or contrast-enhancing MRI lesions and clinical progression, defined as progressive worsening of expanded disability status scale (EDSS).

Results: At baseline, median age was 40 years (range 25-54), median disease duration 9.1 years (range 1.6-19.7) and median EDSS 4.8 (2.5-6.5). 15 (42.9\%) patients had relapsingremitting MS, 20 (57.1\%) progressive MS. Failure of approved DMT before aHSCT included ocrelizumab (11), rituximab (11), fingolimod (11) and natalizumab (4). Mean post-aHSCT follow-up was $17.5 \pm 12.5$ months. Most patients developed infectious AE, most frequently mucositis and/or enteritis (29/35) and usually in the first 20 days post-aHSCT. Serious AE occurred in 22/35 patients with consecutive good recovery. 2 patients with secondary progressive MS committed assisted suicide 19 and 23 months post-aHSCT because of subjective perception of progression, although NEDA was confirmed in both cases. NEDA status was achieved in 19/26 (73.1\%) patients at last follow-up. After 12 months, we observed sustained EDSS improvement in 14/26 (53.8\%) patients, stable EDSS in 6/26 (23.1\%) and EDSS-relevant progression in 6/26 (23.1\%) patients.

Conclusions: Overall, safety of aHSCT-in-MS is acceptable, and efficacy outcomes are similar to other aHSCT studies and better than approved DMT. However, aHSCT-inMS requires thorough psychiatric evaluation, vigilant monitoring (especially regarding infectious AE) and antimicrobial prophylaxis.

O02 Comparative Analysis of Dimethyl Fumarate and Teriflunomide in Relapsing-Remitting Multiple Sclerosis

\section{J. Müller ${ }^{1}$, S. Schädelin ${ }^{2}$, P. Benkert ${ }^{2}$, P. Hänni ${ }^{3}$, J. Schmid ${ }^{3}$, L. Kappos ${ }^{1}$, T. Derfuss ${ }^{1}$, J. Kuhle ${ }^{1}$, Ö. Yaldizli ${ }^{1}$}

1 Neurologic Clinic and Policlinic, MS Center and Research Center for Clinical Neuroimmunology and Neuroscience Basel (RC2NB), University Hospital Basel and University of Basel, Switzerland

2 Clinical Trial Unit, University Hospital Basel, Switzerland

3 Swiss Federation for Common Tasks of Health Insurances (SVK), Switzerland

Introduction: Dimethyl fumarate (DMF) and teriflunomide (TFL) are two oral drugs that reduce the relapse rate in patients with relapsing-remitting multiple sclerosis (RRMS). A direct comparison with a head-to-head randomized controlled trial has not been performed, yet. Analyses from observational studies comparing the effectiveness of DMF and TFL showed conflicting results.

Objective: To compare the clinical outcomes in patients on DMF and TFL in a realworld setting, where both drugs are licensed as a first-line disease modifying therapy (DMT) for RRMS.

Methods: We included all patients who initiated either DMF or TFL between January 2013 and March 2020 in the Swiss National Treatment Registry governed by the Swiss Federation for Common Tasks of Health Insurances (SVK). Coarsened exact matching was applied at timepoint of DMT initiation using age, gender, disease duration, baseline Expanded Disability Status Scale (EDSS) score, time since last relapse and relapse rate in the previous year as covariates. Time to relapse and time to 12 months confirmed EDSS worsening were analyzed using a pairwise censored Cox-proportional hazard model. Patients who switched from DMF to TFL and vice versa were assessed in mixed proportional hazard Cox models. Sensitivity analyses included the analysis of unmatched groups and inverse probability of treatment weighted data.

Results: In total, 1625 RRMS patients were included (DMF: $n=1214$; TFL: $n=411$ ); 1222 of them were matched (DMF: $n=902,72.2 \%$ female, age $44.5 \pm 10.5$ years, disease duration $8.6 \pm 7.7$ years, median EDSS 2.0; TFL: $n=320,72.4 \%$ female, age $44.4 \pm 10.5$ years, disease duration $8.7 \pm 7.6$ years, median EDSS 2.0). Time to relapse was similar between DMF and 
TFL when analyzing the unmatched groups. However, time to relapse was longer in the DMF than TFL group in the matched analysis (HR 0.72, 95\%CI 0.54-0.97, $p=0.03$ ) which was confirmed in the weighted analysis (HR $0.75,95 \%$ CI $0.58-0.98, p=0.02$ ). Time to EDSS worsening was longer in the DMF than in the TFL group (unmatched: HR $0.58,95 \% \mathrm{CI}$ $0.39-0.84, p<0.01$, with similar results in matched and weighted analyses). In patients who switched from DMF to TFL or vice versa $(n=80)$, time to relapse was longer on DMF than TFL (HR $0.38,95 \% \mathrm{CI} 0.18-0.80, p=0.01$ ), irrespective of the drug sequence.

Conclusions: Analysis of real-world data showed that in RRMS, treatment with DMF was associated with more favorable clinical outcomes compared to TFL.

O03 The Basel Version of the Awareness of Social Inference Test-Emotion Recognition (BASIT-ER): Preliminary Validation Analyses in Healthy Subjects

\author{
M. Jarsch ${ }^{1}$, K. Bartsch ${ }^{1}$, M. Berres ${ }^{2}$, I. Ryf ${ }^{3}$, K. Jurisic ${ }^{3}$, C. Sluka ${ }^{4}$, R.W. Kressig ${ }^{5}$, \\ A.U. Monsch ${ }^{3}$, O. Piguet ${ }^{6}$, M. Sollberger ${ }^{7}$ \\ 1 Memory Clinic, University Department of Geriatric Medicine FELIX PLATTER/Faculty \\ of Psychology, University of Basel, Switzerland \\ 2 Faculty of Mathematics and Technology, University of Applied Science Koblenz, Ger- \\ many \\ 3 Memory Clinic, University Department of Geriatric Medicine FELIX PLATTER, Basel, \\ Switzerland \\ 4 Department of Clinical Research, University of Basel and University Hospital Basel, \\ Switzerland \\ 5 University Department of Geriatric Medicine FELIX PLATTER, Basel, Switzerland \\ 6 School of Psychology and Brain and Mind Centre, The University of Sydney, Australia \\ 7 Memory Clinic, University Department of Geriatric Medicine FELIX PLATTER/Department \\ of Neurology, University Hospital Basel, Switzerland
}

Background and Objectives: Emotion recognition (ER) is commonly impaired in many brain disorders. Therefore, its reliable and ecologically valid examination is a crucial part of any comprehensive neuropsychological assessment. In this regard, an established English-language test identifying deficits in ER is the "The Awareness of Social Inference Test-Emotion Evaluation Test" (TASIT-EET). However, a comparable German-language ER test is currently missing. In this study, we aimed to develop the first German-language TASIT-EET adaption for use in clinical populations.

Materials and Methods: We selected 22 scenes [three scenes per emotion (i.e., anger, disgust, fear, happiness, sadness, surprise, and neutral) and one practice scene] out of the 56 TASIT-EET scenes. In collaboration with a film institute and professional actors, we filmed each scene (except neutral) at three different emotional intensities. Then, we administered these intensity version scenes to 240 cognitively and mentally healthy participants, equally distributed in sex and age, ranging from 35 to 92 years.

Results: By applying Rasch analysis, the one intensity version per scene was selected that showed neither floor nor ceiling effects in participants whose ability in ER was in the medium range.

Conclusions: We have developed the first German-language TASIT-EET adaption, i.e., the "Basel Version of the Awareness of Social Inference Test - Emotion Recognition" (BASIT-ER). The BASIT-ER comprises the strengths of the TASIT-EET, while it overcomes its limitations such as inconsistencies in cinematic realization, low sensitivity in ER deficit detection, and the quite simple response format. Next, the BASIT-ER needs to be validated in clinical populations with neuropsychological disorders. 
O04 Motor Symptom Asymmetry and Cognitive Decline Following Stn Dbs in Parkinson's Disease: A Long-Term Follow-Up Study

\author{
P. Voruz ${ }^{1}$, C. Haegelen ${ }^{2}$, F. Assal ${ }^{3}$, S. Drapier ${ }^{4}$, P. Sauleau ${ }^{5}$, M. Vérin ${ }^{6}$, J. Péron ${ }^{1}$ \\ 1 Clinical and Experimental Neuropsychology Laboratory, Faculty of Psychology, Geneva, \\ Switzerland \\ 2 Neurosurgery Department, Pontchaillou University Hospital, Rennes, France \\ 3 Neurology Department, Geneva University Hospitals and Faculty of Medicine, Geneva, \\ Switzerland \\ 4 Behavior and Basal Ganglia' research unit, University of Rennes 1, France \\ 5 Behavior and Basal Ganglia' research unit, University of Rennes 1, France \\ 6 Neurology Department, Pontchaillou University Hospital, Rennes, France
}

Introduction: The aim of the present study was to investigate whether changes in global cognitive performances of patients with Parkinson's disease (PD) after STN-DBS were related to motor symptom asymmetry. As reported by our group (1), motor symptom asymmetry at baseline could predict emotional outcome in STN-DBS patients but its role on cognitive impairment has not been demonstrated yet.

Methods: 26 patients with PD [13 left-sided motor symptoms patients (LPD) and 13 rightsided motor symptoms patients (RPD)], who had undergone surgery for bilateral STNDBS between 2004 and 2015. They were followed up over a 5-year period, with four assessments at 6,12, 36 and 60 months post-DBS. These patient subgroups were compared with 13 healthy controls (HC) matched for mean age, gender, handedness and socioeducational level. Given our data a non-parametric statistical analysis was performed. For intergroup comparisons, we ran Kruskal Wallis tests, and if these were significant, we conducted Mann Whitney U tests, with Bonferroni correction. To determine the prevalence of scores indicating presence of MDRS, we compared each score individually against robust norms for the different ages carried out on the general population and considered any score below the 5 th percentile to be below the norm.

We observed a long-term deterioration in global cognitive performances RPD, whereas LPD performance remained statistically stable. Moreover, this cognitive deterioration in RPD was mainly driven by worsening memory and initiation subscores performance. The onset of cognitive decline in RPD was correlated with the worsening of motor symptoms, which were more bilateral in RPD even prior to DBS.

Result: Our results support a previous neuroimaging data of earlier left cortical atrophy in PD (2). The deterioration in nigrostriatal structures appeared to be greater in the left hemisphere (i.e., in RPD), leading to a worsening of performance and even the development of cognitive impairment, as suggested by the higher prevalence of among RPD at 60 months post-DBS $(38.46 \%)$, compared to the LPD $(23.08 \%)$. The current results, highlighting a left hemisphere vulnerability to neurodegeneration, open up a new avenue for research in the field of personalized medicine and support the notion that motor symptom asymmetry, together with right-sided motor symptoms at disease onset, could be a risk factor for the development of cognitive impairment associated with PD following STN-DBS.

O05 Visit-to-Visit Blood Pressure Variability, White Matter Integrity and Cognitive Decline in Cerebral Amyloid Angiopathy

L. Sveikata ${ }^{1}$, M.C. Zanon Zotin ${ }^{2}$, D. Schoemaker ${ }^{2}$, V. Perosa ${ }^{2}$, M. Etherton ${ }^{2}$, Y. Ma ${ }^{3}$, A. Charidimou ${ }^{2}$, S.M. Greenberg ${ }^{2}$, A. Viswanathan ${ }^{2}$

1 Massachusetts General Hospital, Harvard Medical School; Division of Neurology, Department of Clinical Neurosciences, Geneva University Hospitals

2 Massachusetts General Hospital, Harvard Medical School, Boston, USA 
Background: Emerging evidence suggests that blood pressure variability (BPV) may contribute to cerebral small vessel disease progression and cognitive impairment beyond the deleterious effects of elevated blood pressure, but the mechanisms remain largely unknown. $(1,2)$ This study investigates if BPV is associated with WM microstructural integrity and the slope of cognitive decline in elderly individuals with cerebral amyloid angiopathy (CAA), a well-characterized type of SVD.

Methods: We recruited 131 non-demented individuals $(73.3 \pm 7.0 \mathrm{y}, 33 / 101$ female/male, MMSE $27.5 \pm 2.0$ ) from the MGH memory-clinic with and without possible/probable CAA (24 and 50 patients respectively) according to modified Boston criteria. All participants underwent detailed neuropsychological testing, including global cognition, memory, language, processing speed and attention, and executive function, as well as a $3.0 \mathrm{~T}$ research MRI. Visit-to-visit BPV was assessed using the coefficient of variation derived from serial outpatient BP measures during a 5-year interval. A novel diffusion tensor imaging marker-peak width of skeletonized mean diffusivity (PSMD)-was used to evaluate the WM integrity. (3) Using linear regression models, we assessed the association of PSMD with BPV, adjusted for the number of blood pressure measures, age, sex, hypertension, usage of hypertensive medications, diabetes, smoking, and body mass index.

Results: We found a significant association between loss of WM integrity and high systolic BPV $(b=0.43, p<0.001)$, adjusted for mean BP. The association remained significant in the regression model adjusted for age, and standard cardiovascular risk factors $(b=0.41$, $p<0.001)$. Furthermore, the association of BPV with WM integrity was stronger when CAA was present $(B=7.58, p$ for interaction $<0.001)$. Higher $B P V$ was associated with worse executive function in CAA patients at baseline $(b=0.33, p=0.010)$ and at 2-year follow-up ( $b=-0.47, p=0.004)$, adjusted for age and baseline function.

Conclusions: Our findings show that visit-to-visit BP fluctuations are associated with the loss of WM microstructural integrity and cognitive decline. The association between BPV and WM integrity might in part be driven by CAA pathology. Better control of BPV could be a novel therapeutic target to slow cognitive decline over time. Further studies are warranted to disentangle the relationship between BP fluctuations, microvascular injury, and cognitive impairment.

\section{O06 Regular Exercise and Brain Health in Patients with Atrial Fibrillation}

E. Herber ${ }^{1}$, S. Aeschbacher ${ }^{1}$, F. Schwendinger ${ }^{2}$, M. Coslovsky ${ }^{2}$, E. Hennings ${ }^{1}$, A. Gasser ${ }^{1}$, M. Di Valentino ${ }^{3}$, T. Reichlin ${ }^{4}$, N. Rodondi ${ }^{5}$, S. Netzer ${ }^{5}$, J.H. Beer ${ }^{6}$, A. Stauber ${ }^{7}$, A. Müller ${ }^{7}$, T. Sinnecker ${ }^{1}$, E. Rigamonti ${ }^{8}$, P. Ammann ${ }^{9}$, D. Conen ${ }^{10}$, M. Kühne ${ }^{1}$, S. Osswald ${ }^{1}$, L. Bonati ${ }^{1}$

1 Universitätsspital Basel, Switzerland

2 Universität Basel, Switzerland

Ente Ospedaliero Cantonale, Bellinzona, Switzerland

4 Inselspital—Universitätsspital Bern, Switzerland

5 Inselspital-Universitätsspital Bern, Berner Institut für Hausarztmedizin (BIHAM), Switzerland

Kantonsspital Baden AG, Switzerland

7 Stadtspital Triemli Zürich, Switzerland

Ente Ospedaliero Cantonale, Ospedale Regionale di Lugano, Switzerland

9 Kantonsspital St. Gallen, Switzerland

10 Population Health Research Institute, McMaster University, Hamilton, Canada

Background: Many patients with atrial fibrillation (AF) have vascular brain lesions, which may impact cognitive function. Whether regular exercise is associated with brain lesions 
and cognitive function in patients with AF is unknown and was, therefore, the aim of this analysis.

Methods: Patients from the multicenter Swiss atrial fibrillation cohort study (Swiss-AF) with brain magnetic resonance imaging at baseline, neurocognitive testing, and data on physical activity were included in this cross-sectional analysis. We defined regular exercise as activities such as jogging, cycling, aerobic, or ball sports (at least once a week). Vascular brain lesions (ischemic infarcts, white matter lesions, and microbleeds) and total brain volume were evaluated by trained radiologists. The cognitive construct score was calculated from the entire neurocognitive test battery to describe global cognitive function. Results: We included 1490 patients with a mean age of $72 \pm 9$ years. 392 (26\%) were females and 730 patients (49\%) engaged in regular exercise activities. Using multivariable-adjusted logistic regression analysis, regular exercise was associated with a lower prevalence of ischemic brain infarcts (odds ratio (OR) 0.77, 95\%CI 0.61-0.96, $p=0.02$ ) and of moderate to severe white matter lesions (OR $0.77,95 \%$ CI $0.61-0.97, p=0.02$ ). Furthermore, regular exercise was associated with a larger total brain volume $(\beta$-coefficient $0.01,95 \% \mathrm{CI} 0.00$ $0.01, p=0.03)$ and by trend, with decreased white matter lesion volume $(\beta-0.12,95 \% \mathrm{CI}$ $-0.25-0.01, p=0.06)$. The cognitive construct score was significantly higher in patients doing regular exercise activities when adjusted for demographic and clinical characteristics, education and the presence of brain infarcts $(\beta 0.07,95 \%$ CI $0.03-0.12, p=0.001)$.

Conclusions: In patients with $\mathrm{AF}$, regular exercise was associated with larger brain volume, less ischemic brain infarcts and white matter disease, as well as better neurocognitive performance. Therefore, patients with AF should be encouraged to remain physically active and engage in regular exercise activities.

\section{O07 Multiparametric Model for Prediction of Active and Occult Malignancy in} Stroke Patients

M. Beyeler ${ }^{1}$, B. Birner ${ }^{1}$, M. Branca ${ }^{2}$, T. Meinel ${ }^{1}$, J. Vynckier ${ }^{1}$, E. Buffle ${ }^{3}$, A. Scutelnic ${ }^{1}$, M. Heldner ${ }^{1}$, P. Bücke ${ }^{1}$, D. Seiffge ${ }^{1}$, P. Mordasini ${ }^{4}$, T. Dobrocky ${ }^{4}$, E. Piechowiak ${ }^{4}$, J. Kaesmacher ${ }^{4}$, J. Gralla ${ }^{4}$, M. Arnold ${ }^{1}$, U. Fischer ${ }^{1}$, T. Pabst ${ }^{5}$, M. Berger ${ }^{5}$, S. Jung ${ }^{1}$

1 Department of Neurology, Inselspital, Bern University Hospital, and University of Bern, Switzerland

2 CTU Bern, Institute of Social and Preventive Medicine, University of Bern, Bern, Switzerland

3 Department of Cardiology, Inselspital, Bern University Hospital, and University of Bern, Switzerland

4 Department of Diagnostic and Interventional Neuroradiology, Inselspital, Bern University Hospital, and University of Bern, Switzerland

5 Department of Oncology, Inselspital, Bern University Hospital, and University of Bern, Switzerland

Background and Objective: To detect occult malignancy which determines early treatment decisions, patient outcome, and guidance of secondary prevention as a stroke etiology, we aimed to develop a multiparametric predictive clinical score for occult malignancy based on associated biomarkers.

Material \& Methods: Single-center cross-sectional study including consecutive first-ever ischemic stroke patients treated between July 2017 and November 2018. After identification of patients with a diagnosis of active malignancy before index-stroke (known malignancy) and 1-year thereafter (occult malignancy), Lasso analysis of logistic regression and crossvalidation by bootstrapping were used to determine discrimination and calibration of prespecified clinical variables and biomarkers for active malignancy. A comparison between known and occult malignancies enabled to exclude variables not associated with occult malignancy. Based on the remaining variables, we developed the OCCULT-5 Score. 
Results: Among 1001 patients with first ischemic stroke, 61 patients (6.09\%) had an active malignancy. Out of those, 39 patients $(64 \%)$ had a known malignancy and 22 patients $(36 \%)$ had an occult malignancy. After exclusion of variables found only in patients with known malignancy (CRP and $\mathrm{Hb}$ ) or too many missing values ( $\mathrm{LDH}$ ) we developed a five-item score ( 1 point each). The final score includes age $\geq 77$ years old, embolic stroke of undetermined source, multi-territorial infarcts, D-dimer level $\geq 820 \mu / \mathrm{gL}$, and female sex. The area under the Receiver Operating Characteristic curve is 0.7 (95\%CI 0.57-0.82). A score of $\geq 3$ is associated with a sensitivity of $64 \%$, a specificity of $73 \%$, a positive likelihood ratio of 2.35, and a negative likelihood ratio of 0.49 with occult malignancy in our study population.

Conclusions: The combination of malignancy-related stroke biomarkers increases the prediction of occult malignancy in stroke patients. If validated in other cohorts, the OCCULT-5 Score may improve the early detection of occult malignancy in stroke patients and may thus have major impact on survival in this population.

\title{
O08 Migraine Symptoms Are Frequent in Acute Ischemic Stroke
}

\author{
A. Scutelnic ${ }^{1}$, L.A. Kreis ${ }^{1}$, M. Beyeler ${ }^{1}$, H. Arsany ${ }^{2}$, J. Kaesmacher ${ }^{2}$, T.R. Meinel ${ }^{1}$, \\ U. Fischer ${ }^{3}$, M. Arnold ${ }^{1}$, M.R. Heldner ${ }^{1}$, H.P. Mattle ${ }^{1}$, C. Schankin ${ }^{4}$, S. Jung ${ }^{1}$ \\ 1 Inselspital-Universitätsspital Bern - Universitätsklinik für Neurologie, Switzerland \\ 2 Inselspital-Universitätsspital Bern - Universitätsinstitut für Diagnostische und Inter- \\ ventionelle Neuroradiologie, Switzerland \\ 3 Department of Neurology and Stroke Center, University Hospital and University of \\ Basel, Switzerland \\ 4 Inselspital-Universitätsspital Bern, Switzerland
}

Background: Irritative and spreading symptoms with gradual onset are characteristic of migraine aura. Current diagnostic criteria for transient ischemic attack (1) exclude presence of such symptoms. However, cortical spreading depression, the pathophysiologic basis of migraine aura, has been found in ischemic stroke with high incidence (2), suggesting an overlap of clinical manifestation of ischemic brain events and migraine aura. Only one study from 1968 with 120 AIS patients addressed the detailed phenomenology of stroke symptoms yet.

Aim: To systematically compare symptoms in AIS with MWA with regard to onset, duration, spread and quality.

Methods: 353 consecutive patients with AIS confirmed by imaging and 350 patients with MwA, classified according to ICHD-3 criteria were included. Type (irritative/deficit), onset-time (sudden/gradual), duration of symptoms and headache were assessed by structured questionnaire.

Results: In the stroke-group, eighty-three (23.75\%) patients had visual disturbance, of whom $32(39 \%)$ had irritative symptoms only and $11(13 \%)$ both irritative and deficit symptoms, the remainder [40 (48\%)] having deficit symptoms only. One hundred fifty three $(43 \%)$ patients had sensory disturbance, $48(31 \%)$ of whom reported irritative symptoms only and $31(20 \%)$ both irritative and deficit symptoms, the remainder [74 (49\%)] reporting deficit symptoms only. Eighteen (22\%) patients with visual disturbance reported a gradual onset of symptoms $>5 \mathrm{~min}$. Thirty five $(23 \%)$ patients with sensory disturbance reported a gradual onset of symptoms > $5 \mathrm{~min}$. Even 44/199 (22\%) patients with paresis reported a gradual onset of $>5 \mathrm{~min}$. Of 204 (58\%) patients who had more than one symptom, $105(52 \%)$ had symptoms occurring in succession. Lastly, the C-criterium of the ICHD-3 classification for MwA was formally fulfilled in 135 (38\%) in our stroke-cohort.

Conclusions: Migraine symptoms in AIS and stroke symptoms in MWA are much more common than traditionally thought. This has major impact on the diagnosis of stroke and TIA and its differentiation from migraine aura. 
O09 Central Pain after Thalamic Stroke Is Associated with Hypaesthesia for Light Touch and Mechanical Hyperalgesia

\author{
L. Enz ${ }^{1}$, K. Berati ${ }^{1}$, S. Magon ${ }^{2}$, T. Sprenger ${ }^{3}$, A. Papadopoulou ${ }^{4}$ \\ 1 University Hospital Basel, Switzerland \\ 2 Roche Basel, Switzerland \\ 3 DKD HELIOS Klinik Wiesbaden, Switzerland \\ 4 University Hospital Basel/University of Basel, Switzerland
}

Background and aim: Chronic post stroke pain (CPSP) is a disabling disorder that can follow ischemic or hemorrhagic stroke if the somatosensory system is affected. Currently, treatment is typically initiated once the pain has occurred and the disorder is often difficult to treat. We are aiming at detecting sensory phenotypes associated with CPSP after thalamic stroke. Such phenotypes may allow an early identification of patients at risk for CPSP.

Material and methods: Standardized quantitative sensory testing (QST) of the affected side was conducted in 16 patients with CPSP after thalamic stroke, 14 patients with thalamic stroke without pain ("no pain" group) and 12 healthy controls (HC). The assessment was performed on average $54 \pm 63$ months (CPSP) or $62 \pm 19$ months (no pain group) after stroke. Assessed qualities included: mechanical, warm and cold detection thresholds, thermal sensory limen (TSL), mechanical, cold and heat pain thresholds, dynamic mechanical allodynia, wind-up ratio, vibration threshold, and pressure pain threshold. Statistical analysis was performed with one-way analysis of variance followed by tukey post-hoc correction.

Results: Mechanical detection thresholds (to von Frey hairs) were significantly higher in the CPSP group compared to the no pain group (adj. $p=0.032$, Cohens $\mathrm{d}=0.902$ ) and to the HC (adj. $p=0.037$, Cohens $\mathrm{d}=1.18$ ), indicating hypesthesia to light touch in the CPSP group. Mechanical pain thresholds (to PinPrick stimulators) were lower in the CPSP group compared to the no pain group (adj. $p=0.015$, Cohens $\mathrm{d}=-1.08$ ), but did not differ from $\mathrm{HC}$ (adj. $p=0.76$, Cohens $\mathrm{d}=0.26$ ). All differences remained significant after correcting for sex and age. A combination of mechanical detection threshold and mechanical pain threshold distinguished the CPSP and the no pain group with a specificity of $76.9 \%$, a sensitivity of $81.3 \%$ and an accuracy of $79.3 \%$.

Conclusions: Patients with CPSP after thalamic stroke are characterized by hypesthesia to light touch and are more sensitive to mechanical pain than patients with thalamic stroke without pain. Interestingly, allodynia, temperature-, vibration- and pressure-perception were similar between the two groups. Systematic screening of patients with thalamic stroke using QST may help identifying patients at risk. Such patients may in the future be candidates for prophylactic/early treatment and/or study interventions.

O10 Encephalopathies Associated with Severe COVID-19 Present Neurovascular Unit Alterations without Evidence for Strong Neuroinflammation

\title{
R. Bernard-Valnet, S. Perriot, M. Canales, B. Pizzarotti, L. Caranzano, M. Castro-Jimenez, J. Epiney, S. Vijiala, P. Salvioni-Chiabotti, A. Anichini, A. Salerno, M. Perreau, G. Pantaleo, G. Greub, R. Du Pasquier
}

\section{CHUV Centre Hospitalier Universitaire Vaudois (CHUV), Lausanne, Switzerland}

Objective: Coronavirus disease (COVID-19) has been associated with a large variety of neurological disorders. However the mechanisms underlying these neurological complications remain elusive. In this study we aimed at determining whether neurological symptoms were caused by SARS-CoV-2 direct infection or by either systemic or local pro-inflammatory mediators. 
Material and methods: In this cross-sectional study, we checked for SARS-CoV-2 RNA by RT-qPCR, SARS-CoV-2-specific antibodies and for 49 cytokines/chemokines/growth factors (by Luminex) in the cerebrospinal fluids (CSF) \pm sera of a cohort of 22 COVID-19 patients with neurological presentation and 55 neurological control patients (inflammatory [IND], non-inflammatory [NIND], multiple sclerosis [MS]).

Results: We detected anti-SARS-CoV-2 IgG in patient with severe COVID-19 with signs of intrathecal synthesis for some of them. Of the four categories of tested patients, the CSF of IND exhibited the highest level of cytokines, chemokines and growth factors. In contrast, COVID-19 patients did not present overall upregulation of inflammatory mediators in the CSF. However, the CSF of patients with severe COVID-19 (ICU patients) exhibited higher concentrations of CCL2, CXCL8, and VEGF-A in the CSF than patients with a milder form of COVID-19. In addition, we could show that intrathecal CXCL8 synthesis was linked to an elevated albumin ratio and correlated to the increase of peripheral inflammation (serum HGF and CXCL10).

Conclusions: Our results do not indicate active replication of SARS-CoV-2 in the CSF or signs of massive inflammation in the CSF compartment, but highlight a specific impairment of the neurovascular unit linked to intrathecal production of CXCL8.

O11 Paramagnetic Rim Lesions in MS Are Characterized by Heterogeneous Damage and Inflammatory Activity: A Combined T1 Relaxometry-Diffusion Study

\section{Barakovic ${ }^{1}$, R. Galbusera ${ }^{1}$, R. Rahmanzadeh ${ }^{1}$, M. Weigel ${ }^{1}$, P. Lu ${ }^{1}$, E. Bahn ${ }^{2}$, P. Sati ${ }^{3}$, P. Maggi ${ }^{4}$, S. Schiavi ${ }^{5}$, A. Daducci ${ }^{5}$, L. Kappos ${ }^{6}$, J. Kuhle ${ }^{7}$, L. Gaetano ${ }^{8}$, C. Stadelmann ${ }^{2}$, S. Magon ${ }^{9}$, C. Granziera ${ }^{1}$}

1 Translational Imaging in Neurology Basel, Department of Medicine and Biomedical Engineering, University Hospital Basel and University of Basel, Switzerland

2 Institute of Neuropathology, University Medical Center, Zurich, Switzerland

3 Translational Neuroradiology Section, National Institute of Neurological Disorders and Stroke, Maryland, USA

4 Department of Neurology, Lausanne University Hospital, Switzerland

5 Department of Computer Science, University of Verona, Italy

6 Research Center for Clinical Neuroimmunology and Neuroscience (RC2NB) Basel, University Hospital Basel and University of Basel, Switzerland

7 Neurologic Clinic and Policlinic, Switzerland, Departments of Medicine, Clinical Research and Biomedical Engineering, University Hospital Basel and University of Basel, Switzerland

8 F. Hoffmann-La Roche Ltd, Basel, Switzerland

9 Pharmaceutical Research and Early Development, Roche Innovation Center Basel, F. Hoffmann-La Roche Ltd., Switzerland

Introduction: Lesions presenting a paramagnetic rim on susceptibility-sensitive MRI (paramagnetic rim lesions-PRL) are characterized by chronic inflammatory activity (DalBianco et al., 2017) and are associated with increased disability (Absinta et al. 2019) and neuroaxonal damage (Maggi et al. 2019) in patients with Multiple Sclerosis (MS). Histopathologically, PRL feature a rim of activated microglia/macrophages at the edge, and various degrees of inflammatory demyelinating activity and infiltration of activated microglia/macrophages in the lesion core.

In this work, we aimed at disentangling some characteristics of PRL by combining a measure of extra-cellular restricted fractions derived from multi-shell diffusion data with the quantification of mean T1 relaxation times from MP2RAGE. The diffusion model was chosen based on histopathological characteristics of PRL lesions, which feature a pronounced axonal damage (and hence a strong loss of anisotropy) in the lesion core. 
Methods: We acquired MRI images of 102 MS patients on a 3T MRI system. Among those, 46 patients presented PRL. Diffusion-weighted MRI (DW-MRI) data were acquired with resolution $1.8 \mathrm{~mm}$ isotropic and the following b-values $[0,700,1000,2000,3000] \mathrm{s} / \mathrm{mm}^{2}$. MP2RAGE images (Marques et al. 2010) with $1 \mathrm{~mm}$ isotropic resolution, provided T1 relaxometry maps. 3D FLAIR with isotropic resolution $(1 \mathrm{~mm})$ was used for lesion segmentation. Results: We performed a voxel-wise, and lesion-wise analysis of the DWI-based stick-ballsphere fractions and qT1 in 226 PRL identified in vivo in MS patients. The voxel-wise analysis, which included all PRL voxels, showed a Pearson correlation coefficient of -0.63 between sphere fraction and qT1.

Conclusions: Using measures of both qT1 and sphere-fraction derived from diffusion MRI, we provide evidence that PRL structurally heterogeneous in vivo in MS patients. Short qT1 in PRL corresponded to higher values of sphere fractions, which might correspond to a high concentration of activated microglia/macrophages of highly inflammatory PRL or eventually to axonal spheroids due to ongoing axonal damage. On the other hand, PRL lesions with long qT1 values are associated to lower sphere-fractions and higher fractions of ball-fractions in PRL, suggesting an extensive tissue damage but also the scarce presence of inflammatory cells/axonal spheroids.

\section{O12 Dimethyl Fumarate Alters Gut Microbiota Metabolism in Multiple Sclerosis}

\section{Diebold, S. Purushothaman, T. Roloff, E. Pössnecker, M. Meola, R. Lindberg, J. Kuhle, T. Derfuss, A. Egli, A. Pröbstel}

\section{University Hospital Basel, Switzerland}

Background and Objective: Multiple sclerosis (MS) ranges among the most prominent diseases, in which a crucial pathophysiological role of gut microbiota is discussed. It remains unknown, how disease-modifying treatments alter gut microbiota composition and whether microbiological taxa shape treatment response and side-effects. Notably, dimethyl fumarate (DMF), an approved oral treatment of MS, frequently causes self-limited gastrointestinal side-effects, and is reported to alter the metabolic activity in immune cells. In this study, we aim to explore the associations between the metabolic properties of DMF and the gut microbiome and whether distinct microbial signatures can be linked to clinical response and side-effects.

Methods: We prospectively collected serum and stool samples before first intake and after 3 months of DMF treatment from MS patients. All samples were timely processed and cryopreserved until analysis. Stool samples were assessed for their gut microbiome composition using the QIAseq 16S/ITS screening panel (QIAGEN), sequenced on a MiSeq (Illumina). Operational taxonimic units abundances were calculated and pathways enrichments were inferred. Serum samples were characterized for metabolomics by mass spectrometry. Clinical assessment of all patients included neurological status, relapse rate, magnetic resonance imaging, side-effects and immune cell phenotyping using multi-color flow cytometry allowing biological data interpretation in correlation to clinical endpoints. Results: A cohort of 20 patients completed follow-up in this study. Longitudinal assessment of gut microbiota composition showed distinct alterations across patients under DMF treatment. Notably, predicted metagenome functional states of the detected microbiota further indicated relevant treatment associated alterations in several metabolic pathways including glycolysis and TCA cycle. Serum metabolomics showed the most significant longitudinal changes under treatment for late TCA cycle metabolites. Importantly, intra-individual differences in microbial composition correlated with treatment side-effects. Further integration of microbiome and metabolome data as well as clinical and immunological outcomes is ongoing.

Conclusions: We show that metabolic properties of DMF reach beyond immune cells and relevantly alter the gut microbiome. Further analysis will distinguish how this impact on gut microbiota determines side effects and shapes therapeutic response to DMF. 
O13 Continuous High Frequency Deep Brain Stimulation in the Anterior Nucleus of the Thalamus Increases Slow Wave Sleep

\author{
J. Bünzli ${ }^{1}$, L. Stieglitz ${ }^{2}$, C. Baumann ${ }^{2}$, E. Werth ${ }^{2}$, P. Hilfiker ${ }^{3}$, L. Imbach ${ }^{3}$ \\ 1 Department of Health Sciences and Technology, ETH Zurich, Switzerland \\ 2 University Hospital Zürich, Switzerland \\ 3 Schweizerisches Epilepsiezentrum, Zurich, Switzerland
}

Previous studies showed that intermittent deep brain stimulation (DBS) on the anterior nucleus of the thalamus (ANT) might result in disrupted sleep architecture. Here, we investigated the impact ANT-DBS on sleep in epilepsy patients under continuous DSB in a multicenter cross-over study in ten patients. Sleep stage distribution, delta power, delta energy, total sleep time (TST), were assessed in standardized polysomnographic (PSG) investigations before and after DBS. In contrast to previous studies, we found no disruption of sleep architecture or sleep stage distribution $(p=0.76)$. On the contrary, we observed more consolidated and deeper slow wave sleep (SWS) under continuous high-frequency DBS. In particular, delta power and delta energy exhibited a significant increase postDBS as compared to baseline $\left(36.67 \pm 13.68 \mu \mathrm{V} 2\right.$ and $799.86 \pm 407.56 \mu \mathrm{V}^{*}{ }^{*}$, respectively, $p<0.001)$. Furthermore, the observed increase in delta power was dependent from the stimulation point within the ANT: we observed higher delta power $(76.00 \pm 9.14 \mu \mathrm{V} 2$ lower vs. $114.58 \pm 43.12 \mu \mathrm{V} 2$ upper, $p<0.001)$ and higher delta energy $\left(2023.70 \pm 562.40 \mu \mathrm{V} 2{ }^{*} \mathrm{~s}\right.$ lower vs. $2512.31 \pm 1085.24 \mu \mathrm{V} 2{ }^{*}$ s upper, $\left.p<0.001\right)$ in more cranial stimulation as compared to caudal or mid-range contacts. Under continuous DBS, significantly less nocturnal discharges occurred than without $(838.9 \pm 1952.2 \mathrm{~s}$ pre vs. $561.1 \pm 1446.7 \mathrm{~s}$ post, $p<0.001)$. Intriguingly, high stimulation amplitudes (above $3 \mathrm{~V}$ ) showed both lower delta power and a lower delta energy $(p<0.001)$, indicating a saturation effect. In conclusion, our findings suggest that continuous ANT-DBS in the cranial part leads to more consolidated SWS. From a clinical perspective, patients with sleep disruption under cyclic ANT-DBS could benefit from cranial and continuous stimulation of the ANT.

\title{
O14 Asymmetric Sleep in Patients with Epilepsy
}

\section{Sheybani, M. Seeck, S. Vulliémoz \\ University Hospital of Geneva, Switzerland}

Context and Objectives: When healthy subjects sleep in a novel environment, delta power is decreased in the left hemisphere. This effect dissipates during the second night in the same environment (1). This decrease is interpreted as a protective mechanism that provides a "vigil hemisphere" in an unfamiliar environment. In parallel, it is known that patients with focal epilepsy present an increased delta activity in the hemisphere where the epileptic focus is localized (2). Here, we wanted to address the conflicting situation in which patients with left focal epilepsy would thus be expected to show both an increase in delta power in the left hemisphere (i.e., in the epileptogenic hemisphere) and a decrease in delta power in the left hemisphere (i.e., in the "vigil hemisphere") during sleep in a novel environment. Materials and Methods: We measured delta power $(0.5-4 \mathrm{~Hz})$ during the first two consecutive nights of 74 patients who underwent long-term ( $\geq 2$ nights) EEG recording for presurgical investigation of focal, pharmacoresistant epilepsy. We compared the asymmetry index (left - right/left + right) of delta power during the first and second nights in patients with left- vs. right-hemispheric epilepsy. We then used the same index for spindle density (i.e., spindle per minute) across the two nights. These indices were then used to test whether they could cluster patients with left- vs. right-hemispheric epilepsy.

Results: In patient with right focal epilepsy, delta power is decreased over the left hemisphere during the first but not the second night in a novel environment, similarly to healthy 
controls. In patients with left focal epilepsy, this asymmetry is lacking. Spindle density shows a clear asymmetry in favour of the non-epileptogenic hemisphere, but no difference between the first and second night. Finally, these parameters can indeed cluster above chance level patients with left- vs. right focal epilepsy.

Conclusions: Our results establish that patients with left focal epilepsy fail to re-organize a transient "night watch hemisphere" (1) contralaterally. The asymmetry of spindle density in favour of the non-epileptogenic hemisphere is highly interesting, in the light of evidence showing that spindle and epileptic activities compete for the same brain circuits (3). Finally, we propose a new and innovative index to cluster patients with left- vs. right focal epilepsy, which could prove to be a useful tool in presurgical investigation of epilepsy.

\section{O15 Electroencephalography of Mechanically Ventilated Patients at High Risk of Delirium}

\section{E. Eskioglou ${ }^{1}$, C. Iaquaniello ${ }^{2}$, V. Alvarez ${ }^{3}$, S. Rüegg ${ }^{4}$, K. Schindler ${ }^{5}$, A.O. Rossetti ${ }^{1}$, M. Oddo ${ }^{1}$}

1 CHUV Centre Hospitalier Universitaire Vaudois (CHUV), Lausanne, Switzerland

2 University of Milan;

3 Hôpital de Sion, Switzerland

4 Basel University Hospital, Switzerland

5 Inselspital-Universitätsspital Bern, Switzerland

Context and Objectives: Neurophysiological exploration of ICU delirium is limited. Here, we examined EEG characteristics of medical-surgical critically ill patients with new onset altered consciousness state at high risk for ICU delirium.

Materials and Methods: Pre-planned analysis of non-neurological mechanically ventilated medical-surgical ICU subjects, who underwent a prospective multicenter randomized, controlled EEG study (NCT03129438, April 2017-November 2018). EEG characteristics, according to the 2012 ACNS nomenclature, included background activity, rhythmic periodic patterns/epileptic activity, amplitude, frequency, stimulus-induced discharges, triphasic waves, reactivity and NREM sleep. We explored EEG findings in delirious vs. non-delirious patients, specifically focusing on presence of burst-suppression and rhythmic periodic patterns (ictal-interictal continuum), and ictal activity.

Results: We analyzed 91 patients (median age, 66 years) who underwent EEG because of new onset altered consciousness state at a median 5 days from admission; 42 patients developed delirium (46\%). Burst-suppression (10 vs. $0 \%, p=0.02)$, rhythmic/periodic patterns $(43 \%$ vs. $22 \%, p=0.03)$ and epileptiform activity ( 7 vs. $0 \%, p=0.05)$ were more frequent in delirious vs. non-delirious patients. The presence of at least one of these abnormal EEG findings (32/91 patients; $35 \%$ ) was associated with a significant increase in the likelihood of delirium ( 42 vs. $15 \%, p=0.006$ ). Cumulative dose of sedatives and analgesics, as well as all other EEG characteristics, did not differ significantly between the two groups.

Conclusions: In mechanically ventilated non-neurological critically ill patients with new onset alteration of consciousness, EEG showing burst-suppression, rhythmic or periodic patterns, or seizures/status epilepticus indicate an increased risk of ICU delirium.

P01 Serum S-100B Adds Incremental Value for the Prediction of Symptomatic Intracranial Hemorrhage and Symptomatic Brain Edema after Acute Ischemic Stroke: Data from the Multicentre BIOSIGNAL-Cohort Study

T. Honegger ${ }^{1}$, J. Schweizer ${ }^{1}$, A. Bicvic ${ }^{1}$, L.P. Westphal ${ }^{1}$, V.C. Schütz ${ }^{1}$, C. Inauen ${ }^{1}$, T. Pokorny ${ }^{1}$, K. Bracher ${ }^{1}$, M. Arnold ${ }^{2}$, U. Fischer ${ }^{2}$, L. Bonati ${ }^{3}$, G.M. De Marchis ${ }^{3}$, K. Nedeltchev ${ }^{4}$, T. Kahles ${ }^{4}$, C. Cereda ${ }^{5}$, G. Kägi ${ }^{6}$, J. Montaner ${ }^{7}$, A. Bustamante ${ }^{8}$, 
E. Palà ${ }^{9}$, G. Ntaios ${ }^{10}$, C. Foerch ${ }^{11}$, A. Luft ${ }^{1}$, K. Spanaus ${ }^{1}$, A. von Eckardstein ${ }^{1}$, M Arnold ${ }^{1}$, M Katan Kahles ${ }^{1}$

1 Universitäts Spital \& Universität Zürich, Switzerland

2 Inselspital-Universitätsspital Bern, Switzerland

3 Universitätsspital Basel, Switzerland

4 Kantonsspital Aarau, Switzerland

5 Ente Ospedaliero Cantonale, Ospedale Regionale di Lugano, Switzerland

6 Kantonsspital St. Gallen, Switzerland

7 Hospital Universitari Vall d'Hebron, Barcelona, Spain

8 Hospital Universitari Germans Trias i Pujol, Barcelona, Spain

9 Vall d'Hebron Institute of Research, Universitat Autònoma de Barcelona, Spain

10 University of Thessaly Larissa, Greek

11 University Hospital Frankfurt am Main, Germany

Background and Purpose: Early identification of patients developing symptomatic intracranial hemorrhage $(\mathrm{sICH})$ and symptomatic brain edema (sBE) after acute ischemic stroke (AIS) is essential for clinical decision-making. Astroglial protein S-100B is a marker of blood-brain barrier disruption, which plays a role in the formation of sICH and sBE. In this study, we assessed the prognostic value of serum S-100B for the development of sICH and sBE.

Methods: Serum S-100B levels were measured on admission (within $24 \mathrm{~h}$ from symptom onset) in 1749 consecutive AIS patients from the multicenter BIOSIGNAL cohort study (mean age 72.0 years, $58.3 \%$ male). To determine sICH or sBE, follow-up neuroimaging was routinely performed in all patients receiving re-canalisation therapy or experiencing significant clinical worsening defined as an NIHSS increase of $\geq 4$ during hospital stay.

Results: Of the 1749 consecutively enrolled patients, 46 (2.6\%) developed sICH and 90 $(5.2 \%)$ developed sBE. After adjustment for established risk-factors, S-100B levels remained independently associated with both sICH (OR 3.41, 95\%CI 1.7-6.9, $p=0.001$ ) and sBE (OR $4.08,95 \%$ CI 2.3-7.1, $p<0.001$ ) in multivariable logistic regression models. Adding S-100B to the best clinical prediction model led to an improvement of AUC from 0.72 (95\%CI $0.65-0.78)$ to $0.75(95 \% \mathrm{CI} 0.68-0.82)(p=0.001)$ for sICH and from $0.78(95 \% \mathrm{CI} 0.73-0.83)$ to $0.81(95 \%$ CI 0.76-0.86) $(p<0.0001)$ for sBE (Figure 1$)$.

Conclusions: Serum S-100B levels are independently associated with the development of sICH and sBE, adding incremental prognostic value in AIS patients. Thus, S-100B may be clinically useful for early risk-stratification regarding acute stroke complications.

P02 Genetic Proxies for Pcsk9 Inhibition Associated with Lower Lipoprotein(A): Effects on Coronary Artery Disease and Ischemic Stroke

G.M. De Marchis ${ }^{1}$, T. Dittrich ${ }^{1}$, R. Malik ${ }^{2}$, A. Zietz ${ }^{1}$, L. Kriemler ${ }^{1}$, B. Ference ${ }^{3}$, M. Dichgans ${ }^{4}$, M. Georgakis ${ }^{4}$

1 Unispital Basel, Switzerland

2 Institute for Stroke and Dementia Research, University Hospital, Ludwig-MaximiliansUniversität LMU, Munich, Germany

3 Centre for Naturally Randomized Trials, University of Cambridge, Cambridge, United Kingdom

4 Institute for Stroke and Dementia Research, University Hospital, Ludwig-MaximiliansUniversität LMU, Munich, Germany

Background and Aims: Lipoprotein(a) concentration is associated with cardiovascular events independently of LDL-C. Post hoc analyses of clinical trials show that PCSK9 inhibitors might lower lipoprotein(a), but whether this effect contributes to reductions in 
cardiovascular risk remains unknown. In the absence of available lipoprotein(a)-reducing therapies, we aimed to assess whether genetically proxied PCSK9 inhibition influences lipoprotein(a) levels, and whether any such effect could mediate part of the effects of PCSK9 inhibitors on coronary heart disease (CAD) and ischemic stroke (IS).

Methods: We identified 12 genetic variants in the PCSK9 gene to be used as proxies (instruments) for PCSK9 inhibition based on association with LDL-C $\left(p<5 \times 10^{-8}, n=188,577\right)$ and performed mendelian randomization analyses.

Results: In our analyses, we found a $6 \%$ decrease in lipoprotein(a) levels (beta for loglipoprotein(a): $-0.061,95 \%$ CI: -0.085 to -0.037 ) per 1-SD decrement in LDL-C levels $(-39.8 \mathrm{mg} / \mathrm{dL})$. We then performed two-step mediation analyses based on data from the CardioGramPLUSC4D (60,801 CAD cases, 184,305 controls) and the MEGASTROKE Consortia (60,341 IS cases, 454,450 controls). We estimated a $0.8 \%$ reduction in the odds for CAD (OR: $0.992,95 \%$ CI: $0.988-0.996)$ and a $0.9 \%$ reduction in the odds for large artery atherosclerotic stroke (OR: 0.991, 95\%CI: 0.985-0.998) due to reductions in lipoprotein(a) levels through variation in the PCSK9 gene, corresponding to $1.8 \%$ and $3.3 \%$ of the total effects, respectively.

Conclusions: Genetically proxied PCSK9 inhibition is associated with lower lipoprotein(a) levels. However, this lipoprotein(a) reduction contributes only modestly to the total effects of genetic proxies for PCSK9 inhibitors on risk of CAD and IS.

P03 Reperfusion Therapies of Isolated Posterior Cerebral Artery Occlusion: An Analysis of the Swiss Stroke Registry (SSR)

F. Maulucci ${ }^{1}$, G. Disanto ${ }^{1}$, G. Bianco ${ }^{1}$, M. Pileggi ${ }^{2}$, G. Padlina ${ }^{3}$, U. Fischer ${ }^{3}$, D. Strambo ${ }^{4}$, P. Michel ${ }^{4}$, T. Kahles ${ }^{5}$, K. Nedeltchev ${ }^{5}$, U. Fisch ${ }^{6}$, L. Bonati ${ }^{6}$, G. Kägi ${ }^{7}$, A. Müller ${ }^{7}$, J.B. Escribano Paredes ${ }^{8}$, E. Carrera ${ }^{8}$, T. Nyffeler ${ }^{9}$, M. Bolognese ${ }^{9}$, S. Wegener ${ }^{10}$, A. Luft ${ }^{10}$, L. Schelosky ${ }^{11}$, F. Medlin ${ }^{12}$, B. Rodic ${ }^{13}$, A. von Reding ${ }^{13}$, N. Peters ${ }^{14}$, R. Backhaus ${ }^{14}$, S. Renaud ${ }^{15}$, M.L. Mono ${ }^{16}$, M. Psychogios ${ }^{17}$, J Kaesmacher ${ }^{18}$, P. Mordasini ${ }^{18}$, C. Cereda ${ }^{1}$

1 Stroke Center, Neurology, Neurocenter of Southern Switzerland

2 Stroke Center, Interventional and Diagnostic Neuroradiology, Neurocenter of Southern Switzerland

3 University Hospital Bern, Department of Neurology, Switzerland

4 Lausanne University Hospital, Stroke Center, Neurology Service, Switzerland

5 Kantonsspital Aarau, Department of Neurology, Switzerland

6 University Hospital Basel and University of Basel, Department of Neurology and Stroke Center, Switzerland

7 Kantonsspital St. Gallen, Department of Neurology, Switzerland

8 Hôpitaux Universitaires de Genève, Department of Neurology, Switzerland

9 Luzerner Kantonsspital, Center of Neurology and Neurorehabilitation, Switzerland

10 Universitätsspital Zürich, Neurology, Switzerland

11 Kantonsspital Münsterlingen, Division of Neurology, Switzerland

12 HFR Fribourg, Stroke Unit, Division of Neurology, Switzerland

13 Kantonsspital Winterthur, Neurology, Switzerland

14 Hirslanden Hospital, Department of Neurology and Stroke Center, Basel, Switzerland

15 Pourtalès Hospital, Division of Neurology, Neuchâtel, Switzerland

16 Stadtspital Waid und Triemli, Stroke Unit, Switzerland

17 University Hospital Basel, Department of Neuroradiology, Switzerland

18 University Hospital Bern, Inselspital, Neuroradiology, Switzerland

Background and Aims: There are few data about efficacy of endovascular treatment (EVT) and bridging therapy of acute ischemic stroke (AIS) due to isolated posterior cerebral artery 
occlusion (PCAO). We aimed to verify the functional outcome and safety of AIS patients with PCAO, treated with EVT or EVT + IVT (bridging), compared to IVT alone.

Results: From $n=17,968$ patients, 298 met the criteria. Propensity score method matched 81 in each group. Patients with IVT and EVT (of whom 69\% had bridging) showed comparable excellent outcome ( $44 \%$ vs. $33 \%$ respectively; aOR $=0.55,95 \% \mathrm{CI}=0.27-1.13$, $p=0.104)$. With both treatments, a substantial proportion of patients was independent at 90 days, and significantly higher in IVT alone (72\%) compared to EVT $(58 \%)(\mathrm{aOR}=0.30$, $95 \% \mathrm{CI}=0.12-0.72, p=0.007)$. Symptomatic ICH was non-significantly higher in IVT $(4.9 \%$ vs. $1.2 \% ; p=0.211)$. Mortality at 90 days was comparable between IVT and EVT ( $7.4 \%$ vs. $4.9 \% ; p=0.516)$. No significant differences were documented after stratification for more proximal site of occlusion compared to more distal (P1 vs. P2/P3).

Conclusions: In AIS due to PCAO, EVT and IVT appears similarly effective in terms of excellent outcome. IVT seems superior for reaching independence at 3 months. This aspect should be further investigated in future RCTs.

P04 A Case Report on Reversible Cerebral Vasoconstriction Syndrome and Transient Global Amnesia

\author{
F. Maibach ${ }^{1}$, A. Czaplinski ${ }^{1}$, N. Peters ${ }^{2}$, A. Paliantonis ${ }^{1}$ \\ 1 Hirslanden Zürich, Switzerland \\ 2 Hirslanden Zürich/Universität Basel, Switzerland
}

Reversible Cerebral Vasoconstriction Syndrome (RCVS) presents as severe headache with or without neurological deficits accompanied by multilocal caliber variation of the cerebral vessels. While RCVS usually shows a favorable outcome, in rare cases it can be associated with fatal complications such as hemorrhagic or ischemic brain lesions. Currently it remains unclear whether RCVS is linked to a second condition- Transient Global Amnesia (TGA). TGA is a benign neurological dysfunction that involves sudden temporary antero- and retrograde amnesia. The exact pathophysiological mechanisms involved in TGA and RCVS remain unclear but suggest common pathways as both can be triggered by similar factors. In this case report, we investigate the relationship of RCVS and TGA in a 65year-old female patient with a history of migraine and arterial hypertension. The initial admission to the ER was due to severe headache, hypertension and temporary memory loss, indicating TGA. Four days later, the patient returned to the clinic due to recurrent headache and hypertension.

An initial cMRI with angiography revealed no signs of an intracranial lesion or vasoocclusive pathology. A repeated $\mathrm{CCT}$ four days later showed a small left frontal subarachnoid hemorrhage. Complementary digital subtraction angiography recorded vessel diameter irregularities particularly along the distal vessel segments of the anterior circulation. Collectively, these findings indicated RCVS and the patient was admitted to the Stroke Unit for monitoring. MRI scans the following day confirmed the previously diagnosed subarachnoid hemorrhage in addition to bilateral hippocampal lesions as well as FLAIR hyperintensities frontoparietal. The former suited the TGA symptoms the patient experienced a few days earlier, the latter was to be classified in the context of RCVS. Repeated sonographic examinations could not verify progressive vasospasms and a follow up cMRI indicated nearly complete regression of the intracranial lesions. During hospitalization, the patient showed symptom relief and was discharged 10 days after admission.

We hypothesize that RCVS is the most likely cause for the symptoms leading to admission with TGA as a possible prodromal stage of RCVS. Whether the hypertensive pattern was the trigger for both symptom constellations remains unclear. In addition, the question of whether the hippocampal lesions are to be regarded as punctate ischemiae in the context of RCVS warrants further debate. 


\section{P05 COVID-19 Associated Stroke and Cerebral Endotheliitis, a Case Report}

\section{P. Stancu}

Hôpitaux Universitaire de Genève HUG, Switzerland

Context \& Objective: SARS-CoV2 has been associated with neurological complications, including an increased prevalence of stroke; one suggested pathophysiological mechanism is related to an endotheliopathy of cerebral small vessels. However, no evidence of such mechanism has been reported in vivo in patients with SARS-CoV2.

Case description: In this report, we present the case of a 81-year old patient who presented during a severe SARS-CoV2 infection multiple cerebral ischemic lesions, with contrast enhancement of bilateral vertebral right carotid and middle cerebral arteries.

He was admitted November 2020 in the University Hospital of Geneva for fever, dry cough and dyspnea. The diagnosis of SARS-CoV-2 was confirmed by RT-PCR. During the following days, the patient complained of gait instability and loss of coordination of his left hand. Neurological examination revealed a motoric and ataxic left hemisyndrome, with a NIHSS of 4. Brain MRI showed multiple small acute and subacute ischemic lesions, in particular in internal border zones between the deep and superficial arterial system of the middle cerebral artery, bilateral posterior cerebral arteries and bilateral cerebellar arteries. Neuroimaging findings show diffuse multi territorial and border zones ischemic lesions, despite the absence of vessels neck stenosis or evidence of decreased arterial pressure. Brain MRI with angiographic and intracranial vessel wall sequence show bilateral vertebral, right carotid and middle cerebral artery intracranial vessel wall contrast enhancement. The TOF angiography showed no stenosis that could be present in arteriosclerosis or localized vasculitis. Due to the morphology of the contrast enhancement, vasa vasorum enhancement was also judged unlikely.

Conclusions: Our findings are supportive of an inflammation of cerebral arteries consistent with endothelial dysfunction leading to stroke. Future neuroimaging studies is needed and should include a systematic evaluation of brain vessel wall imaging in COVID-19 patients with stroke.

P06 Recurrent Stroke in Patients with Atrial Fibrillation-A Possible Underestimation of Stroke Etiologies Other Than Cardioembolism?

\section{A. Zietz ${ }^{1}$, A. Polymeris ${ }^{1}$, F. Helfenstein ${ }^{2}$, L. Hert ${ }^{3}$, B. Wagner ${ }^{1}$, G. M. De Marchis ${ }^{1}$, H. Gensicke ${ }^{1}$, L. Bonati ${ }^{1}$, P. Lyrer ${ }^{1}$, S. Engelter ${ }^{1}$, N. Peters ${ }^{4}$}

1 Department of Neurology and Stroke Center, University Hospital and University of Basel, Switzerland

2 Clinical Trial Unit, Department of Clinical Research, University Hospital Basel, Switzerland

3 University and University Hospital of Basel, Switzerland

4 Stroke Center Hirslanden, Klinik Hirslanden Zurich and University Hospital of Basel, Switzerland

Introduction: Atrial fibrillation (AF) is a known and common cause for cardioembolic ischemic stroke, leading to oral anticoagulation $(\mathrm{OAC})$ for secondary stroke prevention. Nevertheless, stroke recurrence under OAC is seen in clinical practice, raising the question if competing stroke etiologies may be overlocked in this patient cohort. In the present study we are investigating the etiology of the index and recurrent stroke in patients with AF using the ASCOD, TOAST and Causative Classification System and their influence on the clinical course.

Methods: Observational study on stroke patients with AF enrolled in our prospective registry named Novel oral anticoagulants in stroke patients (NOACISP-Longterm). The TOAST classification is available for every ischemic stroke. In addition, we are using the 
ASCOD und Causative Classification system to describe the etiologies of the recurrent stroke and their respective index event. The primary endpoint is any of the following outcomes: ischemic recurrent stroke, intracerebral hemorrhage or death. The first event to occur will be considered as the endpoint. Our secondary endpoint will be ischemic recurrent stroke alone. Next to the descriptive analysis on clinical data and cardiovascular risk factors on patient with and without recurrent stroke, we will use Cox proportional hazard model to analyze how the time to first event varies according to the index stroke etiology. We will also run a cause-specific Cox proportional-hazard model and a competing risks Fine and Gray model if the data allows.

Results and Conclusions: In this ongoing study we will include approximately 1000 adult stroke patient with AF from the NOACISP stroke registry. In a first step we will investigate whether stroke etiologies differ regarding their association with one of our prespecified primary outcome measures. We will also present detailed demographic and clinical data of the subgroups with and without recurrent IS under secondary prevention with OAC and comparison of these two groups. In addition, we will classify index and recurrent stroke by various classification systems allowing detailed evalutation of etiology of recurrent stroke under OAC, an important clinical question potentially guiding individual treatment in these patients.

P07 The Role of Electrocardiographic Markers for Prediction of Atrial Fibrillation in Patients with Acute Ischemic Stroke: Data from the BIOSIGNAL Cohort Study

\section{Schütz}

Aim: We assessed pre-selected ECG parameters after acute ischemic stroke (AIS) and their value for predicting newly diagnosed atrial fibrillation (NDAF) after admission and up to 365 days after the index AIS.

Methods: We measured P-wave alterations on 12-lead ECG on admission in all patients without known AF consecutively enrolled in the University Hospital Zurich from October 2014-2017 as part of the prospective BIOSIGNAL cohort study (Biomarker signature of stroke etiology, NCT02274727). The outcome of interest was NDAF, identified by prolonged electrocardiographic monitoring within 1 year after the AIS. To address potential bias by missing values, models with and without multiple imputation were analyzed. 10-fold cross-validation was performed to better estimate the accuracy of the best predictive model. Results: NDAF was detected in $63(7.6 \%)$ of 830 eligible patients during a follow-up of 365 days. Out of the pre-selected ECG parameters only advanced interatrial block (aIAB) (OR 5.90, 95\%CI 3.35-10.38; $p<0.000)$ was independently associated with NDAF in univariable analysis and multivariable analysis with and without multiple imputation. Adding $\mathrm{aIAB}$ to the best multivariable regression model improved the discriminatory accuracy from an AUC of 0.73 (95\%CI $0.71-0.75)$ to 0.80 (95\%CI $0.78-0.82, p<0.01)$. The inter-rater reliability for aIAB was excellent with an $\alpha$ of 0.84 (95\%CI, $0.69-1.00$ ).

Conclusions: AiAB in 12-lead surface ECG during sinus rhythm is independently associated with NDAF in patients with AIS and can be used as a screening tool to improve diagnostic stroke work-up to search for AF.

\section{P08 The "Index Vein" Is a Highly Specific Sign for Migraine Aura in the Emergency Setting}

\section{A. Scutelnic ${ }^{1}$, V. Petroulia ${ }^{2}$, L. Schraml ${ }^{3}$, S. Jung ${ }^{1}$, M. Branca ${ }^{4}$, M. Beyeler ${ }^{1}$, U. Fischer ${ }^{5}$, R. Wiest ${ }^{2}$, N. Slavova ${ }^{6}$, C. Schankin ${ }^{1}$}

1 Inselspital-Universitätsspital Bern-Universitätsklinik für Neurologie, Switzerland

2 Inselspital-Universitätsspital Bern-Universitätsinstitut für Diagnostische und Interventionelle Neuroradiologie, Switzerland

3 Inselspital, Bern University Hospital and University of Bern, Switzerland 
6 Universitäts-Kinderspital beider Basel (UKBB), Switzerland

Objective: To assess the usefulness of the "index vein" (IV) for making the diagnosis of migraine aura in the emergency setting. We hypothesize that the IV occurs more often in migraine aura compared to epilepsy, ischemic stroke, and controls.

Methods: The IV was defined according to our previous work (Slavova et al. Neurology; 94(24): e2577-e2580): a single prominent vein in susceptibility weighted imaging draining the cortical area of the neurological deficit in the absence of diffusion abnormality and occlusion in angiography. 400 patients were included when they (i) presented at our emergency department with an acute neurological deficit, (ii) had a brain MRI within eight hours after the symptoms stopped, and (iii) had a discharge diagnosis of migraine aura, ischemic stroke, epilepsy or none of these (controls, $n=100$ per group). The primary hypothesis was that there is a different distribution of the IV in the four conditions. Further, we used multiple logistic regression to calculate a score (IV-score) consisting of IV and presence of visual and/or sensory symptoms and absence of loss of consciousness, paresis, coordination problems, hypertension and/or dyslipidemia.

Results: Compared to stroke $(n=2)$, epilepsy $(n=4)$ and controls $(n=1)$, the IV is more prevalent in migraine aura $(n=17, p<0.001)$. Although the sensitivity is low $(17 \%)$, we found the IV to be highly specific for migraine aura (specificity 97\%, 95\%CI 95-99). The IV has a positive predictive value for the diagnosis of migraine aura of 70\% (95\%CI 48-87), higher compared to stroke $(8 \%, 95 \% \mathrm{CI} 1-27)$, epilepsy $(16 \%, 95 \% \mathrm{CI} 4-37)$ and controls $(4 \%, 95 \% \mathrm{CI} 0.1-78)$. The IV-score has the ability to diagnose migraine with aura with a sensitivity of $89 \%$ (95\%CI 81.2-94.4) and specificity of 85\% (95\%CI 79.3-89.6).

Conclusions: When present, the IV serves as a good biomarker for migraine aura in the emergency setting. A simple to implement score, mainly based on history, may help to diagnose migraine with aura with high specificity and sensitivity.

P09 NEDA-3 Status and MAGNIMS Score in the First Two Years Predict Long-Term Disability: A Single-Centre Cohort Study

\author{
M. Pistor ${ }^{1}$, L. Diem ${ }^{1}$, H. Hammer ${ }^{1}$, C. Friedli ${ }^{1}$, N. Kamber ${ }^{1}$, F. Wagner ${ }^{2}$, R. Wiest ${ }^{2}$, \\ A. Chan ${ }^{1}$, A. Salmen ${ }^{1}$, R. Hoepner ${ }^{1}$ \\ 1 Inselspital—Universitätsspital Bern-Universitätsklinik für Neurologie, Switzerland \\ 2 Inselspital-Universitätsspital Bern-Universitätsinstitut für Diagnostische und Inter- \\ ventionelle Neuroradiologie, Switzerland
}

Introduction: Parameters to predict successful disability prevention in Multiple Sclerosis (MS) are still poorly validated. NEDA-3 status and MAGNIMS score might stratify for disability risk.

Objectives/Aims: To validate the first two-year individual NEDA-3 status and MAGNIMS scores as predictors for disability accumulation in an independent cohort of MS patients. Methods: A retrospective, single centre study was conducted (2005-2017). Inclusion criteria was relapsing-remitting MS (McDonald 2017). MAGNIMS score (new T2 lesions, relapses) and NEDA-3 status (Gadolinium pos./T2-lesions, relapses, disability progression) were assessed for the first two years after diagnosis. A composite cNEDA-3 (no NEDA-3 in both, NEDA-3 in one of two and NEDA-3 in two years) and a composite MAGNIMS score were formed (cMAGNIMS: low $=1$, medium $=2$, high $=3$ for each year, summed up for both years; range 2-6). Cox regression analysis was performed controlling for age, sex, baseline EDSS, treatment (no treatment, EMA (European Medicines Agency) first or second line) and treatment changes in first and second year. 
Results: 200 patients (61\% female, mean age 35.3 years (SD 11.4)) with a mean observation time of 79.43 months (SD 39.1) for those not reaching EDSS 6, and a baseline EDSS of 1.8 (SD 1.0) were analysed. Of these, $14 \%$ in 1 st and $12.5 \%$ in 2 nd year received no immunotherapy, $68 \%$ and $60 \%$ EMA first line and $18 \%$ and $27.5 \%$ second line therapies. NEDA-3 was reached by $39 \%$ of the patients for the 1 st and $63 \%$ for the 2 nd year. MAGNIMS score was "low" in 60\% in 1st and 76.5\% in 2nd year, "medium" 22\% and 18.5\% and "high" $18 \%$ and $5 \%$, respectively. Reaching NEDA-3 in one of the first two (Hazard Ratio (HR); 0.26 ; 95\%CI $0.09-0.73 ; p=0.01)$ or in both years $(0.12 ; 0.03-0.56 ; p=0.007)$ reduced the risk of reaching EDSS 4 in the given observation period. The HR for reaching EDSS 6 was $0.09(0.13-2.85 ; p=0.04)$ for NEDA-3 in both years and for one of the two $0.6(0.13-2.85$; $p=0.54)$. Compared to a cMAGNIMS of " 2 ", " 3 " had a HR of $1.7(0.40-7.62 ; p=0.45)$, " 4 " $3.4(0.98-11.59 ; p=0.05)$, “5” $7.4(1.25-43.58 ; p=0.03)$ and " 6 " $28.1(4.55-173.47 ; p<0.001)$ for reaching EDSS 4. Regarding reaching EDSS 6, only cMAGNIMS “6” was a significant predictor $(56.9 ; 4.00-810.47 ; p<0.001)$.

Conclusions: The two-year composites of NEDA-3 status and MAGNIMS scores were robust predictors of reaching disability milestones, highlighting their potential usefulness as surrogates.

\section{P10 Leptomeningeal Enhancement under Different Immunotherapies-A Monocentric Retrospective Cohort Study of 215 Patients}

\section{Friedli ${ }^{1}$, F. Wagner ${ }^{2}$, H. Hammer ${ }^{1}$, N. Kamber ${ }^{1}$, R. Wiest ${ }^{2}$, L. Diem ${ }^{1}$, A. Chan ${ }^{1}$, A. Salmen ${ }^{1}$, R. Hoepner ${ }^{1}$}

1 Inselspital-Universitätsspital Bern-Universitätsklinik für Neurologie, Switzerland

2 Inselspital-Universitätsspital Bern-Universitätsinstitut für Diagnostische und Interventionelle Neuroradiologie, Switzerland

Introduction: Leptomeningeal enhancement (LME) in multiple sclerosis (MS) patients consists mainly of meningeal B cell follicle-like structures that are linked to cortical lesions and subpial grey matter pathology. Therefore, LME is a new biomarker of disease activity. Objective: To evaluate the evolution of LME as a new imaging biomarker of disease activity under treatment with different immunotherapies.

Methods: Retrospective analysis of clinical and MRI data (either 1.5 or 3 T, follow-up exams always in the same scanner) since 2018 regarding LME of 215 MS patients treated with ocrelizumab, rituximab or fingolimod in a tertiary neurological center in Switzerland. Results: We evaluated 215 MS patients (mean age 43.3 years, range 18-79 years, 132/215 (61\%) female, 21 (9.7\%) with primary progressive (PP) and $194(90.3 \%)$ with relapsing remitting (RR) MS. 126/215 MS patients (58.6\%) received anti-CD20 therapies, (21/126 rituximab and 105/126 ocrelizumab) and 89/215 (41.4\%) patients received fingolimod. Each patient had one MRI before initiation and at least one (range 1-7) during treatment (mean 4 MRI per patient). Mean disease duration of all patients was 99.1 months (range 5-456 months), mean EDSS was 2.5 (range 0-7). Mean number of prior different immunotherapies was 1.1 (range 0-5). Mean observed treatment duration was 16 months with anti-CD20 therapies (range 1-56 months), and 50.3 months with fingolimod (range 5-115 months). Of the 126 patients treated with anti-CD20 therapies, 106 (84\%, 87 RR, 19 PP) patients had no LME, 11 (8.7\%, 9 RR, 2 PP) patients had persistent LME, whereas 9 (7.1\%, 8 RR, 1 PP) patients showed resolution of LME (all under ocrelizumab). Of the 89 RRMS patients treated with fingolimod, $84(94.4 \%)$ patients had no LME, $5(5.6 \%)$ patients had persistent LME, whereas none showed resolution of LME. Resolution of LME was significantly more frequent during anti-CD20 treatment as compared to fingolimod treatment $(p<0.05)$. Of those 9 patients with resolution of LME, 4 had no prior immunotherapies, the other 5 had 1-3 different prior immunotherapies. 
Conclusions: In our cohort, we observed LME resolution under treatment with ocrelizumab, but not under treatment with fingolimod or rituximab. As LME plays an important role in cerebral gray matter pathology, further investigations and extensions to higher field strengths (UHF MRI), correlation with clinical phenotypes and a comparison with other immunotherapies are needed.

\section{P11 Cortical Lesion Detection Using FLAWS in Multiple Sclerosis}

J. Müller ${ }^{1}$, F. La Rosa ${ }^{2}$, C. Tsagkas ${ }^{1}$, R. Rahmanzadeh ${ }^{1}$, J. Beaumont ${ }^{3}$, M. Barakovic ${ }^{1}$, P. Lu ${ }^{1}$, Ö. Yaldizli ${ }^{1}$, M. Weigel ${ }^{1}$, L. Kappos ${ }^{1}$, J. Kuhle ${ }^{1}$, M. Bach Cuadra ${ }^{2}$, G. Gambarota ${ }^{3}$, C. Granziera ${ }^{1}$

1 Translational Imaging in Neurology (ThINk) Basel and Neurology Clinic and Policlinic, Departments of Head, Spine and Neuromedicine, MS Center and Research Center for Clinical Neuroimmunology and Neuroscience (NC2NB), Department of Biomedical Engineering, Switzerland

2 Signal Processing Laboratory (LTS5), École Polytechnique Fédérale de Lausanne, Switzerland 3 Univ Rennes, Inserm, LTSI-UMR1099, France

Introduction: Cortical lesions (CL) are common in multiple sclerosis (MS), but their visualization is challenging on conventional MRI. Specialized T1-weighted sequences such as Magnetization Prepared 2 Rapid Acquisition Gradient Echoes (MP2RAGE) are able to improve cortical lesion detection. FLuid And White matter Suppression (FLAWS) provides an exquisite sensitivity to the grey matter signal and may facilitate cortical lesion identification.

Objective: To compare the manual detection rate of CL when using MP2RAGE or FLAWS. Methods: We included 30 relapsing-remitting MS patients who underwent MR imaging at 3T, including MP2RAGE and FLAWS. CL were segmented manually and classified as intracortical (ICL) or leukocortical lesions (LCL) by consensus of three trained raters on (i) MP2RAGE uniform/flat images and (ii) minimum FLAWS-contrast images that suppress both white matter and CSF signals (grey-matter-specific). Number and volumes of CL, ICL, LCL were compared using Wilcoxon signed rank tests. Comparison of proportions was performed using Friedman test. In an exploratory analysis, individual CL counts of the three raters were compared and interrater variability was quantified using Fleiss $\chi$.

Results: Cortical lesions were identified in 20/30 patients on MP2RAGE and 19/30 patients on FLAWS (66.6\% and $63.3 \%$, respectively). In total, 389 cortical lesions were identified, hereof 207 on MP2RAGE (43 ICL, 164 LCL, median CLs per patient 3, IQR 10) and 182 on FLAWS (42 ICL, $140 \mathrm{LCL}$, median CLs per patient 3.5, IQR 9; all $p>0.05$ ). In patients with CL, median lesion load of CL, LCL and ICL was comparable between the sequences (all $p>0.05)$. The same was true for the median volume of single CLs (MP2RAGE $47.73 \mathrm{~mL}$, IQR $43 \mathrm{~mL}$; FLAWS $54.91 \mathrm{~mL}$ IQR $46 \mathrm{~mL}, p>0.05$ ) and ICL (MP2RAGE $10.57 \mathrm{~mL}$, IQR $18 \mathrm{~mL}$; FLAWS $9.0 \mathrm{~mL}$, IQR $19 \mathrm{~mL}, p>0.05)$, but median size of LCL were segmented as larger on FLAWS (72.0 mL, IQR $70 \mathrm{~mL})$ than on MP2RAGE $(61.93 \mathrm{~mL}, \mathrm{IQR} 62 \mathrm{~mL} ; p=0.02)$. Interrater agreement of lesion counts was moderate for CL (MP2RAGE $\varkappa=0.436$; FLAWS $\varkappa=0.467$; both $p<0.001$ ), LCL (MP2RAGE $\varkappa=0.426$, FLAWS $\varkappa=0.463$; both $p<0.001$ ) and ICL on MP2RAGE $(\varkappa=0.514, p<0.001)$, but substantial for ICL on FLAWS $(\varkappa=0.661$, $p<0.001)$.

Conclusions: Size of identified cortical lesions was larger on FLAWS than on MP2RAGE while overall detection rate of cortical lesions was similar when performed on MP2RAGE or FLAWS. 
P12 Choroid Plexus Volume in Multiple Sclerosis vs. Neuromyelitis Optica Spectrum Disorder

J. Müller ${ }^{1}$, T. Sinnecker ${ }^{1}$, M.J. Wendebourg ${ }^{1}$, R. Schläger ${ }^{1}$, J. Kuhle ${ }^{1}$, S. Schädelin ${ }^{1}$, P. Benkert ${ }^{1}$, T. Derfuss ${ }^{1}$, P. Cattin ${ }^{2}$, C. Jud ${ }^{2}$, F. Spiess ${ }^{2}$, M. Amann ${ }^{3}$, T. Lincke ${ }^{4}$, M. Barakovic ${ }^{5}$, A. Cagol ${ }^{5}$, C. Tsagkas ${ }^{1}$, A. Pröbstel ${ }^{1}$, S. Reimann ${ }^{1}$, S. Asseyer ${ }^{6}$, A. Duchow ${ }^{6}$, A. Brandt ${ }^{6}$, K. Ruprecht ${ }^{7}$, N. Hadjikhani ${ }^{8}$, S. Fukumoto ${ }^{9}$, M. Watanabe ${ }^{9}$, K. Masaki ${ }^{9}$, T. Matsushita ${ }^{9}$, N. Isobe ${ }^{9}$, J. Kira ${ }^{9}$, L. Kappos ${ }^{1}$, J. Würfel ${ }^{3}$, C. Granziera ${ }^{5}$, F. Paul ${ }^{6}$, Ö. Yaldizli ${ }^{1}$

1 Neurology Clinic and Policlinic, Departments of Head, Spine and Neuromedicine, MS Center and Research Center for Clinical Neuroimmunology and Neuroscience Basel (RC2NB), Clinical Research and Biomedical Engineering, University Hospital Basel and University, Switzerland

2 Department of Biomedical Engineering, University of Basel, Switzerland

3 Medical Imaging Analysis Center AG, Switzerland

4 Section of Neuroradiology, Clinic for Radiology \& Nuclear Medicine, University Hospital Basel and University of Basel, Switzerland

5 Translational Imaging in Neurology (ThINk) Basel, Department of Biomedical Engineering, University Hospital Basel and University of Basel, Switzerland

6 NeuroCure Clinical Research Center and Experimental and Clinical Research Center, Max Delbrueck Center for Molecular Medicine and Charité-Universitätsmedizin Berlin, Germany

7 Department of Neurology, Charité-Universitätsmedizin Berlin, corporate member of Freie Universität Berlin and Humboldt-Universität zu Berlin, Germany

8 Martinos Center for Biomedical Imaging, Massachusetts General Hospital and Harvard Medical School, USA

9 Department of Neurology, Neurological Institute, Graduate School of Medical Sciences, Kyushu University, Japan

Background: The choroid plexus $(\mathrm{CP})$ has been shown to play a crucial role in central nervous system inflammation. Previous studies have shown increased $\mathrm{CP}$ volume in multiple sclerosis (MS) compared to healthy control subjects (HCs). However, it is not clarified whether the CP is also enlarged in neuromyelitis optica spectrum disorder (NMOSD). So, the aim of this study was to compare the CP volume in MS and NMOSD.

Methods: We studied 180 MS patients from the University Hospital Basel Kyushu Fukuoka, 98 NMOSD patients from the University Hospitals Basel, Berlin and the Kyushu Fukuoka, 39 patients with migraine from the University Hospital Lausanne and 91 HCs from the University Hospitals Basel, Lausanne and the Kyushu Fukuoka. The CP of the lateral ventricles was segmented fully automatically on T1-weighted MRI sequences using a deep learning algorithm (Multi-dimensional Gated Recurrent Units). Uni- and multivariable general linear models were applied to investigate associations of the $\mathrm{CP}$ volume with clinical, MRI and laboratory measures using age, gender, disease duration, disability status, relapse in the previous year and number of T2-weighted lesions as covariates. A multivariable regression model was used to differentiate MS from NMOSD using the CP volume as independent covariate.

Results: The CP was larger in MS than NMOSD (median volume $1690 \mu \mathrm{L}$, interquartal range: 648 vs. $1403 \mu \mathrm{L}$, interquartal range: $510 ; p<0.001 ; \mathrm{d}=0.78$ ). Moreover, the $\mathrm{CP}$ was larger in MS than in migraine patients (median $1480 \mu \mathrm{L}$, interquartal range: 459; $p=0.007$; $\mathrm{d}=0.51$ ) and in HCs (median $1562 \mu \mathrm{L}$, interquartal range: 627; $p<0.001 ; \mathrm{d}=0.4$ ). These differences were also statistically significant after adjusting for age, gender and intracranial volume (all $p<0.05)$. In contrast, there was no difference in the $\mathrm{CP}$ volume between neuromyelitis optica spectrum disorder, migraine and healthy individuals. Patients with secondary progressive MS, higher T2-weighted lesion number, higher efficacious disease 
modifying therapy (DMT) and longer DMT duration had larger CP than patients with relapsing-remitting MS, lower T2-weighted lesion number, less efficacious DMT or shorter DMT duration. Every $100 \mu \mathrm{L}$ larger CP increased the risk of having MS instead of NMOSD by $21 \%$ in a multivariable logistic regression model.

Conclusions: Our data support an involvement of the $\mathrm{CP}$ in MS and may provide clues to better understand the respective pathogenesis.

P13 Case Report: Meningovascular Neuroborreliosis with Multiple Strokes and Persistent Headache

\section{Achtnich, M. Fett}

Kantonsspital St. Gallen, Switzerland

Background: Meningovasculitis with associated strokes is a rare form of neuroborreliosis. The clinical presentation with long lasting headache, posterior circulation stroke and associated basal meningitis is typical. Pathoanatomically ischemic stroke is explained by paravascular vasculitic angiopathy or local immunoreaction. We present the case of a 21-year-old woman who suffered from multiple ischemic strokes in combination with basal meningitis associated to Lyme borreliosis.

Case Report: A 21-year-old female presented to the emergency department with left sided hemiparesis of sudden onset (NIHSS 4). Since 3 months she suffered from therapy-resistant occipital headache and subfebrile temperatures. Brain-MRI revealed acute ischemic stroke in the right thalamus and subacute ischemic strokes in the cerebellum. No large vessel abnormalities were detected. Acute treatment with intravenous thrombolysis with rtPA was performed. Cerebrospinal fluid (CSF) analysis the next day showed granulocytic pleocytosis ( 96 cells $/ \mathrm{mcl}$ ) and disturbance of blood-brain barrier. Further investigations showed lymphoplasmacellular pleocytosis in the CSF. Follow-up MRI showed contrastenhancement of basal meninges suggesting basal meningitis. Empiric anti-infective treatment with Ceftriaxone and Acyclovir was started.

Two days after initiation of Ceftriaxone, headache stopped. Acyclovir was stopped (negative HSV and VZV-PCRs). Positive Borrelia Burgdorferi Liquor-Serum Indices and Serologies and positive B. Burgdorferi Immunoblot confirmed neuroborreliosis. CXCL13 was highly positive $(745 \mathrm{pg} / \mathrm{mL}(<250 \mathrm{pg} / \mathrm{mL}))$. Further diagnostic showed no evidence of other aetiologies (paraneoplastic, lymphoma, infectious, rheumatic disease or primary CNS-vasculitis).

Results: Ceftriaxone was continued for 21 days. Clinical follow-up 2 Month later showed a good outcome with only slight persisting left sided paresthesias and no evidence of basal meningitis on MRI. CSF cell count was still slightly elevated $(17 \mathrm{cells} / \mathrm{mcl})$ with minor disturbance of the blood-brain-barrier. CXCL13 was no longer detectable in the CSF.

Conclusions: The combination of long lasting headache and strokes in young patients should let us think about rare aetiologies of stroke including neuroborreliosis with associated meningovasculitis. Positiv Liquor-Serum indices for B. Burgdorferi and an inflammatory CSF syndrome can lead to the diagnosis. Early begin of anti-infective therapy is associated with good clinical outcome.

P14 Vaccination Prior to Treatment with Ocrelizumab: A Retrospective Observational Study

V. Epple, C. Berger, S. Schaedelin, P. Benkert, L. Kappos, K. Leuzinger, H. Hirsch, J. Kuhle, M. Mehling

Universitätsspital Basel, Switzerland

Background: Humoral vaccine responses are attenuated in persons with MS (pwMS) treated with ocrelizumab (OCR). Accordingly, it is recommended to complete vaccinations 
before starting treatment with OCR to ensure best possible vaccine response. In Switzerland, a waiting period of 6 weeks after vaccination is recommended before starting treatment with OCR. Particularly in pwMS with high disease activity, this waiting period is not always feasible.

Objectives: To assess whether an interval of less than 4 weeks between vaccination and start of treatment with OCR is associated with an attenuated humoral vaccine response in pwMS.

Methods: We retrospectively analyzed clinical data and serum of pwMS that are followed in the Swiss MS Cohort at the University Hospital Basel. PwMS having received either a pneumococcal conjugate vaccine and/or a booster dose of tetanus- and diphtheriatoxin-containing vaccine before starting a treatment with OCR were recruited to the study. Antibody levels before and after vaccination were compared among pwMS who received OCR either less than or at least 4 weeks after vaccination.

Results: Antibody levels were analyzed in 58 pwMS (mean age 44 years, 36 female). 46 pwMS (79\%) had a relapsing and $12(21 \%)$ a primary progressive MS. Median disease duration was 5.5 years and median EDSS was 3.0. 21 patients (36\%) were treatment naïve and 37 patients (64\%) were pretreated with another DMT, before starting OCR. All patients received a pneumococcal conjugate vaccine and 41 patients a tetanus- and diphtheria-toxin-containing vaccine. 18 pwMS received OCR less than 4 weeks after vaccination (median time interval: 18 days) while 29 pwMS received OCR at least 4 weeks after vaccination (median time interval: 44 days). 11 pwMS had B-cell depleting therapy before vaccination. 3-15 months after vaccination, pwMS with an interval of less than 4 weeks between vaccination and first dose of OCR achieved comparable median anti-S. pneumoniae $\operatorname{IgG}$ [246.65 $\mu \mathrm{g} / \mathrm{mL}$ ], anti-tetanus-toxin IgG [2.83 IU/mL] and anti-diphtheriatoxin $\mathrm{IgG}[0.48 \mathrm{IU} / \mathrm{mL}]$ levels as pwMS with an interval of at least 4 weeks $[191.70 \mu \mathrm{g} / \mathrm{mL}$, $2.62 \mathrm{IU} / \mathrm{mL}, 0.46 \mathrm{IU} / \mathrm{mL}]$.

Conclusions: In our study, no relevant attenuation of the humoral vaccine response was observed by shortening the interval between vaccination and initiation of therapy with OCR to less than 4 weeks. Considering the limitations of a small observational study, further investigations to confirm these findings are needed.

P15 Ganglion Cell-Inner Plexiform Layer Is a Marker of Brain Neuronal Loss in Multiple Sclerosis
A. Cagol 1, N. Cerda-Fuertes ${ }^{1}$, M. Stoessel ${ }^{1}$, M. Barakovic ${ }^{1}$, R. Rahmanzadeh ${ }^{1}$, R. Galbusera ${ }^{1}$, M. D' Souza ${ }^{1}$, J. Wuerfel ${ }^{2}$, T. Sprenger ${ }^{3}$, L. Kappos ${ }^{1}$, Y. Naegelin ${ }^{1}$, J. Kuhle ${ }^{1}$, C. Granziera ${ }^{1}$, A. Papadopoulou ${ }^{1}$
1 University of Basel/University Hospital Basel, Switzerland
2 University Hospital Basel and University of Basel/Medical Image Analysis Center Basel AG, Basel, Switzerland
3 DKD Helios Klinik Wiesbaden, Germany

Background: The ganglion cell-inner plexiform layer (GCIPL), measured by optical coherence tomography (OCT), may be a marker of neuronal loss in multiple sclerosis (MS). Prior studies showed associations of GCIPL with brain volume measures (1-2), but its relationship with regional gray (GM) and white matter (WM) damage was not thoroughly investigated.

Objectives: To assess the relationship of GCIPL with global and regional GM and WM measures of tissue loss in MS and to study its relation to disability.

Methods: This cross-sectional study included $90 \mathrm{MS}$ patients (63\% female; age: $49.2 \pm 11.2$ years(y); median disease duration: 18 (10-24.5) y; median EDSS 3.0 (1.5-3.5); 83\% relapsing-remitting MS) and 48 healthy controls (HC; 65\% female; mean: $51.1 \pm 13.1 \mathrm{y})$. All subjects received OCT and 3T brain MRI on the same day. Eyes with prior optic neuritis were excluded. GM 
and WM volumes were obtained with FreeSurfer. Their associations with GCIPL volume were investigated with general linear models adjusting for age, sex, T2 lesion volume and total intracranial volume; for cortical thickness (CTh) a vertex-wise analysis was conducted. Results were adjusted for multiple comparisons. A hierarchical regression model including demographics (age and sex) and MRI measures (cortical GM, deep GM and WM volumes) was used to assess the association of GCIPL volume with EDSS (log).

Results: Patients showed lower GCIPL volume $(p<0.001)$, as well as lower volumes of cortical GM, deep GM and WM than HC (all $p<0.001$ ).

In patients, GCIPL volume was associated with volumes of cortical GM $(\beta=0.257, p=0.009)$ and deep GM $(\beta=0.222, p=0.012)$, but not with total WM volume $(p=0.515)$. Regional analyses revealed significant associations of GCIPL with the thickness of multiple cortical clusters in the temporal, parietal, and occipital lobes, bilaterally (all $p<0.03$ ); no associations with regional WM volumes were present. In HC we found no associations between GCIPL volume and MRI measures. Regarding disability, GCIPL was an independent predictor of the EDSS $(\beta=-0.410, p<0.001)$ in the multivariate model; its addition to the model including demographic and MRI measures resulted in an R2 increase of 0.13 (0.51 vs. 0.38). Conclusions: We observed associations of GCIPL specifically with GM- (rather than WM-) measures, also outside of the afferent visual pathway, and with the patients' disability status. These findings support the role of GCIPL as marker of global neuronal loss in MS.

P16 Hypogammaglobulinemia: A Possible Cause of Multiple Sclerosis-Related Fatigue?

\section{Diem ${ }^{1}$, M. Evangelopoulos ${ }^{2}$, D. Karathanasis ${ }^{2}$, V. Natsis ${ }^{2}$, N. Kamber ${ }^{1}$, H. Hammer ${ }^{1}$, C. Friedli ${ }^{1}$, A. Chan ${ }^{1}$, A. Salmen ${ }^{1}$, R. Hoepner ${ }^{1}$}

1 Inselspital-Universitätsspital Bern-Universitätsklinik für Neurologie, Switzerland

2 Eginition University Hospital, National and Kapodistrian University of Athens, Greek

Introduction: Fatigue is one of the most disabling symptoms of multiple sclerosis (MS). However, effective treatment strategies for MS-fatigue are still missing. Reduced IgG serum levels affect approximately 8-25\% of the people with MS with unknown consequences. Objective: Retrospective analysis to investigate the association of MS-fatigue and reduced IgG serum levels (definition: $<7.0 \mathrm{~g} / \mathrm{L}$ ).

Methods: Patients with available Fatigue Scala for Motor and Cognitive Function (FSMC) data treated at Eginition University Hospital Athens or at the University Hospital Bern were identified by medical chart review leading to a cohort of 134 patients. Comparative statistics including Mann Whitney (MWT), ANOVA test, Chi2 Test as well as multivariate regression models were performed.

Results: $97 / 134(72.4 \%)$ reported at least a mild global and 89/134 (66.4\%) a mild cognitive and $97 / 134(72.4 \%)$ a mild motor fatigue as defined by FSMC. Compared to MS-patients with normal serum IgG concentrations, MS-patients with reduced IgG levels reported significantly more frequently fatigue. In those affected by fatigue, mean severity of fatigue was significantly higher for IgG-deficient patients (mean $(95 \%$ confidence interval $(95 \% \mathrm{CI})$ 67.6 (62.9-72.4) vs. 55.7 (50.8-58.7), MWT $p=0.005)$.

Conclusions: In this retrospective study, we found an association between reduced serum IgG levels and fatigue in people with MS. The reason for fatigue in patients with immunodeficiency might be an association with more frequent infections, which cause fatigue. Longitudinal studies shall assess the role of IgG levels in MS-related fatigue under the light of a potentially treatable immunological cause of MS-fatigue.

P17 Ocrelizumab-Related Neutropenia: Effects of Age, Sex and Bodyweight Using the FDA Adverse Event Reporting System (FAERS)

H. Hammer, N. Kamber, M. Pistor, L. Diem, C. Friedl, A. Chan, R. Hoepner, A. Salmen 
Inselspital—Universitätsspital Bern—Universitätsklinik für Neurologie, Switzerland

Introduction: Safety and efficacy of Ocrelizumab (OCR) have been evaluated in randomized controlled studies with infusion-associated reactions being the most prevalent adverse event. Whereas slight decreases in neutrophil counts occurred in $13-15 \%$ of patients during the pivotal trials, grade 4 neutropenia was observed in up to $1 \%$ of patients and can develop as late-onset neutropenia as already reported for Rituximab. Potential risk factors for OCR-related neutropenia are still unclear.

Aims: To investigate Neutropenia in OCR treated MS patients.

Methods: Safety data was retrieved from the open source data registry provided by the FDA Adverse Event Reporting System (FAERS, date of download 12 April 2021). Only data with OCR as the single suspected product used were included. Multivariable logistic regression analysis was conducted to assess the association of MS disease course, age, sex and bodyweight with risk of neutropenia in OCR-treated MS patients.

Results: We here present data of U.S. Food and Drug Administration (FDA) Adverse Event Reporting Systems (FAERS), a post-marketing, open access pharmacovigilance platform to identify risk factors of OCR-related Neutropenia. Data on age, sex, bodyweight, and MS disease course were present in 3177 of 15,313 reports in total. Neutropenia occurred in 25/3177 of these cases. The outcome was rated as follows: hospitalized $17 / 25$, life threatening $2 / 25$, and other outcome $6 / 25$. No deaths were reported. Multivariable logistic regression demonstrated that MS disease course was not associated, whereas younger age $(0.909,95 \%$ CI $0.875-0.944, p<0.05)$, lower bodyweight $(0.961,95 \%$ CI $0.935-0.988$, $p<0.05)$ and female sex $(0.356,95 \% \mathrm{CI} 0.145-0.875, p<0.05)$ were significant predictors of OCR-related neutropenia (Nagelkerkes R2 $=0.17, n=3177$ ).

Conclusions: We demonstrated that female sex, younger age and lower bodyweight are predictors of OCR- related neutropenia. Limitations of our study are associated with the design of the open data registry with several missing values and only a minimal clinical data set available as well as bias inherent to spontaneous reporting systems. The latter may account for both the overall lower rate of events as compared to the controlled trials and the higher rate of more severe outcomes.

P18 Lowering Blood Cholesterol Does Not Affect Inflammation in a Murine Model of Multiple Sclerosis

\section{S. Vigne ${ }^{1}$, D. Duc ${ }^{1}$, J. Rebeaud ${ }^{1}$, B. Peter ${ }^{1}$, F. Ruiz ${ }^{1}$, Y. Yersin ${ }^{1}$, V. Bressoud ${ }^{1}$, T. Collet ${ }^{2}$, C. Pot ${ }^{1}$}

1 Lausanne University Hospital (CHUV) and University of Lausanne (UNIL), Switzerland

2 Geneva University Hospitals (HUG), Switzerland

Context and Objectives: Multiple sclerosis (MS) is a chronic disabling disease of the central nervous system (CNS) commonly affecting young adults (1). Metabolic syndrome (MetS) including dyslipidemia has been associated with MS onset and disease progression (2). Previous studies reported an association between elevated levels of total circulating cholesterol and their carrier lipoproteins with a worse disease outcome (3-5). However, the role of lowering cholesterol levels during MS is controversial: while statin treatments were initially proposed to be beneficial, the impact of circulating blood cholesterol level during the disease remains unclear as for today and studies have shown contradictory results also on different subset of MS (6-8). Thus, the role of cholesterol metabolism during MS is largely debated.

The objective of our study was to assess the importance of circulating cholesterol on the murine model of MS, the experimental autoimmune encephalomyelitis (EAE) disease.

Methods: We assessed the importance of circulating cholesterol using a MS murine model, the experimental autoimmune encephalomyelitis (EAE) and two different strategies: (1) a mouse model of familial hypercholesterolemia where genetic deletion of LDLr (coding for 
the low-density lipoprotein receptor) causes a significant increase of plasma cholesterol concentrations and (2) the use of a monoclonal PCSK9 inhibitor which reduces LDLr degradation and consequently lowers plasma cholesterol concentrations.

Results: We show that circulating hypercholesterolemia induced by LDLr deficiency does not affect development of EAE disease independently of the mice gender. In addition, we observed that using a human monoclonal anti-PCSK9 neutralizing antibodies alirocumab, significantly decreased the circulating cholesterol level in WT mice but did not influence EAE clinical course nor modulate the adaptive immune responses during the development of CNS autoimmunity.

Conclusions: Our study suggests that circulating cholesterol does not impact the development of EAE disease, and, therefore, does not support the hypothesis that statin beneficial effects can be attributed to its effect on lowering serum cholesterol levels and its consequent improved hyperlipidemia, which is known to be comorbidity in MS.

\title{
P19 Prognostication of Confirmed Disease Progression in Patients with Primary Progressive MS (PPMS) by Serum Neurofilament Light Chain Levels and Multimodal Evoked Potentials
}

\author{
M. Hardmeier ${ }^{1}$, R. Schlaeger ${ }^{1}$, A. Lascano ${ }^{2}$, L. Toffolet $^{3}$, C. Schindler ${ }^{4}$, C. Gobbi ${ }^{3}$, \\ P. Lalive ${ }^{2}$, D. Leppert ${ }^{1}$, L. Kappos ${ }^{1}$, P. Fuhr ${ }^{1}$, J. Kuhle ${ }^{1}$ \\ 1 University Hospital Basel/University of Basel, Switzerland \\ 2 Geneva University Hospitals (HUG), Switzerland \\ 3 Neurocenter of southern Switzerland, Regional Hospital Lugano (EOC), Switzerland \\ 4 University of Basel/Swiss TPH, Switzerland
}

Introduction: In clinical trials on primary progressive MS (PPMS) $35-45 \%$ of untreated subjects experience confirmed disease progression (CDP) against which a treatment effect is to be measured. Prognostic biomarkers may help to increase the event rate.

Objectives

To determine the prognostic value of serum neurofilament light chain (sNfL) levels and evoked potential (EP) scores for CDP during 24 months of follow-up in PPMS.

Methods: 35 patients with PPMS (16 women; mean age: 52.5 years; median EDSS: 4.0, range 2.0-6.5) had upper and lower limb motor and sensory EP and sNfL measurements at baseline, and at least annual EDSS assessments by certified raters. Three subjects were on CD20-depleting therapy at baseline, 20 started on study. CDP was defined as increase in EDSS by 1.0 (by 0.5 , if baseline EDSS > 5.5) confirmed at next follow-up.

Sensory and motor EP latencies were height-adjusted and z-transformed to calculate a modified quantitative EP score (mqEPS) and a quantitative motor EP score (qMEP). sNfL levels were measured with the NF-light ${ }^{\circledR}$ assay and transformed to age-adjusted z-scores using normative data of healthy controls.

Uni- and bivariable logistic regression and receiver operating characteristic curve (ROC) analyses were performed $\left({ }^{*} p<0.05,{ }^{* *} p<0.01,{ }^{* * *} p<0.001\right)$.

Results: Median (interquartile range) sNfL level was $13.2 \mathrm{pg} / \mathrm{mL}$ (8.9-17.2), sNfL z-scores 1.1 (0.4-2.6), mqEPS, 3.0 (1.9-5.1), qMEP 2.5 (1.1-5.3). Compared to stable patients, subjects with CDP $(n=13 ; 37 \%)$ were younger ( 46.8 vs. $\left.55.9^{*}\right)$ had higher sNfL z-scores (2.6 vs. $\left.0.7^{* * *}\right)$, mqEPS (5.7 vs. $2.5^{* *}$ ) and qMEP (5.7 vs. $1.5^{* *}$ ).

CDP was more likely with higher sNfL z-scores (OR per unit-increase: $3.8,95 \% \mathrm{CI}$ : $1.6-9.1^{* *}$ ), mqEPS (OR: 1.9, 95\%CI: 1.1-3.1*) and qMEP (OR: 1.7, 95\%CI: 1.1-2.6*). In a bivariable model, sNfL z-scores and qMEP independently predicted CDP (OR: 3.0, 95\%CI: 1.7-7.8* and OR: $1.8,95 \% \mathrm{CI}$ : $1.0-3.1^{*}$, respectively).

Cutoffs at maximized sums of sensitivity and specificity derived from the ROCs of sNfL z-score, mqEPS and qMEP, provided the same sensitivity (77\%) in predicting CDP, at 
specificities of $82 \%, 86 \%, 91 \%$ and AUCs of $0.85,0.84$ and 0.84 , respectively. sNFL z-score and qMEP combined yielded highest sensitivity (85\%), specificity (95\%) and AUC (0.91). Conclusions: EP scores and sNfL levels are independent predictors of disease progression in PPMS. In clinical trials, their combined use may allow for selection of patients with high probability of progression.

\section{P20 Effects of Receptive Music Therapy with a Monochord in Multiple Sclerosis} (MuTiMS)_A Randomized Controlled Study

\section{J. Kuhlmann ${ }^{1}$, K.A. Ebner ${ }^{1}$, N. Cerdá Fuertes ${ }^{1}$, K. Parmar ${ }^{2}$, F. Helfenstein ${ }^{1}$, A. Zimmer ${ }^{1}$, A. Papadopoulou ${ }^{1}$}

1 Universitätsspital Basel, Switzerland

2 Rehabilitationsklinik Rheinfelden, Switzerland

Background: Music Therapy (MT) is a branch of art therapy, which could improve anxiety and depressive symptoms in patients with neurological disorders. In multiple sclerosis (MS), previously published studies with MT were uncontrolled or too small, to detect an evident therapeutic effect. Moreover, people with MS are usually exposed to MT in rehabilitation clinics rather than outpatient MS centers.

Aim: With our randomized, controlled trial we aim at investigating if ambulatory MT can improve symptoms of anxiety in patients with MS (primary end-point). Our secondary end-points are improvements in depressive symptoms, health-related quality of life, stress, fatigue, cognition and pain thresholds.

Methods: According to our power calculation, we will include sixty patients with MS in the study (both relapsing-remitting and progressive MS allowed). Ambulatory patients are being enrolled in our MS center and randomly assigned 1:1 to two groups: the MT group and the control group. Participants in the MT group have 6 weekly sessions of MT, with laying on top of the instrument "monochord" and relaxing to its music, played by the music therapist. In the control group, participants have the same amount of sessions with laying down on top of the monochord, but without music being played. A rater blinded to the assignment assesses changes in anxiety symptoms (Hospital Anxiety and Depression Scale, HADS-A) and the other secondary measures with questionnaires. Moreover, the same rater assesses the patients' pain thresholds (thermal-, mechanical- and pressure pain) using quantitative sensory testing. At end of study, the two groups will be compared for the primary- and secondary end-points using linear mixed models, with adjustment for relevant confounders.

Preliminary results: Up to date, 10 patients were randomized and 7 completed the study. The baseline characteristics of the randomized patients were: age (mean \pm SD): $54.4 \pm 14$ years, sex: 10/10 women, EDSS (median, min-max): 2.5 (1.5 to 6.5), relapsing-remitting MS (RRMS): 6/10, progredient MS (SPMS, PPMS): 4/10. Recruitment is currently ongoing. Conclusions: This controlled study can provide evidence to promote ambulatory MT in MS centers and expand the non-pharmacological therapeutic options for people with MS. Moreover, it can shed light to the mechanisms of action of MT and detect the subgroup of patients that may profit at most from such an intervention.

\section{P21 A Case of SARS-CoV-2-Associated Acute Disseminated Encephalomyelitis}

\section{N. Lohaus ${ }^{1}$, I. Reichen ${ }^{1}$, R. Reimann ${ }^{2}$, I. Abela ${ }^{3}$, I. Jelcic ${ }^{1}$}

1 Department of Neurology, University Hospital and University of Zurich, Switzerland 2 Institute of Neuropathology, University Hospital and University of Zurich, Switzerland

3 Department of Infectious Diseases and Hospital Epidemiology, University Hospital and University of Zurich, Switzerland 
Background and aim: Severe acute respiratory syndrome coronavirus type 2 (SARS-CoV-2) is currently spreading globally causing coronavirus disease 2019 (COVID-19) pandemic. Aside from fever and respiratory symptoms, distinct neurological disorders have been associated with SARS-CoV-2 infection, e.g., metabolic/hypoxic encephalopathy, stroke, seizure and Guillaine-Barré syndrome. Association is in most cases established through co-incidence, i.e., association in time, but proof by cerebrospinal fluid (CSF) diagnostics is scarce. We here describe a case of central nervous system (CNS) autoimmune disease most probably caused by asymptomatic SARS-CoV-2 infection.

Material and methods: Single case presentation.

Results: A 28-year-old female was admitted to our center with her first generalized seizure. In the three weeks before she had suffered from attentional deficits and constant headache that were worse in the morning or when bending her head down. MRI showed a left frontal mass with perifocal edema and patchy contrast enhancement, raising the suspicion of cerebral lymphoma. CSF analysis revealed mononuclear pleocytosis of 14 cells $/ \mu \mathrm{L}$ without cytological signs of malignancy. Immunophenotyping of CSF and blood showed no evidence of lymphoma. Albumin ratio, CSF total protein, Reibergram, glucose ratio and CSF lactate were normal, oligoclonal bands were negative. Acute disseminated encephalomyelitis (ADEM) was suspected and finally confirmed by histopathological results from brain biopsy. Re-questioning of the patient revealed no history of recent symptomatic infections or vaccinations, but a history of two high-risk contacts to COVID-19 patients, one four weeks and another five months ahead of the seizure. Subsequently, serological testing detected CSF and serum antibodies against four SARS-CoV-2-derived antigens and intrathecal production of IgG against two SARS-CoV-2 antigens using an in-house developed bead-based assay (ABCORA 2.0). SARS-CoV-2 PCR analysis of blood and CSF was negative. MRZ reaction and serum MOG- and AQP4-specific antibodies were negative. The symptoms improved rapidly after high-dose methylprednisolone therapy with subsequent tapering.

Conclusions: The findings suggest SARS-CoV-2 infection without respiratory symptoms as the cause of ADEM in this case. Clinicians should be aware of atypical SARS-CoV-2 infection as a potential trigger of post-infectious neurological autoimmune disease.

\title{
P22 A Case of Posttransplant Anti-GABA-a Receptor Antibody-Associated
} Autoimmune Encephalitis

\author{
C. Togni ${ }^{1}$, M. Schubring-Giese ${ }^{2}$, L. Imbach ${ }^{3}$, I. Jelcic ${ }^{1}$ \\ 1 Universitäts Spital Zürich, Switzerland \\ 2 Zuger Kantonsspital, Switzerland \\ 3 Klinik Lengg, Switzerland
}

Background: First described in 2014, autoimmune encephalitis associated with autoantibodies against $\gamma$-aminobutyric acid receptor type A (GABA-A-R) is a rare autoimmune encephalitis characterized by new-onset epilepsy, cognitive and behavioral changes and multifocal cortico-subcortical lesions on MRI, sometimes occurring as a parainfectious or paraneoplastic phenomenon [1,2]. Here, we report a case of anti-GABA-A-R encephalitis following double autologous hematopoietic stem cell transplantation (aHSCT) for multiple myeloma (MM).

Materials and Methods: Single case report.

Results: A 58-years old patient was admitted to the hospital due to rapidly progressive neurological deterioration, including altered mental status, memory deficits, hypomanic behavior, gait instability, left-sided hemiparesis and new-onset focal epileptic seizures culminating in therapy-refractory focal status epilepticus and myoclonia continua. 15 and 18 months prior to admission, he had undergone double aHSCT for MM and has since been on maintenance therapy with lenalidomide. Sequential magnetic resonance imaging (MRI) 
revealed multifocal, supratentorial, cortico-subcortical, edematous, non-contrast enhancing T2w/FLAIR-hyperintense lesions varying in size and localization within few weeks. As serum tested positive for anti-GABA-A-R antibodies, a diagnosis of posttransplant anti-GABA-A-R encephalitis was made. Upon aggressive immunosuppressive therapy, including plasmapheresis, repeated high-dose corticosteroids with slow tapering and rituximab, as well as combined anticonvulsive therapy, he experienced near-complete clinical recovery with total regression of all MRI changes and has since remained seizure-free.

Conclusions: Here, we provide the second case of anti-GAB-A-R encephalitis following aHSCT described in the literature [2]. Posttransplant autoimmune disease is thought to arise from partially aberrant immune reconstitution, including imbalanced B- and T-cell recovery and breakdown of self-tolerance, and may occur up to five years after HSCT [3]. This case highlights, that the spectrum of posttransplant autoimmune diseases includes neurological disorders, among them autoimmune encephalitis [4]. Finally, our case provides further evidence for a favorable outcome in patients with anti-GABA-A-R encephalitis receiving rituximab.

\section{P23 MRZ Reaction in Mog Antibody-Associated Autoimmune Disease and Multiple} Sclerosis: A Single-Center Experience

\section{Reichen ${ }^{1}$, B. Vlad ${ }^{1}$, D. Lekaditi ${ }^{1}$, C. Heuer ${ }^{1}$, A. Eisele ${ }^{1}$, M. Ziegler ${ }^{1}$, M. Reindl ${ }^{2}$, A. Lutterotti ${ }^{1}$, A. Regeniter ${ }^{3}$, I. Jelcic ${ }^{1}$}

1 Department of Neurology, University Hospital and University of Zurich, Switzerland

2 Clinical Department of Neurology, Medical University of Innsbruck, Austria

3 Medica Medizinische Laboratorien Dr. F. Kaeppeli AG, Zurich, Switzerland

Background and aim: Myelin oligodendrocyte glycoprotein antibody-associated autoimmune disease (MOGAD) is a monophasic or relapsing inflammatory demyelinating disease of the central nervous system (CNS). MOGAD causes several atypical, i.e., non-multiple sclerosis (MS), demyelinating CNS syndromes including (often recurrent) optic neuritis, myelitis, aquaporine 4 (AQP4)-seronegative neuromyelitis optica (NMO)-like disease, (brainstem) encephalitis, acute disseminating encephalomyelitis (ADEM)-like disease, and other syndromes. Today it is considered a disease entity separate from multiple sclerosis or classical AQP4-seropositive NMO spectrum disorder because of distinct immunological, histopathological, serological, clinical and paraclinical features, as well as distinct therapy responses and prognosis. Recently, a large multi-center study characterized 163 cerebrospinal fluid (CSF) findings from 100 adult MOGAD patients and found no cases with a positive measles/rubella/zoster (MRZ) reaction and only in $10 \%$ of cases signs of intrathecal IgG production, two features specifically or frequently found in MS. We aimed to reproduce these findings at our center.

Material and methods: We retrospectively assessed demographic, clinical and CSF findings from clinical routine in a cohort of 24 adult patients with MOGAD and 120 patients with MS, who were consecutively punctured at our center. CSF-specific oligoclonal bands indicated intrathecal IgG synthesis. A positive MRZ reaction was defined as polyclonal intrathecal production of antibodies reactive to $\geq 2$ of the 3 antigens, i.e., measles (M), rubella $(\mathrm{R})$, and zoster $(\mathrm{Z})$ virus antigens.

Results: MOGAD patients were significantly older (median age 40.9 years, range 14-74) than MS patients (median age 30.8 years, range 17-69). 33.3\% of MOGAD patients had a monophasic disease course (and $66.7 \%$ were relapsing-remitting), whereas $90.8 \%$ of MS patients were relapsing-remitting and $9.2 \%$ were primary-progressive. MOGAD patients showed a significantly lower frequency of CSF-specific oligoclonal bands (16.7\%) than MS patients $(92.5 \%)$. A positive MRZ reaction was found in $42.5 \%$ of MS patients, but in none of the MOGAD patients $(0 \%)$. 
Conclusions: We corroborate previous findings showing absence of MRZ reaction and very low prevalence of intrathecal IgG production in MOGAD patients. These findings highlight the diagnostic usefulness of a positive MRZ reaction as a rule-out parameter for MOGAD and rule-in parameter for MS.

\title{
P24 HaNDL Syndrome: 5 Cases with Negative CSF Testing for Metagenomic Virus
} Sequencing and Autoimmune GFAP Astrocytopathy

\section{Reichen ${ }^{1}$, M. Huber ${ }^{2}$, I. Jelcic ${ }^{1}$}

1 Department of Neurology, University Hospital and University of Zurich, Switzerland 2 Institute of Medical Virology, University of Zurich, Switzerland

Background and aim: Headache and Neurologic Deficits with cerebrospinal fluid Lymphocytosis (HaNDL) syndrome is a rare, self-limited disorder with unknown etiology. Patients present with transient, often stereotypically re-occurring episodes of severe migraine-like headache with neurological deficits, that include hemiparesis, hemiparesthesia and/or dysphasia, and cerebrospinal fluid (CSF) lymphocytosis, typically with a prodromic viral-like illness days before. Therefore, a viral infection of the central nerve system (CNS) has been hypothesized; alternatively, a delayed humoral or cellular immune response cross-reactive to an unknown CNS antigen, that induces an aseptic leptomeningeal inflammation, has been discussed. We aimed to search for signs of viral CNS infection or autoimmune glial fibrillary acidic protein (GFAP) astrocytopathy as the cause of the HaNDL syndrome.

Material and methods: We retrospectively characterized clinical and CSF data of 5 patients with HaNDL syndrome from our institution and tested CSF samples for viral CNS infection using metagenomic next-generation sequencing and for GFAP astrocytopathy using standardized cell-based assay.

Results: In all patients metagenomic next-generation sequencing of DNA and RNA viral sequences and analysis of anti-GFAP-antibodies were negative.

Conclusions: Our case series did not support a viral CNS infection or GFAP-astrocytopathy as the cause of the HaNDL syndrome. The etiology remains elusive.

\section{P25 Diagnostic and Therapeutic Challenges in Susac Syndrome: Experiences from a Case Series}

\author{
T. Neziraj ${ }^{1}$, I.C. Reichen ${ }^{1}$, M. Heider ${ }^{1}$, D. Gramatzki ${ }^{1}$, A, Pangalu ${ }^{2}$, I. Jelcic ${ }^{1}$, \\ M. Weller ${ }^{1}$, L. Steiner ${ }^{1}$
}

1 Department of Neurology, University Hospital and University of Zurich, Switzerland

2 Department of Neuroradiology, University Hospital and University of Zurich, Switzerland

Background: Susac syndrome (SS) is a rare autoimmune endotheliopathy affecting precapillary arterioles in the brain, retina and inner ear defined by a characteristic clinical triad of encephalopathy, retinopathy and sensorineural hearing loss. The aim of the study was to evaluate the frequency of characteristic clinical and diagnostic findings, the use of early immunosuppressive therapy and the final outcome in patients with SS treated at our center.

Methods: Six patients who were diagnosed with SS between May 2014 and April 2021 were included in this retrospective single-center study.

Results: The initial clinical presentation of all patients was unspecific (e.g., migraine-like headache, behavioral alterations, focal neurological signs) and included only 1-2 of the 3 clinical hallmark features, i.e., encephalopathy, retinopathy and/or sensorineural hearing loss. However, subclinical SS-typical pathological changes were found upon fluorescein angiography (FA) of the eyes and audiogram in all 6 patients. At the onset of the disease, 
cranial MRI (cMRI) findings were unspecific in 4 of 6 patients. During disease progression, 2 of 4 patients with initial unspecific cMRI findings developed all SS-typical cMRI signs, i.e., (1) "snowball-like" white matter lesions (with involvement of the corpus callosum), (2) deep grey matter lesions and (3) leptomeningeal enhancement within 7 to 25 days. Four of 6 patients were treated with glucocorticoids and intravenous immunoglobulins (IVIG). Three of those 4 patients needed additional higher-intensity immunosuppressive therapy for clinical improvement and stabilization, i.e., cyclophosphamide, rituximab or mycophenolate mofetil. Two of 6 patients were treated with glucocorticoids and additional cyclophosphamide. Residual symptoms persisted in 5 of 6 patients. Two of 6 patients experienced a relapse of disease within a range of 3-18 months after disease onset.

Conclusion: The rare incidence of SS and the variable clinical and MRI findings at disease onset may delay an immunomodulatory therapy. FA or audiogram should be evaluated early in the diagnostic process since subclinical pathological changes are common at disease onset. Moreover, cMRIs should be repeated early as typical cMRI findings can develop within days. In clinical practice, early intensive immunosuppressive therapy should be considered in addition to glucocorticoids and IVIG in order to achieve clinical remission and stabilization.

\title{
P26 Premotor and Fronto-Striatal Mechanisms Associated with Presence Hallucinations in Dementia with Lewy Bodies
}

\author{
N. Nicastro ${ }^{1}$, G. Stripeikyte ${ }^{2}$, F. Assal ${ }^{3}$, V. Garibotto ${ }^{3}$, O. Blanke ${ }^{2}$ \\ 1 Hôpitaux Universitaires de Genève, Switzerland \\ 2 Ecole Polytechnique Federale de Lausanne, Switzerland \\ 3 Hôpitaux universitaire de Genève, Switzerland
}

Introduction: Presence hallucinations (PH) are frequent in dementia with Lewy bodies (DLB), implying the vivid sensation that somebody is nearby when no one is actually present and can neither be seen nor heard. $\mathrm{PH}^{\prime}$ 's pathomechanisms are unclear, but recent studies have defined key fronto-temporal areas contributing to the occurrence of $\mathrm{PH}$ (PH-network) and tested their relevance in patients with Parkinson's disease (PD), notably. Methods: For the present study, we included 34 DLB subjects (10 with $\mathrm{PH}(\mathrm{PH}+)$; 24 without PH (PH-)), who underwent 18F-FDG PET and 123I-FP-CIT SPECT imaging. We performed 18F-FDG PET group comparisons, as well as interregional correlation analyses using 18F-FDG PH-network regions as a seed.

Results: PH+ versus PH- had reduced 18F-FDG uptake in precentral, superior frontal and parietal gyri, involving ventral premotor cortex (vPMC) of the PH-network that showed strongly reduced functional connectivity with bilateral cortical regions. 18F-FDG vPMC uptake was negatively correlated with caudate 123I-FP-CIT uptake in PH+ $(p=0.028)$ and interregional correlation analysis seeding from the vPMC showed widespread frontoparietal 18F-FDG decreases in PH+.

Conclusions: the present study advances the neuroscientific understanding of $\mathrm{PH}$ in DLB, by revealing fronto-parietal metabolic changes and especially alterations of the vPMC, a key hub in an independently defined PH-network that has also been shown to be altered in PD patients. In addition, our work revealed that reduced vPMC 18F-FDG uptake was associated with decreased glucose metabolism in widespread cortical regions. Another important finding revealed the involvement of striatal function, showing that $\mathrm{PH}$ are additionally associated with alterations in fronto-striatal connectivity. Sensorimotor processes involving premotor cortex and fronto-striatal connectivity are at play for $\mathrm{PH}$ in DLB. While visual hallucinations are rather related to disruption of posterior brain regions, the present study suggests that $\mathrm{PH}$ pathophysiology is compatible with neurocognitive hallucination models that associate a disruption of frontal communication with a specific PH-network. Future studies should investigate the sensitivity of different patients with 
dementia to robot-induced $\mathrm{PH}$ and acquire functional and structural MRI to corroborate the potential of symptomatic and robot-induced $\mathrm{PH}$ in the understanding and diagnosis of psychosis across neurodegenerative diseases.

\title{
P27 High Efficacy Therapies for Multiple Sclerosis in Switzerland-Perception \& Expectations from Swiss Neurologists
}

\section{G. Perriard, M. Felder, N. Schäfer, M. Arzt}

Novartis Pharma Schweiz AG, Basel, Switzerland

Background: In 2021, 17 disease-modifying therapies (DMT) are available in Switzerland to treat patients suffering from multiple sclerosis. In the light of recent new launches of highly effective therapies (HET), this survey aims at better understanding the perception of the benefit-risk profile of the different MS drugs and the treatment preferences of Swiss neurologists. More specifically, we wanted to understand the perceived limitations and strengths of anti-CD20 therapies (aCD20).

Methods: We conducted a structured interview study containing 9 questions related to DMT efficacy, safety, tolerability, and ease of use, with a focus on aCD20. Randomly picked neurologists practicing in all parts of Switzerland in an office or hospital setting were interviewed. The questionnaires were collected between July 2020 and April 2021.

Results: 35 neurologists were interviewed, with 63\% from Deutschschweiz, 29\%, from Romandie, and $9 \%$ from Ticino. $71 \%$ were practicing in an office-based context, $26 \%$ in a hospital. According to participants, the average efficacy ranking by DMT class is (high to low): 1. aCD20, 2. Other antibody therapies, 3. Orals and 4. Injectables. Regarding safety, injectables were rated highest, followed by aCD20, orals and other antibody therapies.

Focusing on aCD20, $47.4 \%$ of RR-MS patients were reported to be treated first line, $73 \%$ second line and $71.7 \%$ third line or later. The main reported driver for first line use was high disease activity (28/35 neurologists).

Patient preferences, according to neurologists, regarding the administration of anti-CD20 therapies are: $84.4 \%$ s.c. injection vs. $15.6 \%$ i.v. infusion; $96 \%$ self-administration vs. $4 \%$ infusion-center/hospital; $59.4 \%$ monthly vs. $40.6 \%$ every 6 months.

Perceived unmet needs of aCD20 are: lack of long-term data (33.3\%), other [incl. organizational burden, follow up, improvement of symptoms, price] (21\%), onboarding procedure $(18.1 \%)$, safety $(12.1 \%)$, route of administration $(9 \%)$, compliance $(3 \%)$ and monitoring $(3 \%)$. Conclusions: Although aCD20 are considered as having the best efficacy-safety profile compared with other DMT classes, they are not used as first line treatment for the majority of patients and are still restricted to highly active patients. Next-generation anti-CD20 therapy could therefore address some of the limitations expressed by the interviewed neurologists and, hence, may increase acceptance of earlier use of HET for treating patients suffering from multiple sclerosis.

P28 Advanced Neuroimaging, Clinical and CSF Biomarkers to Predict Symptom Reversibility in Idiopathic Normal Pressure Hydrocephalus

\author{
A. Griffa ${ }^{1}$, G. Bommarito ${ }^{1}$, F. Assal ${ }^{2}$, F.R. Herrmann ${ }^{1}$, D. Van De Ville ${ }^{3}$, G. Allali ${ }^{2}$ \\ 1 University of Geneva (UNIGE), Switzerland \\ 2 HUG Hôpitaux Universitaires Genève, Switzerland \\ 3 Ecole Polytechnique Fédérale de Lausanne (EPFL), Switzerland
}

Context \& Objectives: Idiopathic Normal Pressure Hydrocephalus (iNPH), the leading cause of reversible dementia in aging, is characterized by gait, cognitive and urinary impairments. Symptoms can be reverted by CSF removal. However, diagnosis and patient selection for shunting are challenging: iNPH symptoms are unspecific and found in other 
disorders, such as Alzheimer's disease (AD), which frequently occurs as comorbidity. Identifying reliable predictors of symptom reversal is of primary importance to improve the diagnosis and clinical management of iNPH. The objectives of this study are to identify clinical and neuroimaging parameters predicting symptom improvement after a CSF tap test (CSFTT) and assess the role of AD pathology on symptom improvement in iNPH.

Materials and Methods: A total of 30 consecutive patients ( $79.7 \pm 6.3$ years, 12 women) with a diagnosis of possible or probable iNPH were recruited at Geneva University Hospitals and underwent, on the same day, a comprehensive neuropsychological assessment; a quantitative gait assessment; an MRI session including T1w, T2w, DWI, ASL, restingstate fMRI; a CSFTT with removal of $40 \mathrm{~mL}$ of CSF. Ventricular and sulcal morphometry, periventricular white matter (WM) microstructure (fiber orientation; lesions load), and default mode network function (perfusion; functional connectivity) were quantified from MRI data with state-of-the-art methods. AD biomarkers including amyloid-beta-42, total and phosphorylated tau were assessed in the CSF. Walking speed and timed up and go (TUG) test were performed before and the day after the CSFTT. Patients were labeled as responders if they improved at least $10 \%$ in walking speed or TUG after CSFTT.

Results: 16 patients (53\%) responded positively to CSFTT. A linear support vector machine classifier including clinical and neuroimaging features achieved $75 \%$ sensitivity and $64 \%$ specificity in predicting gait improvement after CSFTT. Gait, WM lesion load and periventricular fiber organization contributed the most to prediction, while cognition and brain function contributed the least. There was no difference in $\mathrm{AD}$ biomarkers between patients who improved or not after CSFTT.

Conclusions: Combined gait and WM parameters achieve robust, although moderate prediction accuracy for symptom improvement after CSFTT. We found no association between cognition, Alzheimer's pathology and iNPH reversibility. This study encourages future investigation on the prediction of shunt surgery outcome.

P29 The Behavioural Dysfunction Questionnaire Highly Discriminates Early-Stage Behavioural Variant Frontotemporal Dementia from Alzheimer's Disease Dementia and Major Depressive Disorder

A. Semenkova ${ }^{1}$, O. Piguet ${ }^{2}$, A. Johnen ${ }^{3}$, S. Bruchmann ${ }^{3}$, M.L. Schroeter ${ }^{4}$, C. Linnemann ${ }^{5}$, M. Mühlhauser ${ }^{5}$, T. Sauer ${ }^{5}$, M. Baumgartner ${ }^{6}$, S. Anderl-Straub ${ }^{7}$, M. Otto ${ }^{8}$, S. Zimmerer ${ }^{9}$, A. Felbecker ${ }^{9}$, R.W. Kressig ${ }^{10}$, M. Berres ${ }^{11}$, M. Sollberger ${ }^{12}$

1 Memory Clinic, University Department of Geriatric Medicine FELIX PLATTER/Faculty of Psychology, University of Basel, Switzerland

2 School of Psychology and Brain and Mind Centre, The University of Sydney, Australia

3 Clinic for Neurology, Muenster University Hospital, Germany

4 Clinic for Cognitive Neurology, University Hospital Leipzig/Max Planck Institute for

Human Cognitive and Brain Sciences, Germany

5 University Psychiatric Clinic, Basel, Switzerland

6 memoryclinic-sonnweid, Wetzikon, Switzerland

7 Department of Neurology, University of Ulm, Germany

8 Department of Neurology, University of Ulm/Department of Neurology, University Hospital Halle, Germany

9 Clinic of Neurology and Neurophysiology, Canton Hospital St. Gallen, Switzerland

10 University Department of Geriatric Medicine FELIX PLATTER, Basel, Switzerland

11 Faculty of Mathematics and Technology, University of Applied Sciences Koblenz, Germany

12 Memory Clinic, University Department of Geriatric Medicine FELIX PLATTER/ Department of Neurology, University Hospital Basel, Switzerland

Background and Objectives: Early-stage behavioural variant frontotemporal dementia (bvFTD) is quite often misdiagnosed. Thus, there is a need for new diagnostic instruments. 
Based on the revised diagnostic criteria for bvFTD, we developed the Behavioural Dysfunction Questionnaire (BDQ). In this explorative study, we aimed to: (a) determine the best scoring and analytical method for the BDQ to discriminate between patients with and without bvFTD, and (b) compare its discriminative strength with that of the Frontal Behavioral Inventory (FBI), the most established bvFTD questionnaire.

Materials and Methods: 38 patients with bvFTD, 54 with Alzheimer's disease (AD) dementia and 40 with major depressive disorder (MDD) were recruited. We used nine different analytical methods: First, we calculated BDQ items with or without inclusion of a time component: (a) without time criterion, (b) with time criterion by correcting for personality characteristics, and (c) with time criterion by correcting for symptom appearance later than three years after clinical onset. Next, we generated two variables [i.e., the BDQ global score (numerical variable including behavioural disorders) and the Behavioural Domain score (categorical variable including behavioural domains)]. Each variable was generated separately with the three differently calculated items. With these six variables, we performed ROC analyses. Finally, we combined the BDQ global score and Behavioural Domain score for each time criterion in a logistic regression model, and performed ROC analyses on the three scores.

Results: Models without time component and models including the personality time component discriminated better than the model including the three years' time component. BDQ global and BDQ global \& Behavioural Domain score models discriminated better than the Behavioural Domain score model. These two models, either without time component $(\mathrm{AUC}=0.87)$ or with the personality time component $(\mathrm{AUC}=0.86)$, discriminated similarly to the model with the FBI variable $(\mathrm{AUC}=0.81)(p=0.11)$.

Conclusions: Adding the time point of symptom presentation to the scoring, as suggested by the revised diagnostic criteria for bvFTD, did not improve the discriminative strength of the BDQ. The BDQ global score without time component highly discriminated bvFTD from AD dementia and MDD. Next, we aim to improve the discriminative strength of the $\mathrm{BDQ}$ by taking a data-driven approach in a larger dataset with more patient groups.

P30 The Personalized Assessment of Regional Cerebral Perfusion and Outlier Symmetry in Retired Canadian Football League Players

\section{E. Danielli ${ }^{1}$, B. Padrela ${ }^{2}$, M. Doughty ${ }^{1}$, J. Petr ${ }^{3}$, H.J. Mutsaerts ${ }^{2}$, S. Ulmer ${ }^{4}$, MD Noseworthy ${ }^{1}$}

1 McMaster University, Hamilton, Canada

2 Amsterdam University Medical Center, Amsterdam, Netherlands

3 Helmholtz-Zentrum Dresden-Rossendorf, Germany

4 neurorad.ch, Switzerland

Background \& Goals: High contact sports can result in athletes sustaining concussions and countless sub-concussive blows, leading to cumulative neurological damage and dementia [1]. This study aimed to use cerebral blood flow (CBF) measured with ASL and a Z-scoring approach to quantify focal brain damage and left/right symmetry in retired Canadian Football League (rCFL) players. It was hypothesized that CBF would be symmetric and have regional hypoperfusion due to the subjects' history of repetitive head trauma.

Materials \& Methods: Seventeen rCFL players ( $100 \%$ male, aged $58 \pm 6.15$ y) were scanned using a 3T GE Discovery MR750 MRI and a 32-channel head coil. 3D T1-weighted fSPGR and 3D pseudo-continuous ASL (pCASL) scans acquired. The pCASL scans were processed using ExploreASL to produce spatial covariance (CoV) CBF quantification [2,3]. Twelve concussion-related regions-of-interest (ROIs) were selected from the Harvard-Oxford (HO) and Hammers (HS) Atlases [4]. ROI CBF was analyzed for the left and right hemispheres separately and bilaterally. Prior to Z-scoring, ROIs that failed Shapiro-Wilk normality testing or had insufficient data were excluded. The ROI CoV values were normalized 
to each subject's grey matter CoV CBF. A Z-scoring approach was applied in MATLAB with the bilateral $\mathrm{CoV}$ value as the comparable metric. Z-score outliers that fell 2, 3 or 4 standard deviations from the bilateral means were classified as mild (1), moderate (2), or severe (3) injury burden (IB). A subject-specific IB symmetry index was calculated as: $(\mathrm{L}-\mathrm{R}) /(0.5(\mathrm{~L}+\mathrm{R}))$. Normality and correlation tests were performed with RStudio, where age, type of positional head impact, and career length were used as covariates.

Results: The inferior frontal gyrus pars opercularis (IB =9), cingulate gyrus (anterior) (IB $=5$ ) and inferior temporal gyrus posterior division $(\mathrm{IB}=4)$ had the greatest IBs. IB was symmetrical $(0.13 \pm 1.33)$, with more positive outlier Z-scores (positive $=19$; negative $=14)$. Left IB and negative Z-scores were significantly correlated $(r=+0.604)$, and right IB and positive Z-scores were significantly correlated $(\mathrm{r}=+0.706)$.

Conclusions: Group IB was symmetrical, but subject-wise IB was often unilateral. In agreement with literature, the cingulate cortex and inferior frontal and temporal gyri may be especially vulnerable to injury [4]. Further studies may analyze concussion CBF in relation with DTI and fMRI.

\title{
P31 Neurological Signal Complexity as a Diagnostic Tool of Alzheimer's Disease
}

\author{
E. Samson ${ }^{1}$, N. Simard ${ }^{1}$, K. Zukotynski ${ }^{1}$, S. Krumm ${ }^{2}$, S. Ulmer ${ }^{3}$, M. Noseworthy ${ }^{1}$ \\ 1 McMaster University, Hamilton, Canada \\ 2 University of Basel, Switzerland \\ 3 University hospital of Schleswig-Holstein, Germany
}

\begin{abstract}
Alzheimer's Disease (AD) is a progressively debilitating neurodegenerative disorder that impairs an individual's cognitive function. While diagnosis is typically based on clinical findings, gaps in our understanding of the pathophysiological changes underlying disease onset remain. A tool for early diagnosis could improve this understanding in addition to providing better patient outcome. One possibility is through the use of temporal complexity analysis of resting state fMRI (rsfMRI), where reduced complexity is indicative of reduced brain functional capacity and increased disease burden.

rsfMRI signals can be classified as statistical fractals since they express degrees of selfsimilarity/self-invariance. Regional temporal complexity can be determined through the signal Hurst exponent $(0<H \leq 1)$ which is based on the temporal fractal dimension (FD). It has previously been shown that suspected AD patients have reduced time domain complexity in the amygdala. Our current work focuses on investigating whole brain regional analysis of complexity. We hypothesize that AD impacts specific brain regions creating a unique signature, evident in $\mathrm{H}$ frequency domain variants. Thus, regional reduced brain complexity could be a metric for early diagnosis. Suspected AD patients $(n=24)$ with moderate or early-stage AD (9 male; mean age 76, MMSE = $22+3$ ) were scanned using a 3T MRI and 8-channel head coil. High-resolution 3D IR-prepped T1-weighted and restingstate BOLD data were acquired. TBIFinderTM software was used to determine regional temporal complexity and calculation of overall disease burden (ODB). The ODB was the sum of regions having a $\mathrm{H}$ z-score $<-1.68$ standard deviations from sex/age matched healthy controls. Personalized regional brain $\mathrm{H}$ anomalies were also compared against regional brain hippocampal and amygdala volumes determined using NeuroQuantTM. Affected regions having reduced $\mathrm{H}$ included the secondary somatosensory cortex (79\% of AD patients), Broca's areas (75\%), amygdala (58\%), insula (50\%) and hippocampus $(45 \%)$. Significant metrics in predicting ODB were hippocampal volume $(p<0.002)$ and age $(p<0.0005)$, with interaction terms of age:sex(female) $(p<0.02)$ and hippocampal volume:sex(female) $(p<0.002)$ showing that sex is important only when considered with other variables. In conclusion, rsfMRI complexity is a useful metric in assessing moderate or early-stage AD. Future analysis needs to include brain volumetric measures from more regions.
\end{abstract}


P32 Cerebellar Structural and Functional Damage Present in Retired Canadian Football League (CFL) Players with a History of Concussion

\author{
N. Simard ${ }^{1}$, E. Danielli ${ }^{2}$, M. Doughty ${ }^{2}$, S. Ulmer ${ }^{3}$, M.D. Noseworthy ${ }^{4}$ \\ 1 TBIFinder inc., Research and Development, Hamilton, Canada \\ 2 McMaster University, Hamilton, Canada \\ neurorad.ch, Neuroradiology, Zurich, Switzerland \\ 4 St. Joseph's Healthcare Hamilton, McMaster University, Canada
}

IntroSport-related concussion can cause unique and potentially lasting deficits that can lead to dementia and other neurodegenerative conditions [1,2]. The purpose of this study was to identify and quantify structural and functional abnormalities present in the cerebellum in retired professional athletes. It was hypothesized that structural and functional deficits in the cerebellum would match clinical presentation.

Seventeen retired Canadian Football League (rCFL) players (all male, aged $58.3 \pm 6.2$ ) were scanned using a 3T GE MR750 MRI and 32-channel head coil. High-resolution 3D T1-weighted, 60-direction diffusion tensor imaging (DTI), and resting state functional MRI (rsfMRI) scans were acquired for each subject. Focusing on cerebellum, images were processed using TBIFinder software which provides a severity score, scaled from $0-6$, and location of cerebellar abnormalities based on DTI (structure) and rsfMRI (function) scans $[3,4]$. The MNI152 Cerebellar atlas was used to segment ROIs to investigate each lobe, along with the vermis, and right and left sides of the cerebellum [5]. IMPACT, SCAT3, and demographic data were also collected for each subject in order to correlate TBIFinder results with clinical scores. Further, using RStudio a correlation analysis was performed for each TBIFinder metric with age, sport position, and career length as covariates.

From the DTI data, all subjects $(n=17)$ were identified as having an abnormality present in their cerebellum. Subjects with any cerebellar region having a severity score above $3(n=5$, $p<0.0027)$ observationally performed worse on their IMPACT and SCAT3 tests. Similar results were found for the rsfMRI data where 6 subjects presented with severity scores above $3(p<0.0027)$. The ROI with the highest frequency of abnormality in this population (14 of 17 players) was the vermis of the anterior lobe $(p<0.0455)$. No correlations with cerebellar hemisphere, age, position, or career length were noted.

Although there are age-related decreases in normal aging, [6] our study identified decreases in structural and functional integrity in former professional athletes surpassing that found in normal aging brains. Further research must be conducted to explore the relationship between more in-depth neuropsychological testing, TBIFinder abnormality detection, and regional results.

P33 COVID-19 Infections and Vaccinations in the Swiss Multiple Sclerosis Cohort Study

V. Epple ${ }^{1}$, L. Hauss ${ }^{1}$, A. Orleth ${ }^{1}$, P. Benkert ${ }^{2}$, D. Jorganxhi ${ }^{3}{ }$, N. Sanderson ${ }^{4},{ }^{\prime}$ K. Ivánek ${ }^{4}$, L. Kappos ${ }^{3}$, L. Achtnichts ${ }^{5}$, J. Saxer ${ }^{5}$, C. Pot ${ }^{6}$, P. Lim Dubois-Ferrière ${ }^{6}$, P.H. Lalive ${ }^{7}$, G. Breville ${ }^{7}$, C. Bridel ${ }^{7}$, C. Gobbi ${ }^{8}$, C. Zecca ${ }^{8}$, S. Mueller ${ }^{9}$, F. Gruenenfelder ${ }^{9}$, T. Derfuss ${ }^{1}$, C.T. Berger ${ }^{4}$, R. Pedotti ${ }^{10}$, C. Raposo ${ }^{10}$, K. Leuzinger ${ }^{4}$, H.H. Hirsch ${ }^{4}$, J. Kuhle ${ }^{1}$, M. Mehling ${ }^{1}$

1 Neurologic Clinic, University Hospital Basel, Switzerland

2 Clinical Trial Unit, Department of Clinical Research, University Hospital Basel, Switzerland

3 Research Center for Clinical Neuroimmunology and Neuroscience Basel, University Hospital Basel and University Basel, Switzerland

4 Department of Biomedicine, University Hospital and University of Basel, Switzerland

5 Department of Neurology, Cantonal Hospital Aarau, Switzerland 
6 Service of Neurology, Department of Clinical Neurosciences, Lausanne University Hospital (CHUV) and University of Lausanne, Switzerland

7 Department of Neurology, University Hospital of Geneva (HUG), Switzerland

8 Department of Neurology, Neurocenter of Southern Switzerland, Lugano, Switzerland

9 Department of Neurology, Cantonal Hospital St. Gallen, Switzerland

10 F. Hoffmann-La Roche Ltd., Switzerland

Background: COVID-19 disease course in MS has been described in various cohorts. Limited data is available on humoral immune responses following SARS-CoV-2 infection and vaccination.

Objectives: To determine the rate of confirmed SARS-CoV-2 infection and severity of COVID-19 in a cohort of MS patients and to quantify SARS-CoV-2-specific antibody response. Methods: The study is nested within the Swiss MS Cohort, a nationwide multicenter study that has recruited 1504 persons with MS (pwMS) since 2012. PCR-confirmed SARSCoV-2 infections, severity of COVID-19 according to the WHO clinical progression scale and immunizations with SARS-CoV-2 vaccines were captured by questionnaires used for interviews every 6 or 12 months. Anti-SARS-CoV-2 spike protein and nucleocapsid antibody levels will be determined by electrochemiluminescence immunoassay (ECLIA) (Elecsys, Anti-SARS-CoV-2, Roche Diagnostics) in sera of all participants.

Results: Between February 2021 and April 2021, study questionnaires were completed for 253 pwMS (median age 47 years, 162 female). 211 pwMS (83\%) had a relapsing, 25 $(10 \%)$ a secondary progressive, $13(5 \%)$ a primary progressive disease course and $4(2 \%)$ a clinically isolated syndrome. Median disease duration was 12 years and median EDSS was 2.5. $218(86 \%)$ pwMS were treated with DMTs: Ocrelizumab (27\%), fingolimod (26\%), dimethyl fumarate (15\%), rituximab (10\%), natalizumab (9\%), other DMTs $(13 \%) .15(5.9 \%)$ of 253 pwMS had a positive SARS-CoV-2 PCR test since March 2020. In these pwMS, COVID-19 severity ranged from 1-10 on the WHO clinical progression scale: 1 pwMS was asymptomatic, 10 pwMS were symptomatic as outpatients ( 8 independently, 2 needed assistance), 3 pwMS were hospitalized (1 without oxygen therapy, 2 with oxygen by mask or nasal prongs) and 1 pwMS died. By April 2021, 24 and 38 pwMS received one and two doses of SARS-CoV-2 mRNA vaccines, respectively.

Conclusions and outlook: Since start of the pandemic, rate of PCR-confirmed SARS-CoV-2 infection in our sample was slightly lower compared to incidence of laboratory-confirmed cases in Switzerland. The majority of pwMS had mild COVID-19. The study will continue until 2024 and by ECTRIMS 2021, we anticipate a doubling of completed questionnaires and to report preliminary results of serological measurements. This will allow us to present vaccine- and natural infection induced serological anti-SARS-CoV-2 responses in pwMS and assess differences related to various DMTs or COVID-19 severity.

\section{P34 The Biological Substrate of the Motoric Cognitive Risk Syndrome: An} Amyloid-/Tau-PET and MR Imaging Study

G. Bommarito, V. Garibotto, G.B. Frisoni, F. Ribaldi, S. Stampacchia, F. Assal, S. Armand, G. Allali, A. Griffa

Geneva University Hospitals and University of Geneva, Switzerland

Background and Objectives: The motoric cognitive risk (MCR) syndrome-characterized by slow gait and cognitive complaint-represents a pre-dementia condition, able to identify older adults at risk of developing dementia (1). Previous studies variably related MCR to an increased risk of Alzheimer's disease (AD), vascular dementia or both. However, the biological substrate of MCR is still matter of debate and the relationship between AD biomarkers and MCR has not been investigated yet. In this study we explored the association between $\mathrm{MCR}$ and imaging features of $\mathrm{AD}$, vascular disease or unspecific neurodegeneration. 
Methods: Twenty participants with cognitive complaints referring to the Memory Center of the Geneva University Hospitals were recruited. Patients were classified as MCR+ or MCR - based on their gait speed, with respect to reference values derived from a local cohort of healthy older adults, assessed using the same gait protocol. Amyloid and tau uptakes were derived from PET imaging. White matter hyperintensities $(\mathrm{WMH})$, temporal atrophy, lateral ventricular volume (LVV) (2) and microstructural damage of gait-related white matter tracts (i.e., the corpus callosum, cortico-spinal tract and superior corona radiata) $(3,4)$ were derived from MRI. Imaging parameters were compared between the two groups.

Results: MCR $+(n=8$, mean age $72.5 \pm 6.3$ years, 2 females $)$ and MCR $-(n=12$, mean age $73.7 \pm 6.7$ years, 5 females) patients did not differ in terms of amyloid or tau burden, temporal atrophy, WMH volume or microstructural damage at the level of the corpus callosum and cortico-spinal tract. MCR+ patients had significantly larger LVV and higher mean diffusivity values at the level of the superior corona radiata $(p=0.041$ and $p=0.018$, respectively), compared to $\mathrm{MCR}-$.

Conclusions: MCR could be not related uniquely to AD or vascular pathologies. Ventricular enlargement and the damage of the adjacent white matter tracts, more than a dementia sub-type specific pathogenic pathway, could characterize MCR. These imaging features should be included in future larger studies to confirm these findings.

\title{
P35 Post-Approval Safety of Cladribine Tablets with Particular Reference to COVID-19 Outcomes: An Update
}

\author{
G. Giovannoni ${ }^{1}$, J. Berger ${ }^{2}$, T. Leist ${ }^{3}$, D. Jack ${ }^{4}$, A. Galazka ${ }^{4}$, A. Nolting ${ }^{4}$, D. Damian ${ }^{5}$ \\ 1 Blizard Institute, Barts and The London School of Medicine and Dentistry, Queen Mary \\ University of London, London, UK \\ 2 Department of Neurology, Perelman School of Medicine, University of Pennsylvania, \\ Philadelphia, PA, USA \\ 3 Division of Clinical Neuroimmunology, Jefferson University, Comprehensive MS Center, \\ Philadelphia, PA, USA \\ 4 Merck KGaA, Darmstadt, Germany \\ 5 EMD Serono Research \& Development Institute, Inc., Billerica, MA, USA, an affiliate of \\ Merck KGaA, Darmstadt, Germany
}

Introduction: The safety profile of cladribine tablets $10 \mathrm{mg}$ (CladT; $3.5 \mathrm{mg} / \mathrm{kg}$ cumulative dose over 2 years) from the phase III clinical development programme for relapsing multiple sclerosis (MS) is well characterised (Cook et al. MSaRD 2019; 29: 157-167). Additional real-life safety data have accrued since the approval of CladT in $>80$ countries worldwide. Objectives: To update on the post-approval safety profile of CladT in patients with relapsing MS, with particular reference to COVID-19.

Methods: We previously reported on outcomes for 46 CladT-treated patients with relapsing MS and confirmed or suspected COVID-19 (Jack et al. MSaRD 2020; 46: 102469). Here, we update on these findings, to 15 March 2021, based on cases reported to the Merck KGaA Global Patient Safety Database. Cases meeting the criteria of hospitalized, medically significant, or fatal were designated as serious, and outcomes were classified as per usual pharmacovigilance practice. Serious and non-serious adverse events (AEs) from postapproval sources (including spontaneous individual case safety reports, non-interventional post-marketing studies, and reports from other solicited sources) are also presented.

Results: As of 15 March 2021, the safety database included 367 reported cases of COVID-19 in CladT-treated patients (confirmed by test, $n=219$; serious cases, $n=49$ ); 6 patients had symptoms compatible with COVID-19 but were not evaluated further since they were subsequently reported to have negative PCR tests. Of 361 evaluable patients, a total of 187 
(52\%) were recovered/recovering at the time of reporting; there was 1 fatality in a patient with suspected COVID-19.

To date, 3357 AEs have been reported for the first 18,463 patients who received CladT post-approval; 435 (13\%) of these events were serious. Crude incidences for AEs of special interest: severe lymphopenia, $0.21 \%$; herpes zoster, $1.07 \%$; tuberculosis, $0.05 \%$; severe infections, $1.23 \%$; progressive multifocal leukoencephalopathy, $0 \%$; opportunistic infections, $0.04 \%$; malignancies, $0.23 \%$; and congenital anomalies, $0 \%$.

Conclusions: Regarding COVID-19, CladT-treated patients do not appear to be at greater risk of serious disease and/or a severe outcome vs. the general and MS populations. Overall, the post-approval safety profile of CladT is consistent with previously published safety findings from the clinical development programme and ongoing phase IV studies.

\section{P36 Evobrutinib Significantly Reduces Relapses and Magnetic Resonance Imaging Outcomes in Patients with Multiple Sclerosis: Association with Baseline Neurofilament Light Chain Levels}

\section{J. Kuhle ${ }^{1}$, L. Kappos ${ }^{2}$, X. Montalban ${ }^{3}$, Y. Li ${ }^{4}$, K. Thangavelu ${ }^{4}$, Y. Hyvert ${ }^{5}$, D. Tomic ${ }^{5}$}

1 Neurologic Clinic and Policlinic, MS Center and Research Center for Clinical Neuroimmunology and Neuroscience Basel (RC2NB), University Hospital Basel, and University of Basel, Basel, Switzerland

2 Research Center for Clinical Neuroimmunology and Neuroscience Basel (RC2NB), Departments of Medicine, Clinical Research and Biomedical Engineering, University Hospital Basel, and University of Basel, Switzerland

3 Department of Neurology-Neuroimmunology, Centre d'Esclerosi Múltiple de Catalunya (Cemcat), Hospital Universitario Vall d'Hebron, Barcelona, Spain

4 EMD Serono Research \& Development Institute, Inc., Billerica, MA, USA (an affiliate of Merck KGaA, Darmstadt, Germany)

5 Merck KGaA, Darmstadt, Germany

Introduction: Evobrutinib (EVO) is a highly selective Bruton's tyrosine kinase (BTK) inhibitor. In a post-hoc analysis of a phase II randomized trial (NCT02975349), EVO $75 \mathrm{mg}$ twice-daily (BID) significantly lowered blood neurofilament light chain (NfL) levels at weeks 12 and 24. NfL is a biomarker of neuro-axonal damage in multiple sclerosis (MS). Objective: To evaluate the prognostic value of baseline blood NfL levels on clinical relapse and magnetic resonance imaging (MRI) lesion activities, and to further evaluate the treatment effect of evobrutinib in MS.

Methods: The analysis included patients in the modified intent-to-treat population with $\mathrm{NfL}$ values at baseline (excluding the dimethyl fumarate arm). Patients were grouped by high dose (EVO $75 \mathrm{mg}$ once-daily (QD) or $75 \mathrm{mg}$ BID) or placebo/low dose (placebo or EVO $25 \mathrm{mg}$ QD). Baseline NfL was measured blinded to treatment (Simoa NF-light ${ }^{\mathrm{TM}}$ ). Patients were stratified by geometric mean baseline NfL levels as high NfL $(\geq 11.36 \mathrm{pg} / \mathrm{mL})$ or low $\mathrm{NfL}(<11.36 \mathrm{pg} / \mathrm{mL})$, to evaluate the effect on qualified relapses over 24 weeks, and on gadolinium-enhancing (Gd+) T1 lesions and T2 lesions over weeks 12, 16, 20 and 24. Treatment effect was also evaluated stratified by the baseline NfL groups.

Results: The NfL analysis population included 162 patients. Patients with high NfL had higher disease burden at baseline, and higher levels of clinical relapses and MRI lesion activity over 24 weeks. The odds of qualified relapse were significantly reduced for the high dose group versus placebo/low dose, when stratified by baseline NfL levels (odds ratio: 0.12; $p=0.0028$ ). A significant reduction in the cumulative number of $\mathrm{Gd}+\mathrm{T} 1$ lesions and new or enlarging T2 activity, was observed for the high dose group versus placebo/low dose, regardless of baseline NfL: Gd+T1 relative reduction $(\mathrm{RR})=69.4 \%(p=0.0102)$ and $\mathrm{RR}=69.2 \%(p=0.0018)$, new or enlarging $\mathrm{T} 2$ lesions $\mathrm{RR}=73.4 \%(p=0.0012)$ and $\mathrm{RR}=54 \%$ $(p=0.0458)$, for the NfL low and high groups, respectively. 
Conclusions: This is the first study describing the interaction of blood NfL levels and the effects of a BTK inhibitor in patients with MS. The data indicate that higher doses of evobrutinib reduce MRI activity, regardless of baseline NfL levels, and significantly reduce the proportion of patients with relapses versus placebo/low doses, in the high blood NfL level group. They also further support the value of blood NfL levels as a prognostic marker of future disease activity.

P37 Exploratory Analysis of Serum GDF-15 Levels in Patients Receiving Subcutaneous Interferon Beta-1a in the REFLEX Trial

\author{
M.C. Coray ${ }^{1}$, A. Seitzinger ${ }^{2}$, S. Roy ${ }^{2}$, M. Freedman ${ }^{3}$, F. Barkhof ${ }^{4}$, G. Comi ${ }^{5}$, N. De Stefano ${ }^{6}$, \\ L Kappos $^{7}$, J. Kuhle ${ }^{7}$, M. Mehling ${ }^{7}$
}

1 University Hospital Basel and University of Basel, Department of Biomedicine, Basel, Switzerland

2 Merck KGaA, Darmstadt, Germany

3 University of Ottawa, Department of Medicine and the Ottawa Hospital Research Institute, Ottawa, Canada

4 VU University Medical Centre, Department of Radiology, Amsterdam, Netherlands; UCL Institute of Neurology, London, United Kingdom

5 Università Vita-Salute San Raffaele, Ospedale San Raffaele, Department of Neurology and Institute of Experimental Neurology, Milan, Italy

6 University of Siena, Department of Medicine, Surgery and Neuroscience, Siena, Italy

7 University Hospital Basel and University of Basel, Research Center Clinical Neuroimmunology and Neuroscience Basel, and MS Center, Neurologic Clinic and Policlinic, Departments of Medicine, Biomedicine, Clinical Research and Biomedical Engineering, Basel, Switzerland

Introduction: Subcutaneous interferon beta-1a (sc IFN $\beta-1 a$ ) is a well-established diseasemodifying therapy for relapsing multiple sclerosis (MS), with an estimated cumulative exposure of 1,831,698 patient-years (to 30 April 2021). Serum levels of growth differentiation factor 15 (GDF-15), a cytokine with anti-inflammatory effects, are increased in patients with stable MS. However, no information is available concerning GDF-15 in patients treated with sc IFN $\beta-1$ a.

Objectives: To investigate if serum levels of GDF-15 can be used as a predictor of disease stability in patients with clinically isolated syndrome (CIS) treated with sc IFN $\beta$-1a.

Methods: In the REFLEX study (NCT00404352), 480 CIS patients were randomly assigned to treatment with sc IFN $\beta$-1a 44 mcg once weekly (ow, $n=162$ ), 44 mcg three-times weekly (tiw, $n=157)$, or placebo $(n=161)$, for up to 24 months. Time from randomization to clinically definite MS (CDMS), defined by either a second demyelinating event or a sustained increase ( $\geq 1.5$ points) in the EDSS score, was the primary endpoint. In this post hoc exploratory analysis, serum concentrations of GDF-15 were measured by ELISA at baseline and for up to 24 months, including the subgroup of CDMS non-converters vs. converters. All analyses are descriptive.

Results: Whilst median serum GDF-15 levels showed little change between baseline and month 24 in the sc IFN $\beta$-1a 44 mcg ow and placebo cohorts, they increased in the $44 \mathrm{mcg}$ tiw group following treatment start (baseline, $323 \mathrm{pg} / \mathrm{mL}[n=157]$; month $6,391 \mathrm{pg} / \mathrm{mL}[n=135]$; month $24,391 \mathrm{pg} / \mathrm{mL}[n=114])$. This increase was apparent irrespective of baseline GDF-15 levels. Under treatment with sc IFN $\beta-1 \mathrm{a} 44 \mathrm{mcg}$ tiw, CDMS non-converters had persistently higher levels of serum GDF-15 compared with CDMS converters.

Conclusions: In CIS patients, GDF-15 levels increased during treatment with sc IFN $\beta$-1a $44 \mathrm{mcg}$ tiw. The more pronounced increase of GDF-15 levels in CDMS non-converters treated with sc IFN $\beta-1 \mathrm{a} 44 \mathrm{mcg}$ tiw indicates that levels of this anti-inflammatory cytokine 
may serve as a biomarker of treatment response and stable disease under such therapy in early MS.

\section{P38 Effect of Diet on Oral Microbiota in Parkinson's Disease}

\section{Fleury ${ }^{1}$, A. Zekeridou ${ }^{2}$, V. Lazarevic ${ }^{3}$, G. Piumatti ${ }^{1}$, N. Gaïa ${ }^{4}$, E. Berthouzoz ${ }^{4}$, R Goldstein ${ }^{1}$, C. Giannopoulou ${ }^{2}$, A. Mombelli ${ }^{2}$, J. Schrenzel ${ }^{5}$, L. Genton ${ }^{5}$, PR Burkhard ${ }^{5}$}

1 Hôpitaux Universitaire de Genève HUG, Switzerland

2 University Clinic of Dental Medicine, Zurich, Switzerland

3 Geneva University Hospitals, Switzerland

4 University of Geneva (UNIGE), Switzerland

5 Geneva University Hospitals (HUG), Switzerland

Background and Objective: Differences in relative taxa abundance within the oral microbiota have recently been demonstrated in Parkinson's disease (PD) patients compared with healthy controls (HC). The role of diet as a potential confounder remains to be determined. Methods: 16S gene ribosomal RNA sequencing was performed on salivary and subgingival dental plaque samples of 20 PD patients and 20 HC. Dietary intake was assessed by using a self-administered, semiquantitative Food-Frequency Questionnaire. Each participant was examined with thorough neurological, periodontal and dental examinations. For univariate comparisons, the Wilcoxon rank sum test was used. To analyze the relationship between bacterial community profiles and quantitative dietary continuous variables, a distancebased linear model (DISTLM, PRIMER) with 9999 permutation was used. Spearman's rank-order correlations were used to measure the strength and direction of associations between dietary habits and different types of bacteria.

Results: Patients were in the early and mid-stage phases of their disease (Hoehn \& Yahr 2-2.5). Higher saliva disturbances scores were found in patients. Patients and controls did not differ in terms of Body Mass Index, Fat free or Fat Masses, dental or periodontal status. Patients consumed more müesli, green beans/spinach, cauliflower/broccoli, green peas/corn, than controls. These consumptions correlated with chronic constipation measures. Patients ate also more boiled potatoes, cottage cheese, sunflower seed oil and mayonnaise, and drank less beer. More monosaccharides were consumed by patients. No correlations were found between monosaccharide consumption and the level of anxiety, depression, or the dose of dopaminergic treatment. The following food categories correlated with salivary microbiota composition: cauliflower/broccoli, boiled potatoes, sunflower seed oil, monosaccharides and green beans/spinach. No correlation was found with the dental plaque microbiome. Spearman's correlation coefficient demonstrated some positive or negative associations between dietary habits and some bacteria. However, these bacteria were not the ones found in different relative abundances between patients and controls, suggesting that both diet and PD influence differently microbial composition.

Conclusions: Diet influences oral microbiota composition and should be considered systematically in future studies as a potential shaper of oral microbiota, especially salivary microbiome.

P39 Spatial Organisation of Protein Aggregates on Red Blood Cells as Physical Biomarkers of Alzheimer's Disease Pathology

\section{P.N. Nirmalraj ${ }^{1}$, T. Schneider ${ }^{2}$, A. Felbecker ${ }^{2}$}

1 Empa, Swiss Federal Laboratories for Materials Science and Technology, Department Materials meet Life, St Gallen, Switzerland

2 Kantonsspital St. Gallen, Department of Neurology, Switzerland 
Background: Presymptomatic diagnosis is critical for slowing Alzheimer's disease (AD). The key proteins implicated in $\mathrm{AD}$ pathology are amyloid-beta $(\mathrm{A} \beta)$ and tau protein isoforms whose total content can be quantified in cerebrospinal fluid (CSF), blood plasma and serum. Yet, nanoscopic differences in physical properties (size, shape, morphology and prevalence) of the aggregated states of these proteins, which are also important indicators of the disease stage, remains largely unknown in body fluids.

Methods: Here, using a benchtop atomic force microscope (AFM) we profiled protein aggregates adsorbed on red blood cells (RBCs) from 50 patients with cognitive complaints at a memory clinic (age range:53-89, 26 female and 24 male) and compared with 16 healthy individuals (age range: 23-68, 7 female and 9 male).

Results: The AFM images with sub-2nm spatial resolution revealed stark differences in size, shape and distribution of protein aggregates, which was observed to depend on patient age and stage of neurocognitive disorder. Interestingly, crystallographic domains composed of aligned single-fibrils were exclusively detected on RBCs for AD patients aged between $80-89$ years. The prevalence of fibrillar aggregates was negatively correlated with the CSF $A \beta-42 / 40$ ratio and was observed to be significantly higher in the amyloid positive patient category. Using a cut-off of $\geq 40 \%$ prevalence of fibrillar aggregates on RBC the CSF-amyloid status could be classified with $88 \%$ accuracy (sensitivity $100 \%$, specificity $73 \%$ ).

Conclusions: Although our data currently lacks chemical information, the morphological insights gained on the spatial organisation of peptide aggregates on RBCs could still represent a novel screening biomarker of AD pathophysiology.

\title{
P40 Different Awareness Domains Contribute to Explain Emotional and Behavioral Disorders in Mild Alzheimer's Disease
}

\author{
L. Sacco ${ }^{1}$, C.A. Defanti ${ }^{2}$, N. Parietti ${ }^{3}$, S. Rossi ${ }^{3}$, P, Tiraboschi ${ }^{4}$, G.C. Riccitelli ${ }^{3}$ \\ 1 Ente Ospedaliero Cantonale, Bellinzona, Switzerland \\ 2 Center of Excellence for the treatment of Alzheimer's disease, European Biomedical \\ Research Foundation \\ 3 Neurocenter of southern Switzerland, Department of Neurology, Memory Clinic, Neu- \\ ropychology and Behavioral Neurology Research Unit, Switzerland \\ 4 IRCCS Carlo Besta, Milano, Italy
}

Background: Awareness is the capability of an individual to accurately evaluate and report about his/her abilities and limitations [1]. In cross-sectional studies of patients with Alzheimer's Disease (AD), awareness has appeared to decrease with increasing dementia severity, being negligible in mild-disease stages but substantially compromised in advanced disease [2]. In addition, the relationship between awareness domains and behaviouralpsychological symptoms in Alzheimer's disease (AD) remains unclear. In this study was investigate the effects of awareness domains on mild AD patients' emotional-behavioural disturbances and caregivers' stress accounting for demographic and clinical variables.

Material and methods: overall awareness and cognitive, emotional and functional domains were investigated in 60 mild AD patients and 60 related caregivers using the Questionnaire of identification of deficits. The patients' cognitive functioning and psychoaffective/psychiatric symptoms, and their caregivers' stress, were also assessed. Patients were classified as preserved (AD_AP) and impaired (AD_AI) awareness. Hierarchical linear models were applied to explore the effects of awareness domains on psychological and behavioural measures.

Results: unawareness was more frequent for emotional and functional disturbances than for cognitive deficits. AD_AP patients were less engaged in social and leisure activities and had higher rates of psycho-affective disturbances, while AD_AI had higher rates of psychiatric and behavioural disorders. Higher global awareness and higher awareness of 
cognitive alterations respectively explained $32 \%$ and $25 \%$ of the variance for depression (both $p:<0.001$ ), higher awareness of emotional disturbances explained $23 \%$ of the variance for anxiety $(p=0.022)$. Impaired awareness explained $33 \%$ of the variance for apathy symptom $(p<0.001)$. Unawareness was also associated with higher caregivers' stress.

Conclusions: independently of demographic and clinical characteristics, subjects with early-stage of AD and higher level of awareness of cognitive and emotional alterations showed psycho-affective symptoms (depression and anxiety). On the other hand, unawareness of deficits, independently of related domains, appears to explain a portion of apathy. The introduction of accurate evaluation of global awareness and its domains in the clinical assessment could contribute to ameliorate the intervention for care and management of mild AD Patients.

P41 Anxiety, Depression, and Apathy as Predictors of Cognitive Decline in Patients with Parkinson's Disease-A Three-Year Follow-up Study

\author{
K. Toloraia ${ }^{1}$, A. Meyer ${ }^{1}$, S. Beltrani ${ }^{1}$, P. Fuhr ${ }^{1}$, R. Lieb ${ }^{2}$, U. Gschwandtner ${ }^{1}$ \\ 1 University Hospital Basel, Switzerland \\ 2 University of Basel, Switzerland
}

Background: Anxiety is one of the most common neuropsychiatric symptom in Parkinson's disease (PD) patients. It impairs cognitive functioning and has a profound impact on quality of life. This follow-up study aims to investigate the predictive value of anxiety, depression and apathy on the development of Mild Cognitive Impairment (MCI) in PD-patients.

Methods: Twenty-nine cognitively unimpaired PD patients (mean age 68.2 $\mathrm{SD} \pm 7.12$ years; 13 women) participated in this study. At Baseline (BL) levels of apathy (Apathy Evaluation Scale, AES), depression (Beck Depression Inventory, BDI-II), and anxiety (Beck Anxiety Inventory, BAI), were assessed. Cognitive status was reassessed three years later according to $\mathrm{MCI} /$ non-MCI status. For statistics, we used binary logistic regression and receiver operating characteristic curve (ROC) analysis to examine anxiety, apathy, and depression at $\mathrm{BL}$ as a predictor of $\mathrm{MCI}$ status three years later.

Results: Eight of the 29 patients developed MCI. Anxiety level at BL was found to predict MCI status at three-year follow-up $(\mathrm{OR}=1.20, \mathrm{CI}=1.02-1.41, p=0.02)$, while depression $(\mathrm{OR}=1.16, \mathrm{CI}=0.93-1.47, p=0.20)$ and apathy $(\mathrm{OR}=1.06, \mathrm{CI}=0.92-1.23, p=0.40) \mathrm{did}$ not predict MCI status. The area under the ROC curve (AUC) of BAI for discriminating PD-non-MCI from PD-MCI was $0.79(\mathrm{CI}=0.61-0.98)$. The optimal classification threshold yielded a sensitivity of $75.0 \%$ and a specificity of $76.2 \%$. Neither apathy nor depression at BL discriminated between PD-non-MCI patients from PD-MCI three years later.

Conclusions: This study demonstrates that anxiety increases the risk of developing MCI in PD patients, while apathy and depression do not.

P42 Deep Brain Stimulation as an Adjunctive in Drug Resistant Therapy: Analysis of Success Rate and Possible Predictors of Response in a Monocentric Swiss

Patient Cohort
A. Belvedere ${ }^{1}$, R. Renzel ${ }^{2}$, L. Stieglitz ${ }^{3}$, L. Imbach ${ }^{2}$
1 UnsiversitätsSpital Zürich, Klinik für Neurologie, Switzerland
2 Swiss Epilepsy Clinic, Klinik Lengg AG, Switzerland
3 Klinik für Neurochirurgie, Universitätsspital Zürich, Switzerland

Background: Deep brain stimulation deep brain stimulation (DBS) of the anterior nucleus of the thalamus has recently emerged as an adjunctive therapy for patients suffering from drug-resistant focal epilepsies. Yet the reported success rates varies widely in different 
studies (Li M; Cook M; Epilepsia 2018; 59(2): 273-290). In addition, the individual response to this treatment option is hard to predict. Therefore, the question remains which clinical parameters predict a good therapeutic response and accordingly which patients are best suited for this treatment option.

Material and methods: We included 13 patients with drug resistant focal epilepsy in this retrospective monocentric study, who had undergone DBS between 2011 and 2020 at the University Hospital Zurich. Based on a structured follow-up 6 and 12 months after DBS, seizure frequency and seizure types and severity, side-effects of DBS, pharmacotherapy were documented and compared to the situation during the last three months before DBS as baseline condition. We correlated these clinical parameters as well as stimulation parameters with the response to DBS.

Results: DBS resulted in a significant improvement of seizure frequency in seven (54\%) out of 13 patients (ILAE outcome score 1-4), with two of these patients (15\%) even becoming temporarily seizure free. The responder and non-responder-group did not differ significantly with respect to age, sex, seizure type, frequency and medication. However, there was a tendency towards early-onset epilepsies and frontal epilepsies responding less to DBS- treatment. Observed side effects were mostly mood changes (observed in five patients; 38\%) but occurred exclusively under high amplitude DBS (above 3V).

Conclusions: In at least half of the patients with drug-resistant epilepsy, DBS proved to be a successful third-line therapy. Our data suggest that DBS might be less effective in patients with frontal lobe epilepsies and in patients with early-onset epilepsies. Further multicenter trials will be needed to identify reliable predictors of patient response to deep brain stimulation.

P43 Management of Lambert-Eaton Myasthenic Syndrome (LEMS) in the Context of Immunecheckpoint-Inhibitor Treatment-A Clinical Case Report

\section{B. Wagner ${ }^{1}$, A. Beuttler ${ }^{1}$, N. Kiriakidis ${ }^{1}$, M. Jörger ${ }^{1}$, R. Käufeler ${ }^{2}$, T. Hundsberger ${ }^{1}$ \\ 1 Kantonsspital St. Gallen, Switzerland \\ 2 Neurologische Praxis St. Gallen, Switzerland}

Introduction: Lambert-Eaton-Myasthenic Syndrome (LEMS) is considered the most common paraneoplastic disease in neurological patients. With increasing use of anticancer immunotherapy, the prevalence of immunotherapy-related neurological adverse events (irNAE) is rising.

Case Description: A 61-year old male patient presented with muscular weakness in the extremities for two months and difficulties climbing the stairs. He did not recognize a worsening over the course of the day and had no further symptoms.

Initial neurological examination disclosed no pathological findings. Electrodiagnostic studies (EDX) revealed a decrement after repetitive nerve stimulation and an increment after maximal muscle contraction. Electromyography was unremarkable, CK was slightly elevated. Acetylcholine receptor antibodies were normal, anti-voltage gated calcium channel antibodies (VGCC-Ab) were elevated, anti-SOX-1-Ab were positive.

The combination of a myasthenic syndrome, a decrement-increment-pattern and VGCC-Ab led to the diagnosis of a LEMS. Positive SOX-1-Ab were suspicious of paraneoplastic origin. The patient was a current smoker and reported night sweats. A chest-CT-scan revealed prominent FDG-PET positive mediastinal lymph nodes leading to the diagnosis of a limited-stage SCLC. Chemotherapy with cisplatin and etoposide and adjuvant thoracic irradiation was initiated. The patient improved and staging disclosed partial remission. 
After the first cycle of maintenance therapy with the anti-PDL-1 monoclonal antibody $(\mathrm{MAb})$ atezolizumab within a clinical trial, muscular weakness worsened again. Neurological examination showed proximal paraparesis, reflexes showed facilitation despite a decrease of VGCC-Ab as a result of tumor-specific therapy. A symptomatic therapy with 3,4 Aminopyridine was initiated, Atezolizumab was discontinued.

Discussion: We report a patient suffering from paraneoplastic LEMS in the context of a SCLC. Besides tumor-specific therapy being the treatment of choice, immunotherapy using PDL-1-targeting $\mathrm{mAb}$ is increasingly being implemented in different tumor entities. While autoimmune is a well known potential toxicity, data is very limited on PDL-1-targeting $\mathrm{mAb}$ associated exacerbation of paraneoplastic autoimmune phenomena including LEMS. This case adds important information to this emerging field, including an important discussion on management of LEMS.

P44 Patients with Amyotrophic Lateral Sclerosis without Behavioural Disorders Are not Impaired in Recognising Negative Facial Emotions

\section{Gerber ${ }^{1}$, D. Lulé ${ }^{2}$, N. Braun ${ }^{3}$, K. Schweikert ${ }^{4}$, M. Weber $^{3}$, R. Kressig ${ }^{5}$, O. Piguet ${ }^{6}$, M. Sollberger ${ }^{7}$}

1 Memory Clinic, University Department of Geriatric Medicine FELIX PLATTER/Faculty of Psychology, University of Basel, Switzerland

2 Department of Neurology, Ulm University Medical Center, Germany

3 Kantonsspital St. Gallen Muskelzentrum/ALS Clinic, Switzerland

4 ALS Sprechstunde, REHAB Basel/Department of Neurology, University Hospital Basel, Switzerland

5 University Department of Geriatric Medicine FELIX PLATTER, Switzerland

6 School of Psychology and Brain and Mind Centre, The University of Sydney, Australia

7 Memory Clinic, University Department of Geriatric Medicine FELIX PLATTER/Department of Neurology, University Hospital Basel, Switzerland

Background and Objectives: Evidence on the presence of emotion recognition disturbance in amyotrophic lateral sclerosis (ALS) is mixed. Likely explanations for this variability are heterogeneity of clinical symptomatology with regards to cognition, behaviour and affect, as well as heterogeneity of instruments used to assess emotion recognition. Here, we focused on pure ALS patients (i.e., without dementia, and without behavioural and affective disorders). We also focused on negative emotions as recognition of these emotions tend to be more affected than positive emotions. ALS patients were tested with the Facial Emotion Intensity Rating Task-Congruent and Incongruent (FEIRT-CIC), a sensitive and short test of facial emotion recognition task recently developed. We investigated whether patients would experience difficulty in discriminating among the four basic negative facial emotions (i.e., anger, disgust, fear, and sadness).

Materials and Methods: We compared the capacity in negative facial emotion discrimination between 19 ALS patients [13 without and 6 with cognitive deficits according to the Strong et al. (2017) diagnostic criteria] without behavioural and affective disorders and 19 healthy controls, matched for age, sex and education. Scores in the two groups were compared using the Wilcoxon-Mann-Whitney-test and Receiver Operating Characteristic (ROC) analyses.

Results: We found no significant difference in overall negative facial emotion discrimination between the two groups $(p=0.14)$. The ALS group's median score was at 1.17 $($ range $=3.75)$ and the healthy controls' median score was at 1.96 (range $=3.99)$. ALS patients were only impaired at borderline in discriminating anger from the other three emotions ( $p=0.045$ ), showing an area under the curve (AUC) of 0.69 .

Conclusions: ALS patients without dementia, behavioural and/or affective disorders are not impaired in negative facial emotion discrimination. Previous reports of facial emotion 
recognition deficits in ALS patients may have been driven by subgroups of ALS patients with co-existing dementia or psychiatric disturbances. Next, we plan to examine the capacity of negative facial emotion discrimination in ALS patients with behavioural disorders.

P45 Neuromuscular Ultrasound (NMU) as a Decisive Diagnostic Tool Diagnosing Myasthenia Gravis as an Underlying Cause of Respiratory Failure: A Case Report

\title{
N. Kiriakidis, D. Leupold, G. Kägi, G. Kleger, B. Tettenborn
}

Kantonsspital St. Gallen, Switzerland

Background: Respiratory failure is one of the main causes of morbidity and mortality in patients with neuromuscular junction disorder (NMJD) [1]. NMU is an established diagnostic tool for evaluation of diaphragm function [2,3]. We report a case of an elderly patient where diaphragmatic ultrasound was crucial in diagnosing NMJD as an underlying cause of acute respiratory failure.

Case: A 79 y old woman underwent a planned surgery (ablatiomammae due to breast cancer), where she required general anaesthesia with intubation. A few hours after extubation she developed recurring episodes of hypercapnic hypoxaemia with respiratory acidosis and somnolence. Clinically she presented with a lack of chest wall excursions. On clinical examination she had full muscle power and no sensory disturbances were detectable; reflexes were symmetrical present. On history she hasn't reported about breathing difficulties, diplopia or generalized muscle weakness prior to these episodes. She required re-intubation and intermittent non-invasive ventilation (NIV) over time due to recurrent raising paCO2 levels and tiring out.

Diagnostics: Phrenic nerve conduction studies and needle EMG of the diaphragm were not feasible in the ICU setting and post-surgery conditions. However, diaphragmatic ultrasound showed a severe weakness and markedly reduced breath-correlated variability of the diaphragm (thickening ratio). The differential diagnosis of a NMJD was very likely, after we ruled out other causes of neuromuscular respiratory failure, such as bilateral lesions of phrenic nerve or immune-mediated neuropathies. Later in the course elevated Ach-Antibodies supported the diagnosis of MG. A course of intravenous immunoglobulins (IVIg) was established.

Conclusions: Isolated diaphragm dysfunction and acute respiratory failure is an uncommon initial presentation of a NMJD. Phrenic nerve conduction studies and needle EMG of the diaphragm are often technically challenging, especially in the setting of ICU. Therefore, ultrasound imaging is a non-invasive, bedside, accurate and safe technique allowing assessment of diaphragm anatomy and function. In this patient severe weakness and a reduced bilateral breath-correlated variability of the diaphragm, shown on NMU, was a crucial hint leading to the underlying cause of a primary neuromuscular respiratory failure (MG). In patients with acute dyspnoea, unexplained respiratory failure or weaning difficulties on ICU, a NMJD should always be considered.

\section{P46 Volar Wrist Arthrodesis for Treatment of the Spastic Wrist}

\author{
E. Rothenfluh ${ }^{1}$, A. Schiller ${ }^{1}$, C. Erling ${ }^{2}$, I. Besmens ${ }^{1}$, M. Calcagni ${ }^{1}$ \\ 1 Universitätsspital Zürich, Switzerland \\ 2 Manus Praxis, Freiburg, Switzerland
}

Background: Among the deformities in patients with spasticity due to cerebral palsy or strokes the wrist is the major component, which adversely affects hand function and appearance. In mild to moderate deformities, they can be corrected with soft tissue reconstructions such as flexor tendon release, lengthening or transfers. In severe cases the wrist arthrodesis may be an alternative surgical option with previously reported, reliable 
outcomes. In contrast to the classic dorsal approach, the technique described by Gong et al. is based on a volar plate fixation. Concomitant flexor tendon release is possible. Prominent hardware and undesirable scars on the dorsum of the wrist can be avoided. We present the results of 5 cases in 4 patients with regard to degree of correction, satisfaction and complications.

Material and methods: We performed a total wrist arthrodesis with proximal row carpectomy in 4 patients and 5 wrists through a palmar approach. A carpal tunnel release was performed and the superficial flexor tendons were transferred to the deep flexor tendons (superficialis to profundus procedure). This was combined with lengthening or tenotomies of other tendons in individual cases. The outcome was assessed on the basis of the degree of correction on the lateral view of the X-ray films.

Results: We report about the results of 4 patients, all of which were male and 19 and 68 years old (average 35 years). The oldest patient suffered from spastic tetraplegia after ischemic stroke while the others had cerebral palsy. Follow up time was 3 to 42 months (average 19 months). The patient with 3 months follow up was recently operated, but already shows good outcome with no complications. One patient showed screw loosening without need for intervention, another patient had a haematoma postoperatively. This was managed expectantly and had no consequence. Bony fusion was achieved in all cases after 3 months. The preoperative palmar angulation of the wrist was between 80 and 115 degrees on plain X-rays (average 99.4). The postoperative angles were 5 to 50 degrees (average 24 degrees). The correction achieved was 30 and 110 degrees (average 75.6 degrees). All patients and care takers were satisfied.

Conclusions: A volar wrist arthrodesis can be very useful in spastic patients. It allows concomitant soft tissues procedures, such as flexor tendon lengthening for fingers and thumb as well as tenotomies. It avoids prominent hardware and provides cosmetically desirable outcome.

\section{P47 Adult-Onset Distal Spinal Muscular Atrophy (SMA) with Identification of Heterozygous VRK1-Gene Mutation-Case Closed?}

\section{A. Oertli, E.P. Wilder-Smith}

\section{Luzerner Kantonsspital (LUKS), Switzerland}

Background: Spinal Muscular Atrophy (SMA) is characterized by loss of motor neurons and progressive muscle weakness. About $95 \%$ of SMA cases are caused by autosomal recessive mutations in SMN1 (survival of motor neuron 1) gene on chromosome 5 (5q-SMA). Medical treatment options are only available for 5q-SMA. SMA mutations in other genes have been reported (non-5q-SMA). We report a case of adult-onset distal SMA (dSMA) with detection of a heterozygous mutation in VRK1 (vaccinia-related kinase 1) gene.

Case report: A 51-years-old man presented with progressive atrophy of calve muscles and gait instability for 2 years. Hiking and walking in steep terrain was more difficult. He denied sensory symptoms except nocturnal paresthesia's due to carpal tunnel syndrome. He had no dysphagia, dysarthria, visual symptoms or muscle cramps. He was athletically active as a young man in semi-professional wrestling. There were no family members with muscle diseases, gait problems or foot deformities. On the first examination he had atrophy and mild paresis of distal legs with normal reflexes except for absent ankle reflexes and absent patellar reflex left. Four years later there was a progression of distal and additionally proximal leg paresis.

Results: Routine NCS showed normal nerve conductions with normal amplitudes of motor responses and in EMG active and chronic denervation in proximal and distal leg muscles. Brain MRI was normal. Whole Body MRI showed fatty atrophy of proximal muscles and massive fatty atrophy of distal leg muscles. Genetic testing detected a heterozygous mutation in the VRK1 gene (c.707dupT, p.Ala237GlyFS*7) leading to a frameshift and a premature stop codon. 
Conclusions: Mutations in VRK1 gene located on chromosome 14q32 are associated with a wide phenotypic spectrum: spinal muscular atrophy with pontocerebellar hypoplasia, motor and sensory axonal neuropathy plus microcephaly, dSMA and ALS with microcephaly or intellectual disability. Our mutation p.Ala237GlyFS*7 has not been described yet. All reported VRK1-mutation associated with dSMA are homozygous or compound heterozygous. The previously reported mutation p.R358X also with premature stop codon is not disease causing in a heterozygous manner. Most likely there is an undetected mutation in our patient.

\section{P48 Botulinum Toxin Restores Sleep Homeostasis in Cervical Dystonia}

\section{S. Caverzasio, N. Amato, G. Chiaro, C. Staedler, A. Kaelin-Lang, S. Galati}

Neurocenter of Southern Switzerland, Regional Hospital Lugano (EOC), Lugano, Switzerland

Background and Objectives: An alteration of brain plasticity has been proposed to play a key role in the pathophysiology of cervical dystonia (CD). Several lines of evidence support the hypothesis that sleep regulates brain plasticity. We aimed to test the hypothesis that an alteration of the sleep homeostasis mechanism may be involved in the pathogenesis of $\mathrm{CD}$, by exploring sleep before and after the clinical effective treatment with botulinum toxin (BoNT).

Methods: 10 CD patients and 8 control subjects (CTL) underwent two video-polysomnography with high-density electroencephalography (vPSG-hdEEG), one week before (Time, TpreBoNT) and two weeks after (Time, Tpost-BoNT) the BoNT treatment. NREM sleep was extracted, combined and subdivided into 2 equal parts (early and late sleep respectively). The slow wave activity (SWA) power was then calculated and compared between and within groups.

Results: CTL and CD at Tpost-BoNT showed a significant SWA power decrease throughout the night. That was not the case in CD at Tpre-BoNT. In the early sleep, CD patients showed at Tpost-BNT a frontal increase and parietal decrease in SWA power compared to CD at Tpre-BoNT.

Conclusions: Our data highlighted a pathophysiological relationship between sleep and $\mathrm{CD}$ and provided novel insight into the transiently central plastic effect of BoNT. Further studies are needed to clarify this relationship, as well as the role of BoNT as a temporary SWA-enhancing therapy in CD patients.

P49 Does Quantitative Electroencephalography Refine Preoperative Cognitive Assessment in Parkinson's Disease Patients Treated with Deep Brain Stimulation? A Follow-up Study

\section{Saleh, A. Meyer, M. Chaturvedi, S. Beltrani, U. Gschwandtner, P. Fuhr}

University Hospital Basel, Switzerland

Objective: Deep brain stimulation (DBS) in Parkinson's disease (PD) is associated with an increased risk of postoperative cognitive deterioration. Preoperative neuropsychological testing can be affected and limited by the patient's collaboration in advanced disease. The purpose of this study was to determine whether preoperative quantitative electroencephalography (qEEG) may be a useful complementary examination technique during preoperative assessment to predict cognitive changes in PD patients treated with DBS.

Methods: We compared the cognitive performance of 16 PD patients who underwent bilateral subthalamic nucleus DBS to the performance of 15 PD controls (matched for age, sex and education) at baseline and at 24 months. Cognitive scores were calculated for all patients across 5 domains. A preoperative 256-channel resting EEG was recorded from each patient. We computed the global relative power spectra. Correlation and linear regression 
models were used to assess associations of preoperative EEG measures with postoperative cognitive scores.

Results: Slow waves (relative delta and theta band power) were negatively correlated with postoperative cognitive performance while faster waves (alpha 1) was strongly positively correlated with the same scores (overall cognitive score, attention, executive function). Linear models revealed an association of delta power with the overall cognitive score ( $p=0.00409$, adjusted R-squared 0.6341). Verbal fluency showed a significant decline after DBS surgery which was correlated with qEEG measures.

Conclusions: To analyse the side effects after DBS in PD patients the most important parameter is verbal fluency capacity. In addition, correlation with EEG frequency bands might be useful to detect particularly vulnerable patients for cognitive impairment and be supportive in the selection process of patients considered for DBS.

\section{P50 Reduced Alpha Suppression \& Altered EEG Signal Complexity in Parkinson's Disease-Signatures of Cognitive Impairment?}

\section{S. Keller, A. Meyer, U. Gschwandtner, S. Beltrani, K. Toloraia, P. Fuhr}

University Hospital Basel, Switzerland

Objective: In a cross-sectional setting both alpha suppression and complexity of alphaband oscillations are evaluated as diagnostic biomarker for assessing MCI in early stages of PD.

Background: In a healthy human brain alpha-band oscillatory activity $(8-13 \mathrm{~Hz})$ is present during eyes closed (EC) condition but is suppressed when eyes are open (EO). Alpha oscillations have an important role in attentional processes. In PD, cognitive decline may be accompanied by pathological levels of attention. Therefore the state of mild cognitive impairment (MCI) in PD, which is a transitional state between normal cognition (PD-NC) and overt dementia (PD-D), is hypothesized to exhibit abnormal behaviour compared to healthy controls or PD-NC patients.

Methods: 24 healthy controls (HC, 9 females, medians: age 66.5 yrs, edu. 14 yrs) and 31 PD-NC individuals (14 females, medians: age 69.0 yrs., edu. 14 yrs, UPDRS 15, LED 490.0, disease duration 32 mos) were compared with 8 individuals diagnosed with PD-MCI matched for age, sex and education (3 females, medians: age 65.5 yrs, edu 16 yrs, UPDRS 15.5, LED 784.5, disease duration 50 mos). High-density EEG recordings were obtained during eyes open (EO) and eyes closed (EC) condition. Signal complexity, quantified based on Tsallis entropy (TE) [1], as well as relative band power (rBP) were evaluated for the alpha band. The resulting signal complexity and band power values were then compared across groups where grouping was based on an individuals' diagnosis.

Results: Evaluation of rBP during EC and EO condition showed significant or close to significant intra-group differences for HC $(p=0.00001)$ and PD-NC $(p<0.0501)$ but not for PD-MCI $(p>0.5)$. Alpha band signal complexity is increased in EO condition across all groups compared to EC. While the PD-MCI group exhibited a non-significant increase $(p>0.9), \mathrm{HC}$ and PD-NC showed significant differences (HC: $p=0.0001$ and PD-NC: $p=0.003)$.

Conclusions: In conclusion, mild cognitive impairment in PD may manifest in the alpha band (8-13 Hz) through impaired ability to perform alpha suppression and through increasingly similar levels of signal complexity between EC and EO condition. The results apply to groups, and may help to define study populations for clinical trials, but cannot be applied in their present form for individual treatment decisions or counseling. 
P51 The Basel Version of the Awareness of Social Inference Test-Theory of Mind (BASIT-ToM): Preliminary Validation Analyses in Healthy Subjects

\author{
M. Jarsch ${ }^{1}$, O. Piguet ${ }^{2}$, C. Sluka ${ }^{3}$, N. Huber ${ }^{4}$, R.W. Kressig ${ }^{5}$, A.U. Monsch ${ }^{4}$, M. Berres ${ }^{6}$, \\ M. Sollberger ${ }^{7}$
}

1 Memory Clinic, University Department of Geriatric Medicine FELIX PLATTER/Faculty of Psychology, University of Basel, Switzerland

2 School of Psychology and Brain and Mind Centre, The University of Sydney, Australia

3 University of Basel and University Hospital Basel, Switzerland

4 Memory Clinic, University Department of Geriatric Medicine FELIX PLATTER

5 University Department of Geriatric Medicine FELIX PLATTER, Switzerland

6 Faculty of Mathematics and Technology, University of Applied Science Koblenz, Germany

7 Memory Clinic, University Department of Geriatric Medicine FELIX PLATTER/Department of Neurology, University Hospital Basel, Switzerland

Background and Objectives: Impairment of Theory of Mind (ToM) abilities occurs in a wide range of brain disorders. Therefore, its reliable and ecologically valid examination is a crucial part of any comprehensive neuropsychological assessment. In this regard, an established English-language test identifying deficits in ToM abilities is the "The Awareness of Social Inference Test-Social Inference Minimal" (TASIT-SIM). However, a comparable German-language ToM test is currently missing. In this study, we aimed to develop an adaption of the TASIT-SIM in German language for use in clinical populations.

Materials and Methods: We selected 10 scenes [three scenes per message type (i.e., honesty, simple sarcasm, paradoxical sarcasm) and one practice scene] out of the 30 TASIT-SIM scenes. In collaboration with a film institute and professional actors, we filmed each scene at three different intensities. Then, we administered these intensity version scenes to 240 cognitively and mentally healthy participants, equally distributed in sex and age, ranging from 35 to 92 years.

Results: By applying Rasch analysis, the one intensity version per scene was selected that showed in general neither floor nor ceiling effects in the majority of the ToM questions in participants whose ToM abilities were in the medium range.

Conclusions: We have developed the first German-language TASIT-SIM adaption, i.e., the "Basel Version of the Awareness of Social Inference Test - Theory of Mind" (BASIT-ToM). The BASIT-ToM comprises the strengths of the TASIT-SIM, while it overcomes its limitations such as inconsistencies in cinematic realisation, unbalanced proportion of different types of mentalisation and ceiling effects. Next, the BASIT-ToM needs to be validated in clinical populations with neuropsychological disorders.

\title{
P52 iPhone Exophthalmometry: A Novel Approach to Measure Eyeball Protrusion
}

\author{
T. Popov ${ }^{1}$, F. Fierz ${ }^{1}$, C. Bockisch ${ }^{2}$, K. Weber ${ }^{3}$ \\ 1 Universitätsspital Zürich, Switzerland \\ 2 Universitätsspital Zürich, Neurology/Ophthalmology/ENT, Switzerland \\ 3 Universitätsspital Zürich, Neurology \& Ophthalmology, Switzerland
}

Introduction: Accurate and reproducible measures of abnormal eyeball protrusion are important for the diagnosis of exophthalmos, as well as following patients with Grave's orbitopathy and retroorbital masses like meningioma. The current clinical gold standard for measuring abnormal eyeball protrusion is the Hertel exophthalmometer, which is prone to reading errors and rather inconvenient to use. Our objective is to develop and validate an accurate and easy to use smartphone application to measure exophthalmos in patients. 
Methods: 20 healthy volunteers and 20 patients with exophthalmos of different etiology were examined twice within an interval of minimum 2 weeks or after exophthalmoschanging treatment by 3 operators using an iPhone11 TrueDepth camera, a high-resolution 3D-face-scanner (Artec Space Spider), and a Hertel-exophthalmometer as reference. The obtained 3D-images from the scanners were further processed and analyzed using a custom exophthalmos analysis algorithm to determine the distance between the pupillary plane and the lateral orbital rim. The results were then compared to the measurements obtained with the Hertel exophthalmometer.

Results: Preliminary data shows good accuracy and precision of the eyeball protrusion measurements using the 3D scanners on healthy individuals and patients, compared to the Hertel exophthalmometer, with interclass correlation coefficients (ICC) of 0.965 for the high-resolution scanner and 0.963 for the smartphone. We observed a high inter-rater agreement for both the high-resolution scanner (ICC of 0.998) and the smartphone (ICC of 0.983 ) as well as high test-retest reliability (ICC for Artec 0.967 and ICC for iPhone 0.935). Conclusions: The results suggest high precision and accuracy, as well as a high interoperator reliability and test-retest reliability for the novel scanning methods. Smartphone exophthalmometry makes measurement and follow-up of eyeball protrusion quick, easy and objective. Our mobile medical application holds promising potential to replace the traditional Hertel exophthalmometer in the future. Further optimization is underway to make it ready for the ophthalmologist's pocket.

P53 Beyond Conventional MRI: Improving Sensitivity in mTBI Imaging of Individual Patients-A Case-Control-Study

\author{
S. Ulmer ${ }^{1}$, A. Palla ${ }^{2}$, C. Fuchs ${ }^{3}$, N. Simard ${ }^{4}$, M.D. Noseworthy ${ }^{5}$ \\ 1 neurorad.ch, Neuroradiology, Zurich; Switzerland \\ 2 Swiss Concussion Center; Switezrland \\ 3 SportClinic Zürich, Switzerland \\ 4 TBI Finder, Hamilton, Canada \\ 5 McMaster University, Hamilton, Canada
}

Gold standard to depict posttraumatic brain change is susceptibility weighted MR imaging (SWI) at 3T. However, SWI only detects residual blood products and is insensitive to microstructural or physiologic damage i.e., caused by blunt head trauma - despite persistent patient symptoms. We report on a new way to identify post-traumatic brain lesions using MRI resting state functional MRI (fMRI) and diffusion tensor imaging (DTI). Both scans are mainly used for neuronavigation in brain tumor surgery and are routinely available on most MRI systems. For over 2 decades now, DTI has also been used in brain trauma imaging in professional athletes of collision sports.

Our patient is a 16 yo junior national ice hockey player with a history of recurrent blunt head traumas caused by various "bodychecks", $3 \times$ with loss of consciousness. Following the most recent "bodycheck" in February 2021 he complains about headaches, attention disorder, fatigue, visual problems in reading, slight right leg weakness, and gait disturbances. Conventional 3T MRI including SWI is normal.

MRI was performed using a 3T Ingenia (Philips, The Netherlands) using a 15-channel head coil including resting state $\mathrm{fMRI}(\mathrm{TR} / \mathrm{TE}=2000 / 35 \mathrm{~ms}, 3 \times 3 \times 3 \mathrm{~mm})$ and DTI (60-direction; $\mathrm{b}=1000 \mathrm{~s} / \mathrm{mm}^{2} ; \mathrm{TR} / \mathrm{TE}=10,000 / 87 \mathrm{~ms}, 3 \times 3 \times 3 \mathrm{~mm}$ ). TBIFinderTM software was used to calculate a temporal complexity metric (from rs-fMRI) and diffusion tensor metrics (FA, RD, AD, MD) for each brain region, based on the MNI-standard space. The software provides personalized analysis (age/sex matched) through a regional Z-score based on a database of almost 15,000 healthy brains. A standard deviation (SD) of $>2.5$ from healthy controls was considered clinically relevant. 
The most striking changes were found in the right visual cortex (V5, >5 SD) followed by the right inferior parietal lobule (>3.5 SD) in rs-fMRI. Infratentorial the right Crus I was 2.9 SD off healthy controls. Most prominent findings in DTI ( $>3 \mathrm{SD}$ ) were located in the left optic radiation, followed by the right optic radiation $(>2.5 \mathrm{SD})$. Changes of $>2.5 \mathrm{SD}$ were depicted in the left cortico-spinal tract, left uncinate fascicle and left superior longitudinal fascicle. Infratentorial there were changes of 2SD from normal in the right lobule V, VI and the left lobule VI.

All symptoms could be explained by our imaging findings. Conventional MRI at $3 \mathrm{~T}$ is not sensitive enough to depict damage to the brain, thus this advanced imaging protocol should be an add-on for inconclusive MRI.

P54 Real-World Effectiveness Data of Erenumab-Treated Migraine Patients in Switzerland: The SQUARE Study

\author{
E. Schäfer ${ }^{1}$, M.E. Arzt ${ }^{1}$, I. Meyer ${ }^{1}$, A.R. Gantenbein ${ }^{2}$ \\ 1 Novartis Pharma Schweiz AG, Switzerland \\ 2 RehaClinic Bad Zurzach, Switzerland
}

Objective: To present real-world effectiveness data of erenumab from the SQUARE (Swiss QUality of life and healthcare impact Assessment in a Real-world Erenumab treated migraine population) study.

Methods: SQUARE is a non-interventional study which provides clinical effectiveness data on erenumab in a post-marketing setting from both migraine care specialist centers and general neurologists in Switzerland. Migraine patients receiving Aimovig®in accordance with the Swiss label were included if willing and able to participate, with the exception of patients with prior use of any medications targeting the calcitonin gene-related peptide (CGRP) pathway or recent use of investigational drugs. Patients were observed over a period of 24 months.

Results: A total of 173 adult patients were included in 19 centers. Here, we will present real-world effectiveness data of erenumab at month 6 compared to baseline, including scores of Headache Impact Test (HIT-6), modified (monthly) migraine disability assessment test (mMIDAS), and impact of migraine on partners and adolescent children (IMPAC).

Conclusions: These are among the first prospectively collected data on erenumab under routine medical care. Results from the SQUARE study will provide insights into the effectiveness of a CGRP receptor-based therapy in a real-world scenario.

P55 Effect of Transcranial Direct Current Stimulation in a Patient with Frontal Chronic Post-Traumatic Encephalopathy

\author{
A. Dontschev ${ }^{1}$, L. Rochat ${ }^{2}$, M. Mouthon ${ }^{1}$, J. Annoni ${ }^{1}$, F. Ribordy Lambert ${ }^{1}$ \\ 1 UNI FRIBOURG, Switzerland \\ 2 HFR—Hôpital Fribourgeois, Switzerland
}

Background \& Objectives: Transcranial direct current stimulation (tDCS) is a potentially effective non-invasive neuromodulation method, used in experimental neurorehabilitation settings to increase neurological and functional recovery $(1,2)$. Interestingly, its indication as a therapy for the rehabilitation of traumatic brain injury is still discussed. Here we proposed to (i) examine the feasibility and adherence to a longer training protocol than previously described in the literature $(2 \times 3$ weeks) and (ii) assess the effectiveness in comparing cognitive training with sham tDCS and cognitive training with $\mathrm{tDCS}$, in a single-case quasi-experimental study involving a patient with chronic working memory (WM) and executive function impairment following a severe traumatic brain injury. 
Material and methods: The participant was a partially institutionalized 46-year-old female (MoCA score 18/30) who sustained a very severe traumatic brain injury at the age of 14 , with residual amnestic and frontal symptoms. WM (2-Back task) and inhibition (Go/No-Go task) were trained following an A-B design, involving first three weeks of cognitive training + sham-tDCS followed by three weeks of cognitive training + tDCS. Neuropsychological visits and fMRI assessments were performed before and after the $\mathrm{A}$ and $\mathrm{B}$ training phases. Results: (i) The participant attended $100 \%$ of the planned appointments and reported overall satisfaction toward her participation; (ii) Several classical indices of performances were extracted from trials of A and B phases and were analysed with Nonoverlap of All Pairs (NAP) (3) and Tau-U (4) index. We identified an overall increase in performance in the $\mathrm{tDCS}$ training phase as compared to sham tDCS training, for the 2-Back task (mediumsized decrease in false alarm rate, Tau-U $=0.8$; large-sized increase in correct rejection rate, $\mathrm{NAP}=0.93)$. In the Go/No-Go tasks however, while the mean response time for Hits was faster (NAP $=0.64)$, accuracy decreased (NAP for all correct responses $=0.43$ ). fMRI data reveal higher right anterior cingulate and medial frontal activities after tDCS than to sham training, for the 2-Back task.

Conclusions: Our study showed that a $2 \times 3$ weeks training protocol was possible and enjoyable for our patient, and that cognitive training coupled with tDCS induced specific performance improvements in WM. These clinical results bear potential for the rehabilitation of executive disorders in patient with severe traumatic brain injury.

\section{P56 Anosognosia for Memory Dysfunction as a Key Determinant of Cognitive and Psychiatric Symptoms in Long COVID}

\section{P. Voruz 1, G. Allali 2 , L. Benzakour ${ }^{2}$, A. Nuber-Champier 1, M. Thomasson ${ }^{1}$, I. Jacot de Alcantara ${ }^{1}$, A. Cionca ${ }^{1}$, P. Lalive d'Epinay ${ }^{2}$, K. Lövblad ${ }^{2}$, M. Nehme ${ }^{2}$, O. Braillard ${ }^{2}$, M. Coen ${ }^{2}$, J. Serratrice ${ }^{2}$, J. Reny ${ }^{2}$, J. Pugin ${ }^{2}$, I. Guessous ${ }^{2}$, B Landis ${ }^{2}$, F. Assal ${ }^{2}$, J. Péron ${ }^{1}$}

1 Clinical and Experimental Neuropsychology Laboratory, Faculty of Psychology, Geneva, Switzerland 2 Hôpitaux Universitaire de Genève HUG, Switzerland

Based on previous observation on 45 patients (1), the aim of this study on 102 patients at 6-9 months post-SARS-CoV-2 infection was to determine whether the separation of patients according to anosognosia for memory dysfunction (AMD) was statistically discriminant for cognitive functions, psychiatric symptoms, olfactory performance, perceived fatigue and quality of life. Secondly, our study aimed to determine whether anosognosic patients (AP) had a distinct cognitive-psychiatric profile of long COVID from nosognosic patients (NP). For this study 102 patients (mild: $n=45$; moderate: $n=34$; severe: $n=23$ ) at $227.07 \pm 42.69$ days after infection with SARS-CoV-2 completed a battery of neuropsychological, psychiatric, fatigue, quality of life, olfactory recognition, dyspnea tests and questionnaires. Patients were divided according to their score of AMD regardless of the severity of the respiratory symptomatology in the acute phase (anosognosic: $n=26$; nosognosic: $n=76$ ) by calculating a self-appraisal discrepancy (SAD) (2). Groups were matched for age, sociocultural level, handedness, gender and clinical variables.

The present results suggest, that AMD is a discriminant factor that enable to predict the presence of (i) a greater impairment of memory and executive functions, (ii) less selfreported psychiatric symptoms, and (iii) a greater self-reported quality of life. Moreover, AP were unconscious of their olfactive dysfunctions.

To our knowledge, our results indicate for the first time that a specific behavioral variable, in this case AMD, could be a discriminating factor and marker of neurological impairment following SARS-CoV-2 1. Interestingly, our results in AP long COVID patients are very similar to previous findings for anosognosic patients in $\mathrm{MCI}$ and Alzheimer's disease with a higher proportion of memory and executive disorders, as well as fewer psychiatric symp- 
toms and a better quality of life than NP (3). On the basis of these results, two hypotheses could be proposed to explain the presence of this particular neuropsychological phenotype. First, SARS-CoV-2 damages the central nervous system (particularly limbic system) and induces these symptoms. Second, it could indicate the presence of a neurological vulnerability in some patients preceding the SARS-CoV-2 infection and the infection could have a triggering effect of an accelerated neurodegenerative process.

\section{P57 Ocular Lateral Deviation-A Clinical Sign Pointing to Lateral Medullary Lesions}

\section{Spiegelberg, A. Tarnutzer}

Kantonsspital Baden AG, Switzerland

Background and Aims: About 9\% of all cerebrovascular events are missed at the initial presentation to the emergency department, with risk of misdiagnosis being much higher in cases with non-specific findings (e.g., dizziness) compared to cases with motor symptoms. Possible reasons for a missed diagnosis of stroke as underlying cause of an acute vestibular syndrome include an incomplete clinical examination and overreliance on CT-based neuroimaging. This underlines the importance of a targeted neuro-otological bedside examination (as e.g., the "HINTS plus" algorithm) in such patients. However, these bedside tests may sometimes be difficult to perform and interpret. Testing for complete ocular lateral deviation (OLD) may be a valuable additional approach, as it can be readily and reliably interpreted by ED physicians or neurologists in charge. Thus, by use of two illustrative patients with an acute lateral medullary stroke we thought to demonstrate how to perform this test and what findings to expect.

Material and methods: Two male patients (aged 54 and 75 years) presenting with newonset dizziness, gait imbalance, dysphagia and nausea were included in this illustrative case series. In addition to targeted bedside neuro-otological testing (including HINTS plus) and MR-imaging including DWI-sequences, patients were clinically assessed for complete OLD after brief (3-5 s) eyelid closure. Clinical findings were then correlated with lesions identified on MR-imaging.

Results: In both patients with MR-confirmed acute lateral medullary strokes complete ipsilesional OLD after brief eyelid closure was found at the initial presentation. After reopening their eyes, they corrected for OLD by a series of hypometric centripetal saccades. Conclusions: Complete ipsilesional OLD after brief ( $3-5$ s) eyelid closure is highly specific for a central (usually ischemic medullary) lesion as demonstrated in this case series. In a recent publication (1) a specificity of $100 \%$ for detecting stroke in patients with acute vestibular syndrome (AVS) was reported along with a sensitivity of OLD for a central cause of $12 \%(12 / 100)$. Thus, testing for OLD is recommended in patients with acute-onset persistent vertigo, meeting diagnostic criteria for acute vestibular syndrome. Noteworthy, radiologic OLD is usually seen in the context of continuous eyelid closure for several minutes (2) and therefore does not allow a distinction between central and peripheral causes.

P58 Brain Structures of Early Diglossic Bilinguals: Impact of German/Swiss German Bilingualism on Grey Matter Density of Language Control Areas in University Students

\section{Berger ${ }^{1}$, M. Mouthon ${ }^{2}$, L. Jost ${ }^{3}$, J. Annoni ${ }^{4}$}

1 Faculty of Science and Medicine of the University of Fribourg, Switzerland

2 Faculty of Science and Medicine-Neurology Unit of the University of Fribourg, Switzerland

3 Laboratory for Cognitive and Neurological Science (LCNS) of the University of Fribourg, Switzerland

4 Faculty of Science University of Fribourg- Neurology unit, Fribourg Hospital, Switzerland 
Background: In Switzerland, Swiss German (CH-GER) is an oral maternal language while "classic" German is the first written language learned at school. Importantly, children are already exposed to oral classic German in kindergarten, i.e., when they are younger than 7 years of age. There is some evidence that CH-GER can be considered as an autonomous language. This is why Swiss German speaking people are often referred to as diglossic, which is highly related to bilingual. As bilingualism shapes the brain, and particularly language control areas, such regions could be different between populations who grew up with one language compared to those with a diglossic language background.

Aim: This study examines whether bilinguals with a diglossic background and participants with a purely monolingual background until the age of 7 would show differences in grey matter density and volume in language specific and cognitive control regions.

Methods: Brain MRI was analyzed in 11 CH-GER and $18 \mathrm{CH}-F R$ (French) university students in Fribourg, Switzerland. Subjects replied to several questions about their language skills, daily exposure to and age of acquisition of their second language (L2). Subsequently, the T1-weighted anatomical scans of subjects were processed using Statistical Parametric Mapping SPM12 and CAT12 toolbox. Voxel- and Surface-Based-Morphometry (VBM and SBM) were analyzed. We compared the volume and cortical thickness means inside of the region of interest (ROI) the left inferior frontal gyrus (IFG), the left dorsolateral prefrontal cortex (DLPFC), bilateral anterior cingulate cortex (ACC), and caudate nucleus $(\mathrm{CN})$, between the two groups with first language $\mathrm{CH}-\mathrm{GER}$ and $\mathrm{CH}-\mathrm{FR}$. An ANCOVA was performed to exclude confounds (gender, age and total intracranial volume [only for VBM]) and a Bonferroni correction were applied to correctfor the multiple comparison problem (significant threshold $p=0.05 / 4=0.01$ ).

Results: L1-FR group disclosed a higher volume (V) and cortical thickness (CTh) for the left DLPFC (V: $p=0.003$ and CTh: $p<0.001^{*}$ ) as well as for the left IFG (V: $p=0.01$ and CTh: $\left.p<0.001^{*}\right)$. No statistically significant difference was found in ACC and CN $(p>0.01)$.

Conclusions: Some language control areas, the IFG and DLPFC, exhibited a thinner cortical thickness in the $\mathrm{CH}$-GER than $\mathrm{CH}-\mathrm{FR}$ group, suggesting that early diglossic experience may lead to reduced language control areas, possibly through less cognitively costly mechanisms.

\section{P59 Case report: Response of Corticotroph Adenoma to Temozolomide in a Patient with Nelson Syndrome}

\section{Baldauf, W. Jochum, A. Lauber, T. Hundsberger, M. Brändle}

\section{KSSG Kantonsspital St. Gallen, Switzerland}

Nelson syndrome is a rare complication after total bilateral adrenalectomy (TBA), which is a treatment option in controlling hypercortisolism in Cushing's disease refractory to transnasosphenoidal surgery (TNS). Classical findings are growth of an, sometimes pre-existing, ACTH-producing pituitary adenoma, high ACTH secretion and hyperpigmentation. These corticotroph tumors are rare and only limited data regarding treatment exist. We here describe a patient suffering from progressive corticotroph tumor with very good response to Temozolomide, an oral alkylating agent approved for the treatment of glioblastoma (GB). We describe a patient who developed Cushing's disease due to an ACTH-secreting microadenoma of the pituitary gland at the age of 23 years. After TNS, he experienced several relapses over the following 6 years, making two further TNS as well as gamma-knife treatment necessary. Then he turned again symptomatic with overt hypercortisolism, but without MR-tomographical detection of a tumor-relapse. TBA was performed. 10 months later, he developed Nelson syndrome, including recurrence of the corticotroph tumor. The tumor was resected 4 months afterwards. The residual tumor progressed, leading to another gamma-knife treatment, three further TNS, pasireotid, cabergoline and 177LuDOTATOC treatment. Nevertheless, the tumor progressed. Palliative chemotherapy with temozolomide was induced, despite an unmethylated promotor of the methyl-guanine- 
methyltransferase gene (MGMTp-methylation status 3\%) as a negative prognostic and predictive biomarker for treatment response in GB. However, with this therapy, the tumor regressed almost completely. Therapy was terminated after 6 months/cycles. 14 months later, the tumor asymptomatically progressed, unfortunately. Re-Exposure to Temozolomide is ongoing.

Optimal treatment strategy for large Nelson syndrome tumors still needs to be defined. Temozolomide is considered as a last treatment option in favor of local treatment strategies (radiotherapy, TNS). Our case may challenge this concept as temozolomide induced longterm remission without major side effects. Furthermore, temozolomide treatment should also be evaluated despite an unmethylated MGMTp-status as it may not reflect resistance to alkylating chemotherapy like in GB. The 14 months long-term remission is very promising, with few adverse effects of temozolomide. However, the benefit of temozolomide rechallenge in this patient is unclear at this point in time.

\title{
P60 Long-Term Regional Quantification of Microstructural and Functional Abnormalities in Retired Canadian Football League Players
}

\author{
E. Danielli ${ }^{1}$, N. Simard ${ }^{2}$, M. Doughty ${ }^{1}$, S. Ulmer ${ }^{3}$, D.A. Kumbhare ${ }^{4}$, M.D. Noseworthy ${ }^{1}$ \\ 1 McMaster University, Hamilton, Canada \\ 2 TBIFinder inc., Hamilton, Canada \\ 3 neurorad.ch, Zurich, Switzerland \\ 4 University of Toronto, Canada
}

Background \& Goals: Concussions and sub-concussive impacts can cause lasting diffuse axonal injuries [1] and decreased functional activation, leading to dementia [2]. The purpose of this study was to identify and quantify the severity of abnormal structural and functional brain regions in a retired athlete sample. It was hypothesized that longer fibre tracts, more susceptible to tearing, and cortical regions would be more seriously damaged. Materials \& Methods: Retired Canadian Football League (rCFL) players ( $n=17$, all male, aged $58.3 \pm 6.2$ ) were scanned using a 3T MRI system and 32-channel head and neck coil. High-resolution T1-weighted, 60-direction diffusion tensor imaging (DTI), and resting state functional MRI (rsfMRI) scans were acquired for each subject. Age and sex matched control data $(n=88)$ was downloaded from the Alzheimer's Disease Neuroimaging Initiative (ADNI) [3]. MRI data was processed using TBIFinder software to produce personalized regional Z-scores [4,5]. Forty-eight grey and 19 white matter brain regions-of-interest (ROIs) were segmented [6]. The ROI masks were individually multiplied over all rCFL and control subjects to calculate regional DTI (FA, MD, AD and RD) and rsfMRI temporal complexity values (Hurst index, H). A subject-specific total injury burden (IB) was calculated for each metric as mild (1), moderate (2), or severe (3) based on Z-scores that fell 2, 3 or 4 standard deviations from the control means, respectively. Correlations were performed in RStudio for the IB of each metric, with age, head impact (based on position), and career length as covariates.

Results: From the DTI data, the left cingulum had the most outliers: 4 subjects had an FA IB of 1 and one subject had an FA IB of 3. The rsfMRI global complexity data was highly variable between subjects. Four subjects had high H IB scores $(9,11,15,28)$ and four had moderate H IB $(1,1,2,6)$. The highest H IB ROI were the right premotor cortex BA6 $(n=4)$, visual cortex V1 BA17 $(n=3)$, visual cortex V2 BA18 $(n=3)$, and hippocampus dentate gyrus $(n=3)$. Neither FA nor global $\mathrm{H}$ were correlated with age, position or career length. Conclusions: Although there are normal age-related decreases in grey and white matter [7], our study identified decreases in rCFL subjects surpassing that found in normal aging brains. Only one subject expressed a high FA and H IB (FA IB = 3, H IB = 15). Continued research is being conducted to explore relationships between MRI IB and neuropsychological testing. 
P61 Migraine Diagnosis Made in Time Could Prevent Emergency Room Visits-A Prospective Observational Study

\author{
H. Drangova ${ }^{1}$, N. Kofmel ${ }^{2}$, M. Branca ${ }^{3}$, D. Gloor ${ }^{4}$, B. Lehmann ${ }^{2}$, A. Exadaktylos ${ }^{2}$, \\ S. Jung ${ }^{1}$, U. Fischer ${ }^{1}$, C.J. Schankin ${ }^{1}$ \\ 1 Department of Neurology, Inselspital, Bern University Hospital, Switzerland \\ 2 Department of Emergency Medicine, Inselspital, Bern University Hospital, Switzerland \\ 3 CTU Bern, University of Bern, Switzerland \\ 4 University of Bern, Switzerland
}

Introduction: Headache is a common cause for a presentation at the emergency room (ER). Patients are looking for effective treatment, and severe underlying disease has to be excluded. Since migraine is the most common aetiology of headache in the ER, and successful pre-clinical acute treatment might eventually prevent an ER consultation, if the diagnosis of migraine had been given earlier and triptans were already prescribed. The aim of this study is to quantify the situation of missed diagnosis of migraine prior to ER visits. Methods: The presentation for acute headache at the ER was inclusion criterion for this single-centre prospective study. The treating physician assessed if patients had previous headache attacks fulfilling the ICHD-III criteria of migraine, and if they had the diagnosis of migraine prior to the ER visit. Data was correlated with the discharge diagnosis.

Results: 270 patients were included of which $124(46 \%)$ received the diagnosis of migraine at discharge. Of those, 25 never had migraine attacks before, and 52 already had the diagnosis of migraine. 47 (i.e., $38 \%$ of 124) previously had fulfilled the criteria for migraine but had not been given the diagnosis.

Conclusions: More than one third of patients presenting at the ED with migraine attacks could have been given the diagnosis of migraine earlier. Potentially, specific acute treatment might have prevented the presentation at the ED. This study demonstrates the need for better recognition of migraine by pre-hospital healthcare providers including pharmacists, primary care physicians, and neurologists.

Funding: Not applicable.

Conflicts of Interest: All abstracts and information are published as submitted by the authors and are not the responsibility of the CTN journal (Clinical and Translational Neuroscience) or the meeting organizer. 